\title{
Bending Blindly: Exploring the learnability and usability of bend gestures for the visually impaired
}

\author{
by \\ Matthew Ernst
}

A thesis submitted to the Faculty of Graduate and Postdoctoral Affairs in partial fulfillment of the requirements for the degree of

\author{
Masters of Applied Sciences \\ in \\ Human Computer Interaction
}

Carleton University

Ottawa, Ontario

(C) 2015, Matthew Ernst 


\begin{abstract}
This thesis explored the application of bend gestures as a primary method of interaction for the visually impaired and the use of haptic stimulus as non-visual affordances to assist in the teaching and prompting of bend gestures completions.

The first study explored this use of haptic stimulus and presented 48 different tactons to participants who were asked to select one of three bend gesture locations (top left, center and right) and two directions (up or down) they felt it best represented. The second study explored the interaction experience of bend gestures in a visually impaired environment. Participants completed 9 tasks using both bend and touch gestures recording task completion time and participant preference.

We identify results from both studies that can help shape future research in this area of accessibility and potentially increase the overall interaction experience for screen reader based smartphones. The promising results found in study 1 around the pairing of the motor combination and intensity parameters in tacton design, in conjunction with preference for certain bend gestures as a form of input, lays the foundation for future work in this area.
\end{abstract}




\section{Acknowledgements}

First, I would like to thank my advisor Dr. Audrey Girouard for her support and guidance as I tried to balance a demanding work and school schedule. I appreciate her willingness to accept my last minute transfer and ensured I stayed on track balancing school and work to meet an aggressive timeline. Her ability to understand my fragmented thoughts and at times unintelligible verbal communication, and bring logic and structure to my organic process, was greatly appreciated.

I also thank Paul Wright, Alex Eady and Jessica Lo for help during prototype development and providing guidance on fabrication techniques, PHP scripting and hardware problem solving. I also appreciate the support from my boss: Joel Grenier at You.i and his willingness to allow for a flexible work schedule during this whole process and accommodating meetings and an aggressive testing schedule.

Last but not least, I would like to thank my life mate: Susan Maroun who has unequivocally supported me through all stages of this journey. She has allowed me to follow this wild dream and supported me financially and emotionally through all the ups and downs. Her patience and understanding during all the late nights, long weekends and missed events, has allowed me to achieve my goal. I can't express enough my appreciation, respect and admiration for her during this time. 


\section{Table of Contents}

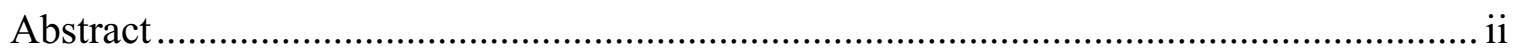

Acknowledgements ....................................................................................................

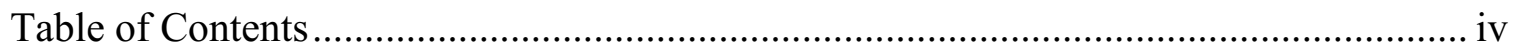

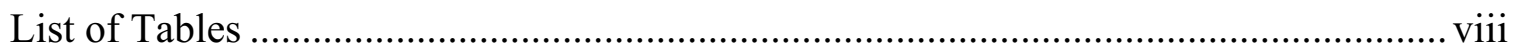

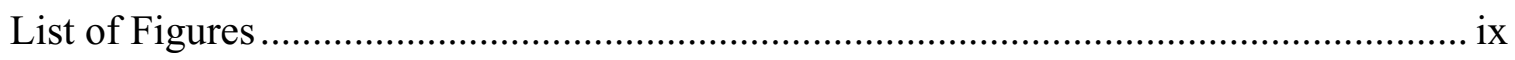

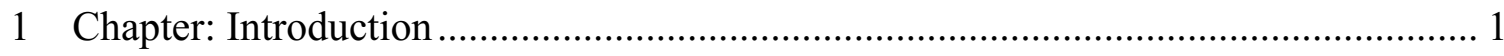

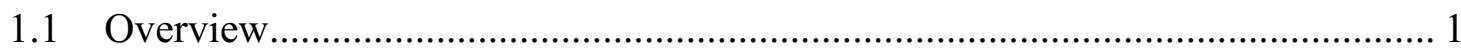

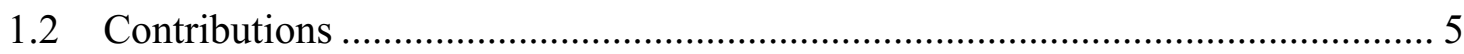

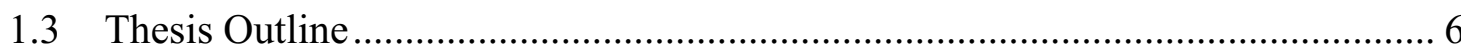

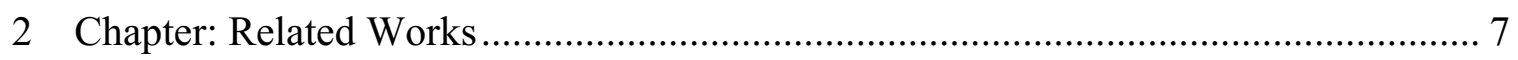

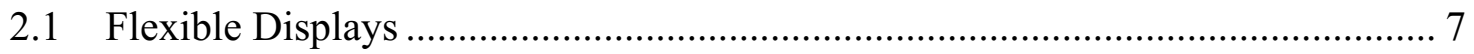

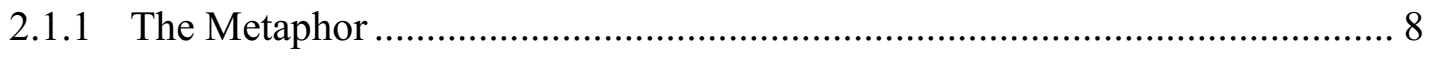

2.1.2 Prototypes and paper based inspiration ................................................... 9

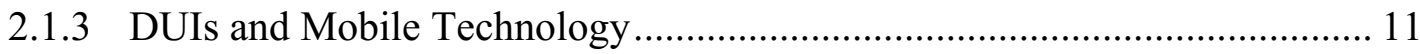

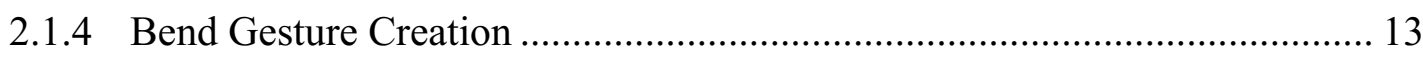

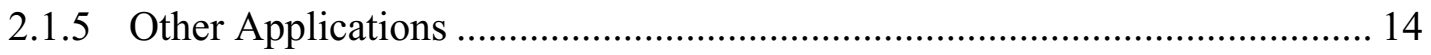

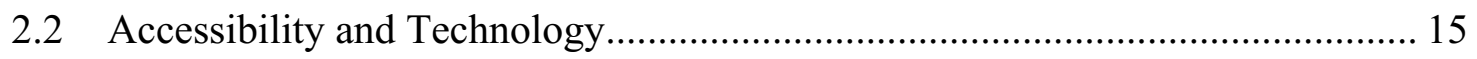

2.2.1 Technology and the Visually Impaired .................................................. 16

2.2.2 Non Visual Input and Interactions ......................................................... 16

2.2.3 Touch Gestures, Screen Readers and Apple VoiceOver............................... 17

2.2.4 Designing Gestures for the Visually Impaired ............................................. 18 
2.2.5 Feedback and System Response.............................................................. 19

2.3 Haptic Feedback and Stimulus ................................................................... 21

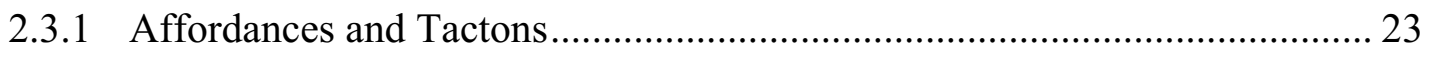

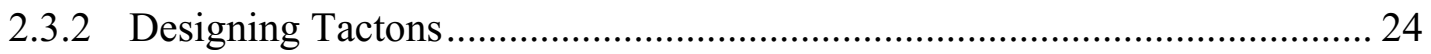

2.3.3 Designing with Tactons and Tactile feedback ............................................ 25

3 Chapter: Study 1: Tactons and Bend Gesture Learnability ……………………...... 28

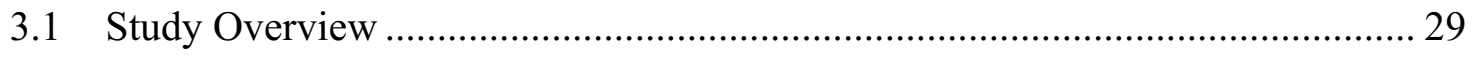

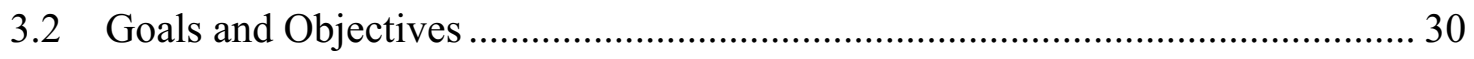

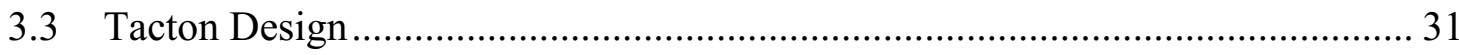

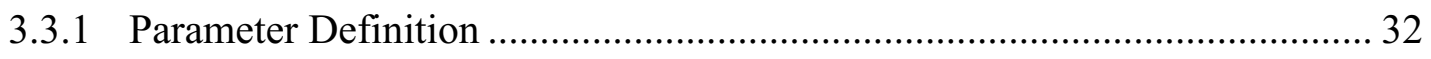

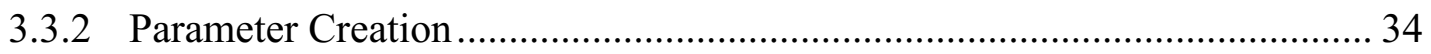

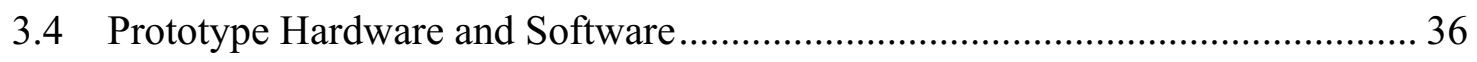

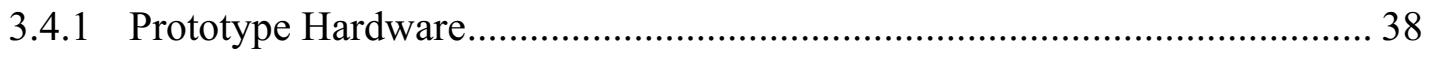

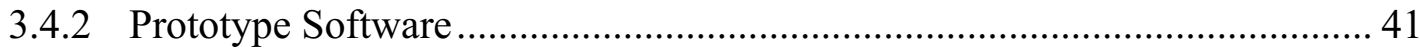

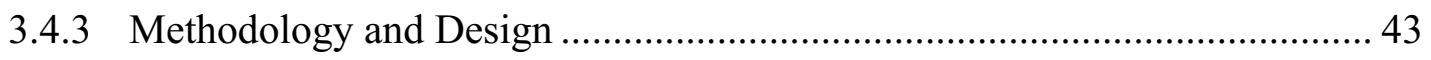

3.4.4 Study Breakdown .................................................................................. 44

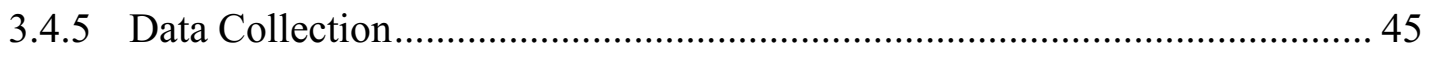

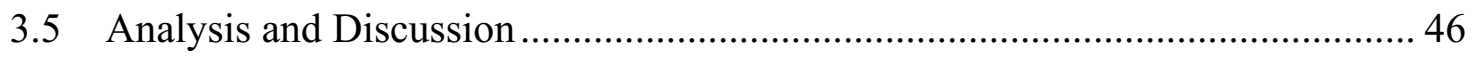

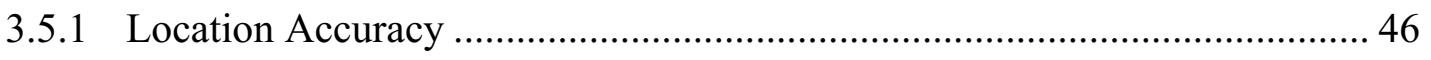

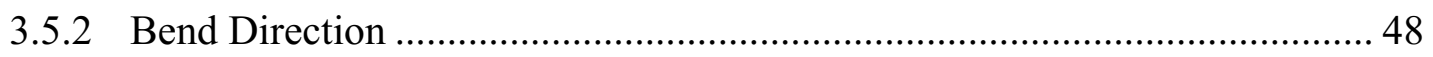

3.5.2.1 Bend Direction Association................................................................ 49

3.5.3 Mapping Confusion............................................................................... 50

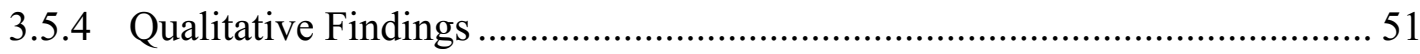




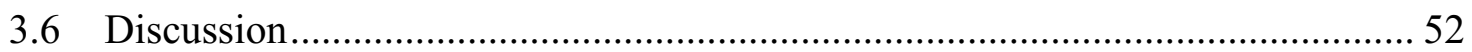

4 Chapter: Study 2: Bend Vs. Touch Gestures ........................................................... 55

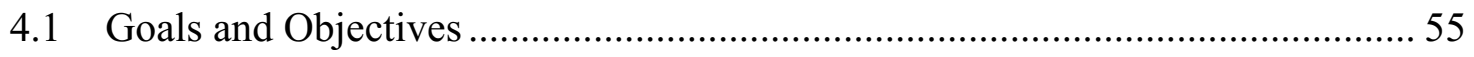

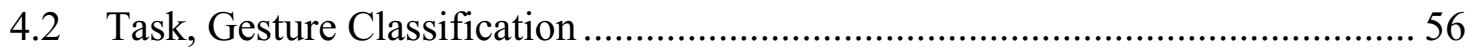

4.2.1 Swipe Gesture Classification ................................................................ 58

4.2.2 Bend Gesture Classification .................................................................. 59

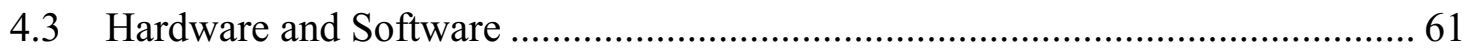

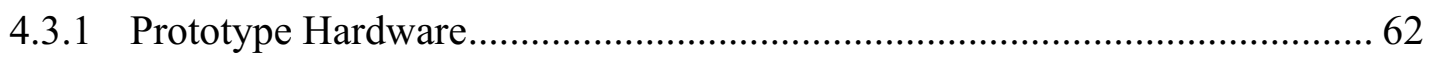

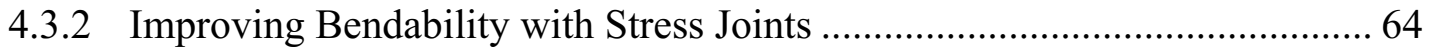

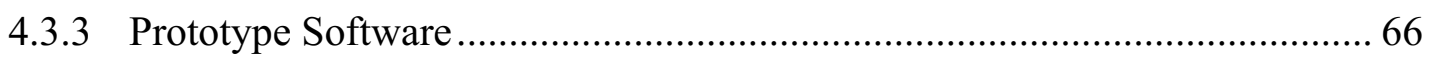

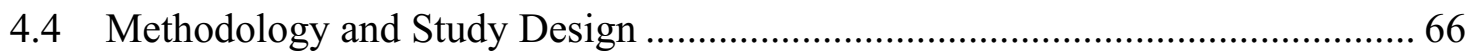

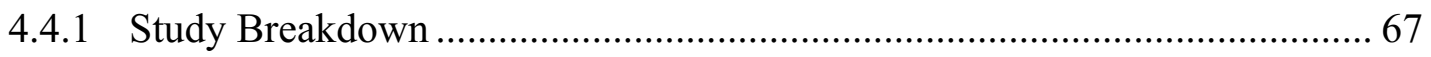

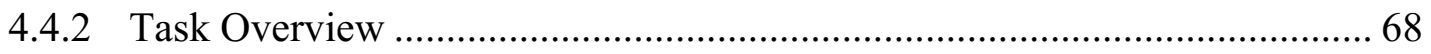

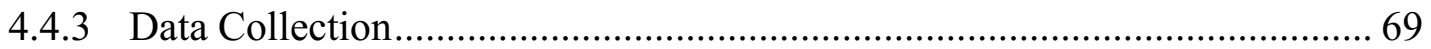

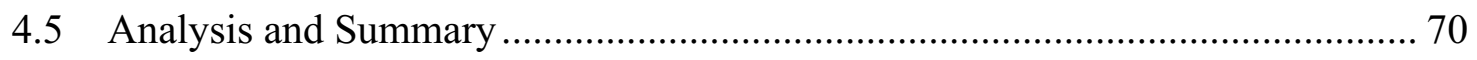

4.5.1 Task Completion Time......................................................................... 70

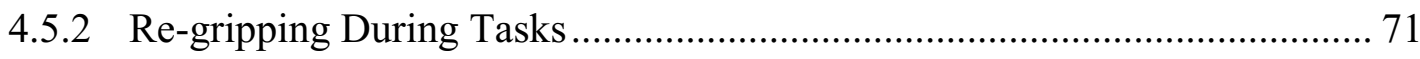

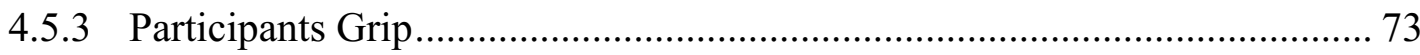

4.5.4 Bend and Touch Preference .................................................................... 74

4.5.5 Learnability of Bend and Touch Gesture Mapping...................................... 75

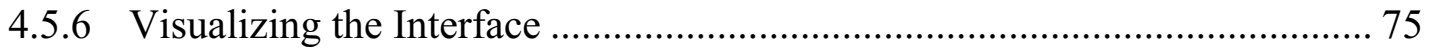

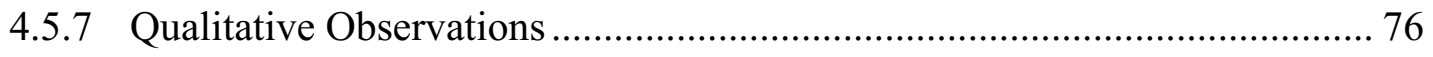

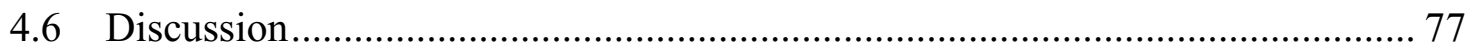


Discussion and Contributions

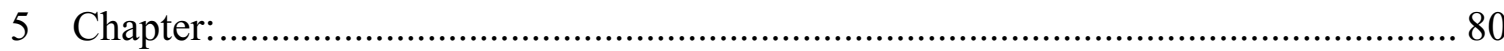

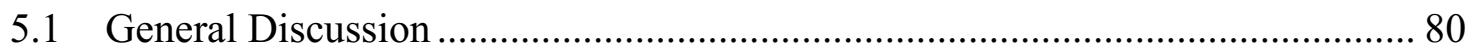

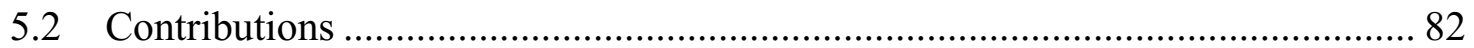

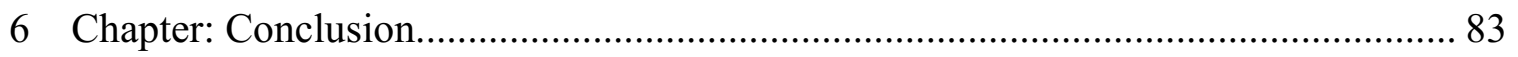

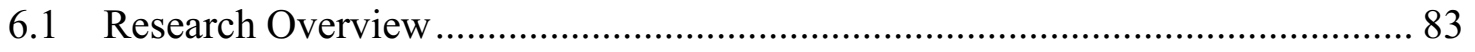

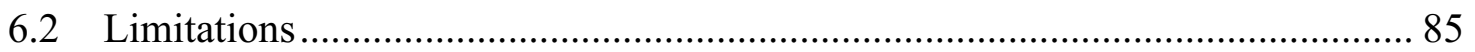

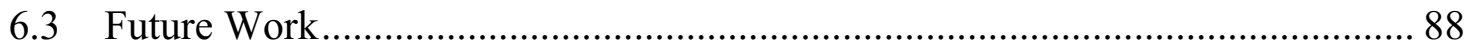

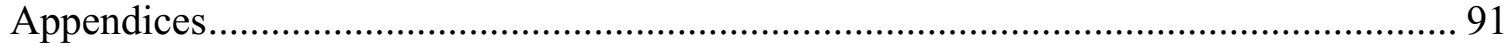

Appendix A Full list of Tacton designs ........................................................... 91

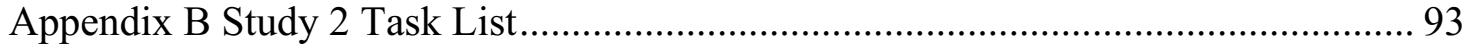

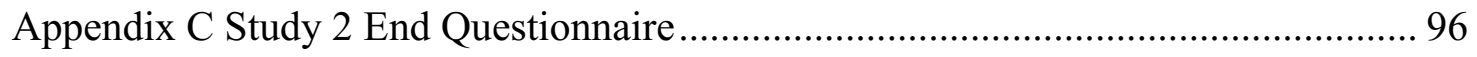

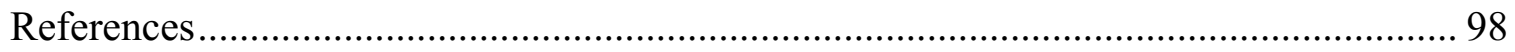




\section{List of Tables}

Table 1 Breakdown of each parameter variables including: motor combination, duration, waveform, intensity and location. (g) is the amplitude measurement controlled by the

voltage passed through the motor

Table 2 The confusion matrix for the statistically significant parameter: motor number for each of the location prompts. The bold numbers represent the correct location choices made by the user.

Table 3 The confusion matrix for the statistically significant parameter combination: motor number and duration for each of the location prompts. The bold numbers represent the correct location choices made by the user. 48

Table 4 Complete breakdown of system behaviour and user gestures. 59

Table 5 Complete breakdown of system behaviour and user gestures. 60 Table 6 Outline of all possible Tactons presented to the user. Pulse number refers to the Tacton ID number referenced in the pre-packaged software [56]. 92 


\section{List of Figures}

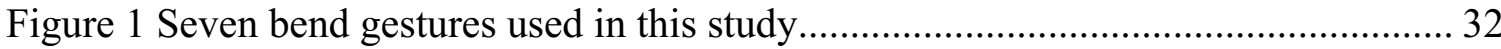

Figure 2 Illustration of the formula used to create each Tacton. Location + Duration +

Intensity Start to End + Waveform + Motor Combination............................................ 35

Figure 3 Simplified charts, visualizing a complete set of single Tactons for a single

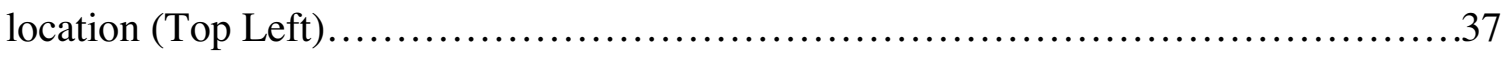

Figure 4 Study 1 prototype setup, including 1) Arduino Leonardo board, 2) 12 digit

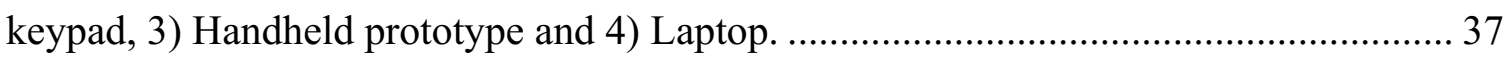

Figure 5 Capacitive Touch Keypad and Haptic Motor wiring. 1) cardboard prototype with 4 haptic motors, 2) Adafruit DRV2605L Haptic Motor Controllers, 3) Breadboard, 4) MPR121Touch Chip, 5) Keypad used to enter Tacton numbers, 6) Arduino Leonardo Board. Shaded area indicates the silicone prototype.

Figure 6 Illustrates the original flat prototype (a) that created space between the motors and palm. Further refinement of a concave shape $(b+c)$ maximized contact with the participant's hand and increased the identification of the vibrations................................ 39

Figure 7 Evolution of motor combinations, placement and orientation .......................... 40

Figure 8 Web App: "Location” interface..................................................................... 42

Figure 9 Web App: "Location/direction” interface ……….............................................. 42

Figure 10 Final prototype setup from two angles. .......................................................... 43

Figure 11 Illustrates participant's bend selection based on motor combination and

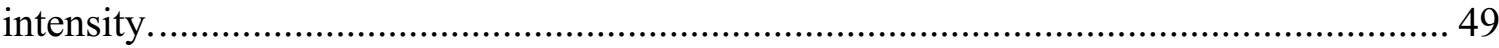

Figure 12 Mapping of participants Tacton feeling to bend direction agreement.............. 50

Figure 15 Six bend gestures performed by participants................................................... 60 
Figure 16 Overview of the 4 prototype setup components. 1) Arduino Leonardo board 2) Bend Prototype 3) Touch Prototype 4) System providing Audio Feedback.

Figure 17 Capacitive Touch Pad and Bend Prototype. 1) Bend prototype with 4 bend sensors, 2) Breadboard, 3) MPR121Touch Chip, 4) Touch Prototype with capacitive pad 5) Keypad used to enter Tacton numbers, 6) Arduino Leonardo Board. Shaded area

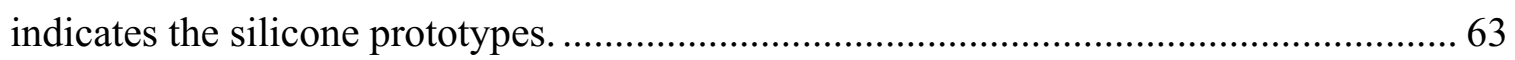
Figure 18 Images of strain relief designs incorporated into cables. 64 Figure 19 Construction of various prototypes testing groove location, depth and material density. 65

Figure 20 The screen and setup to create the visually impaired environment. 69 Figure 21 Average bend and touch completion times (milliseconds) for each of the 3 task sets. With Standard error bars. 71

Figure 22 Typical user flow for participants completing an action task for deleting an email. 72

Figure 23 Illustrates some of the common hand positions participants used during the study. The top row illustrates touch hand grip and the bottom bend.... 73 Figure 24 Distribution of Likert Scale responses for participants when asked to rate their comfort level when using bend and touch gestures. 74 Figure 25 Distribution of Likert Scale responses for participants when asked to rate their ease of learnability when using bend and touch gestures. 75 Figure 26 Distribution of Likert Scale responses for participants when asked to rate their understanding of focus placement when using bend and touch gestures. 76 


\section{Chapter: Introduction}

\subsection{Overview}

Living life in complete darkness or through a blurred filter is a challenge faced by millions every day. With one of the five senses severely impaired, these individuals are forced to depend on the remaining four to interact with and manipulate the world around them. No longer able to rely on visual cues or stimulus to understand when to cross the street or what object to pick up, they adapt and utilize touch, taste, smell and hearing to create an imaginative model of their current environment. These challenges traverse all aspects of life and require unique ways of viewing the world.

Although technology and specifically smart phones are a relatively new addition to our daily lives, they have changed the way we live, work and view the world around us. Now, an almost ubiquitous Internet connection enables constant communication with friends and family, and access to an unlimited amount of information and data. Unfortunately, this access often comes through a visual channel in the form of graphical user interfaces (GUIs) and touch screen displays or computer monitors. For users who cannot rely on this sense for consumption, access becomes much more difficult and exists through other outputs such as haptic or audio.

To improve accessibility to the Internet and information for the visually impaired, a range of technologies have been developed and studied. From experimental way finding methods using vibrations [1] to industry developed and implemented solutions such as Apple Inc's VoiceOver [51], they all strive to improve accessibility, user interaction and the overall experience for the visually impaired. 
Interacting with today's technology for the visually impaired comes with a unique set of challenges. The design of devices to deliver rich GUIs through retina displays encased in a sleek modern industrial design with the latest materials creates visually stunning experiences that appeal to billions around the world. Unfortunately taking away this visual component and asking some users to interact and perform touch gestures on a smooth cold, flat surface provides little context of the GUI that sits below the screen. With no tactile reference to anything other than edges or corners, they must rely on audio cues from a screen reader to understand the current page and perform generalized swipe and tap gestures to navigate lists and make selections.

Exploration into other forms of non visual input such as braille keyboards [3, 4] and speech [2] have seen positive results, but we explore a more tactile way of interacting with technology, one that would allow a user to physically manipulate the device in different ways to interact with an application. Bending and squeezing to navigate and make selections through easily locatable parts of the device, such as edges and corners, could better suit these visually impaired users.

This deformable method of interaction has yet to be researched within the area of accessibility, though we believe it has potential application for visually impaired users. Bend gestures and deformable user interfaces (DUIs) have been widely explored $[17,24,27,29,41,43,47,48]$ and require the user to manipulate and bend certain parts of a computing device, such as a Smart Phone, to interact with an interface, for example: bending a corner up or down to navigate up or down a list of emails.

This direct manipulation of physical objects is a basic tactile interaction we take for granted every day. The ability to deform, bend and shape items to suit our ever 
changing needs, allow us to customize objects through physical manipulation and connect with inanimate objects on a much more personal level. Paper is a classic example, ever since its invention around $100 \mathrm{BC}$ in China with the sole purpose of padding and protecting valuable objects during shipping [19], its usage has quickly progressed to a tool for documentation and communication, revolutionizing the world. As the function of paper has changed, humans have continually customized and physically manipulated it to create a variety of different uses beyond its initial intent. Bending in half to create cards, binding together to make books, dog earring the corners to identify reference points or folding into airplanes as toys, this physical manipulation by users allows for a more customized experience and creates a closer connection to the object [42].

This type of enhanced tactile interaction could be utilized within today's technology and specifically with visually impaired users as a novel way to interact with a screen reader or other audio based feedback tool. We further explored this unique concept to better understand if this tactile experience enhanced the usability and accessibility of technology for visually impaired users through tactile feedback and a novel deformable interaction model. First, we studied if participants could be introduced to bend gestures through tactile vibrations during the learnability stage, and second, if bend gestures could improve the general usability of mobile technology such as smartphones and VoiceOver.

Both studies used sighted participants with simulated vision loss, by hiding the device behind a screen. We understand the unique challenges faced with visually impaired users cannot be fully replicated this way. They experience the world differently and through heightened senses and understand, experience and view things very differently. However, we selected to use sighted users with no prior experience in either 
VoiceOver or pre-defined mental models and experiences with non-visual based interactions to ensure the most un-biased results.

Our first research goal in this thesis is to explore the learnability of bend gestures through tactile means and identify if certain types of haptic stimulus intuitively map to bend gestures. Haptics are used in most smartphones today but limited to basic alerts or notifications and generalized across the whole device. Past work includes exploration into haptics as a method of communication $[15,16]$ and the creation of what are called Tactons (tactile icons) $[4,7,16,20,33,38,49]$. No research that we are aware of has explored the mapping of Tactons to bend gestures, which thus became the focus of the first study. We explore this learnability concern for the visually impaired, but in the context of bend gestures and DUIs.

We conduct an experiment where participants are presented with unique vibration patterns and asked to pick a bend gesture they felt it prompted them to perform; comprising of a gesture location (top left, top, top right) and direction (up or down). A prototype generating 48 unique Tactons based on 5 parameters: waveform, intensity, length, number of motors and location allow users to pick one of the three sets of bend gestures through an on screen interface. We analyze the results to determine the effects of each parameter on accuracy of location choice and agreement of bend direction.

Our second research goal is to compare the effectiveness of bend gestures to touch as a primary form of input, while receiving audio feedback in a device occluded environment. 
Two iPhone size prototypes allow participants to blindly perform bend and touch gestures, navigating a series of interfaces to perform basic tasks. An HTML app provides audio feedback to the participant as they navigate this interface.

We analyze the results to determine speed of task completion for each of the input methods across task groups as well as participant's preference for the overall experience. We conclude by discussing findings from each of the two studies, including positive user feedback from the use of bend gestures within a visually impaired environment. This second study explores user interaction and overall experience for the visually impaired when interacting with screen reader software such as VoiceOver.

Although we conducted the studies in reverse order, the comparative study first then the haptic study, we present them in this order to create a narrative that begins with the perspective of learnability, followed by the usability of bend gestures.

\subsection{Contributions}

We contribute to bend gesture research by exploring the validity of applying bend gestures as a primary method of interaction for the visually impaired and the application of haptic stimulus in the learnability of these gestures. The first study identified a significant consensus among users for specific types of Tactons as non-visual prompts for bend gestures. This contributes parameter guidelines that can be used to design Tactons for future study in the learnability of bend gestures.

Although, findings from the second study identify no significant difference between bend and touch for task completion time, we identify significant preference among users when performing bend gestures as a form of navigation input in a non-visual setting. We also present improvements in the industrial design of potential bendable 
phones that include recessed areas along the back of the device. These grooves create weaker joints, producing more defined bends when performed by users.

\subsection{Thesis Outline}

This thesis is divided into 5 chapters. The first, introduces the overarching area of study and objectives for this research. The second chapter provides an overview of related works in deformable interfaces, accessibility and haptics as it relates to this thesis. The third chapter provides an overview of the first study and the learnability of bend gestures, introducing goals, objectives, Tacton creation, design and development of the prototype hardware and software, methodology and finally analysis and discussion. The fourth chapter provides a similar overview of the second comparative study between bend and touch. This includes, introducing the goals, objectives, task gesture classification, prototype hardware and software design and development, methodology and finally analysis and discussion. The final two chapters presents the findings, limitations faced during the research, ideas for future work and an overall discussion. 


\section{Chapter: Related Works}

With the main research goal of exploring bend gestures and their application in technology with visually impaired users, we first review the previous work completed in the research areas of deformable interactions, accessibility and haptic stimulus.

Bend gestures have been widely researched with findings recommending gesture location, direction, classification and device size [27,29,41,47]. Our goal within this literature review is to understand the parameters best suited for creating a set of basic bend gestures that could be peformed on an iPhone sized device for basic navigation and selection.

In addition to the bend gesture review, related works pertaining to accessibility and the visually impaired helped provide an overview of the usability struggles faced when using existing software and hardware as well as other explorations in alternative forms of input. This section also identifies usability flaws in existing approaches that could be solved using bend gestures.

Finally, a review of literature in both the application of haptic stimulus and the design of Tactons provides insight into the conceptualisation and execution of the second study. From the literature, the first study's methodology is defined along with the parameters used when designing the haptic prompts.

\subsection{Flexible Displays}

Allowing the user to bend, stretch and shape a deformable object to interact with a computer and provide input into a system, is the overarching tenet that defines this area of research. 


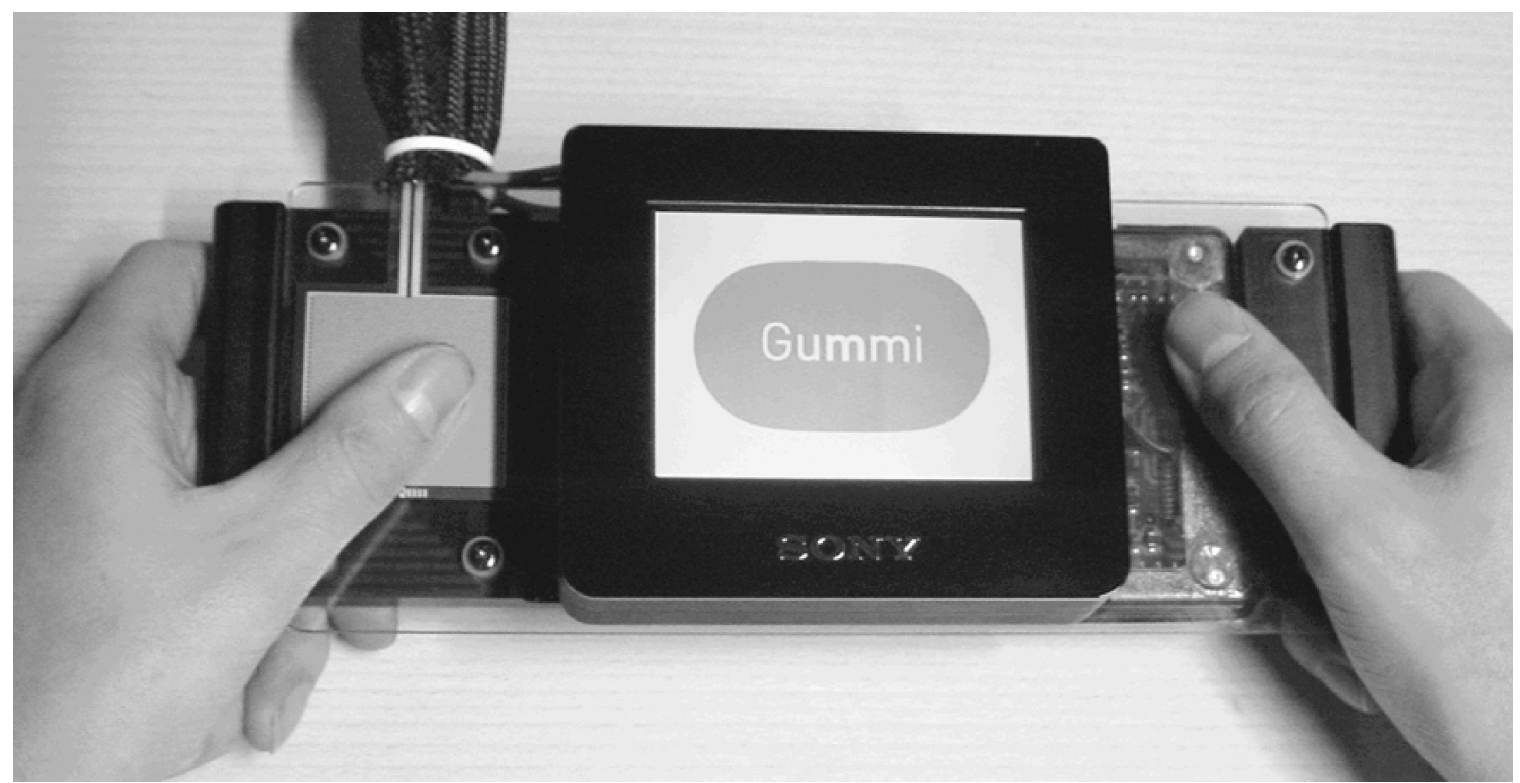

Figure 1 Gummi bendable prototype developed by Schwesig et al.

Schwesig et al. were one of the first to explore the concept of bendable computers with Gummi in 2004 [41]. The Gummi prototype (Figure 1) accepted physical manipulations by the user as the primary source of interaction and input into a computer. Originally conceived as a credit card sized bendable device, they produced a simple prototype with a flat rigid screen augmented by a bendable component that allowed users to perform two bend interactions: up and down. Along with the prototype, they introduced a set of interface paradigms such as scrolling, selecting and text input. This work and their findings set the stage for future research in bendable displays and proved that bend gesture interaction is a viable form of input when compared to other sources.

\subsubsection{The Metaphor}

Like most new technology introduced to mainstream users, deformable technology and bend gestures requires the user to learn and conceptualize new patterns and mental models. Unlike Graphical User Interfaces (GUIs) where the user is presented 
with on screen buttons and interactive elements, Natural User Interfaces (NUIs) such as gesture, tilt, voice, or bend, require the user to memorize certain gesture sets to effectively interact with the device. As with most user interfaces, these learnability issues can be eased with the use of metaphors when building mental relationships between preexisting patterns and new concepts [52].

This use of metaphors within interaction design has existed since the birth of the first graphical user interface and the Xerox Star [18]. As computers were being introduced into the workplace environment for the first time in the early 80's, Xerox introduced a new interface concept of iconography and digital pages or windows to represent a digital office. This approach allowed users to relate and better understand new concepts and interaction techniques based on existing knowledge of the office environment instead of learning a completely unfamiliar and complex computer language in the form of command line.

This metaphorical approach and relationship to past experiences can also be seen throughout the literature $[11,17,28,43,48]$ and work exploring bendable computers. Because of the innate physical relationship to paper in size and manipulation, a substantial amount of work in the form of e-readers and paper-sized prototypes has been completed $[11,17,48]$.

\subsubsection{Prototypes and Paper Based Inspiration}

Lee et al. [28] allowed users to define bend interactions while interacting with different materials and found that users defined these gestures based on common daily paper actions. Holman et al. [17] developed a prototype that projected an interface onto physical paper and traced the shape and position of each page to translate into 
interactions. Users were able to interact with multiple physical pages in a tracked environment using touch, bend or sorting to interact with the digital projection. Their concept pushed the boundaries of the digital office and incorporate the tangible nature of physical paper into a digital environment.

Watanabe et al. [48] created BookiSheet, another direct application of the physical paper experience to a digital environment. They focused on a small set of bendable gestures that allowed users to interact with a digital book. Constructing a prototype out of thin plastic sheets and bend sensors they compared their BookiSheet with a physical book, touchpad, mouse wheel and arrow keys with the aim of evaluating page scrolling. Results indicated that the bend interactions performed equally well to other interaction techniques.

Gallant et al. [11] also explored page navigation and bend gestures within the context of a 3D prototyping tool. Allowing users to manipulate a 3D digital object using a foldable piece of cardboard, they tracked deformations with an infrared camera and reflectors. They also explored bend gestures performed on a single page such as folding, squeezing and bending the top corners as well as multi page interaction. As seen in most prototypes used in deformable experiments [17], a projector is used to create a visual representation of a screen directly on a flexible surface.

One of the more common problems experienced with this approach is the user's hands obstructing the projection of the display. Steimle et al. [43] introduced a refined projection method that not only captured smaller deformations of the page but reduced this hand obstruction. With this new implementation, they also explored highly deformable materials allowing the user to perform precision bends while interacting with 
the interface. Results showed that participants could easily use these highly flexible displays. Lee et al. [28] also explored how users manipulate and interact with different flexible materials through bending, folding or stretching. They provided users with 11 commands and 3 different materials, concluding that users preferred a more flexible material to interact with. Kildal et al. [26] also identified that not only did users prefer more flexible materials, but, performance significantly improved over stiffer materials.

\subsubsection{DUIs and Mobile Technology}

With the mobile revolution in the late 2000's and the release of a range of new smartphones, research investigating the application of deformable interactions within the mobile context became widespread.

Herkenrath et al. [15] were among the first to explore the concept of mobile devices and possible bend gesture interactions as a source of input.

They developed a prototype that allowed a user to deform corner edges and parts of the phone to perform interactions, and proposed a study to compare bend gestures to other common interaction techniques such as touch. 


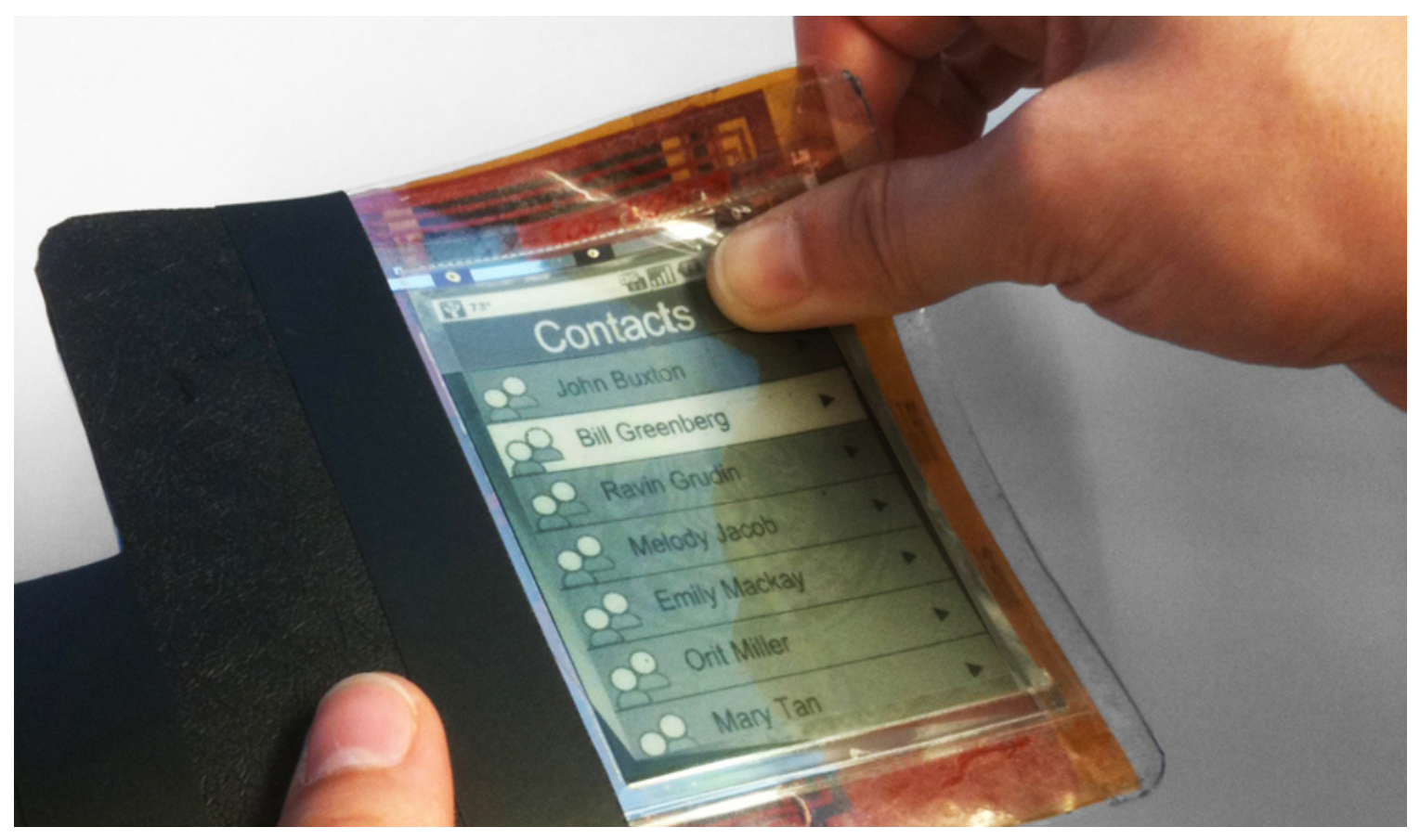

Figure 2 PaperPhone prototype developed by Lahey et al.

PaperPhone (Figure 2) also explored bend gestures within a mobile device context. Lahey et al. [27] conducted a study where users created their own bend gestures to perform basic interactions on a flexible screen. Their prototype was the first to utilize a flexible e-ink display in combination with bend sensors. This created a more realistic environment in which to conduct experiments instead of past prototypes that used projection or simulated paper devices. Results showed that although participants consistently conceptualized similar bend gestures such as corners or sides, there were inconsistent mappings to interface actions. Kildal et al. [25] also developed a prototype with a flexible display to explore mobile applications for bend gestures. This tablet sized prototype required holding with two hands in a landscape position to effectively perform interactions such as twisting and folding. 
Past work utilize all sizes of prototypes, from large paper dimensions to smaller hand held sizes. Lee et al. [29] explored the effects of these different sizes on the classification of bend gestures. Their findings identified smaller devices are preferred by participants, requiring less effort to manipulate and could be performed using one hand. They also noted that $28 \%$ of users expressed their preference for a touch interface over bend when using the larger screen but no preference for the smaller device.

\subsubsection{Bend Gesture Creation}

Other researchers have attempted to classify and propose guidelines for designing bend gestures. Warren et al. [47] were the first to propose a set of comprehensive parameters such as utilizing the top corner for frequent tasks, restricting the level of magnitude to two when creating bend gestures and limiting factors such as size or angle to reduce confusion among novice users. This classification is used to define our bend interactions with the parameters of location and direction.

Girouard et al. [13] were the first to explore the specific area of one handed bend interactions on a smaller form factor. They proposed a series of bend gestures and tested performance between hands. Their findings identified up gestures being preferred to down, the top two corners and center squeeze being the fastest to execute and the primary corner as the one closest to thumb. For example, if the user is right handed the top right corner would be the fastest over the top left where as a left handed user would prefer the top left. 


\subsubsection{Other Applications}

In addition to research focused primarily on bend gestures and DUIs, Kildal et al. [24] explored the combination of bend gesture and touch interactions. Using a prototype that allowed users to perform both bend and touch interactions, users performed a series of tasks that required them to interact with an on screen photo manipulation application. Although no significant difference was found comparing task completion times for multi touch or a combined approach of bend and touch, participant's preferred the combined approach and noted a better sense of control than touch alone.

Other work by Tarun et al. [44] explored the concept of a deformable device that, based on its shape, would change function.

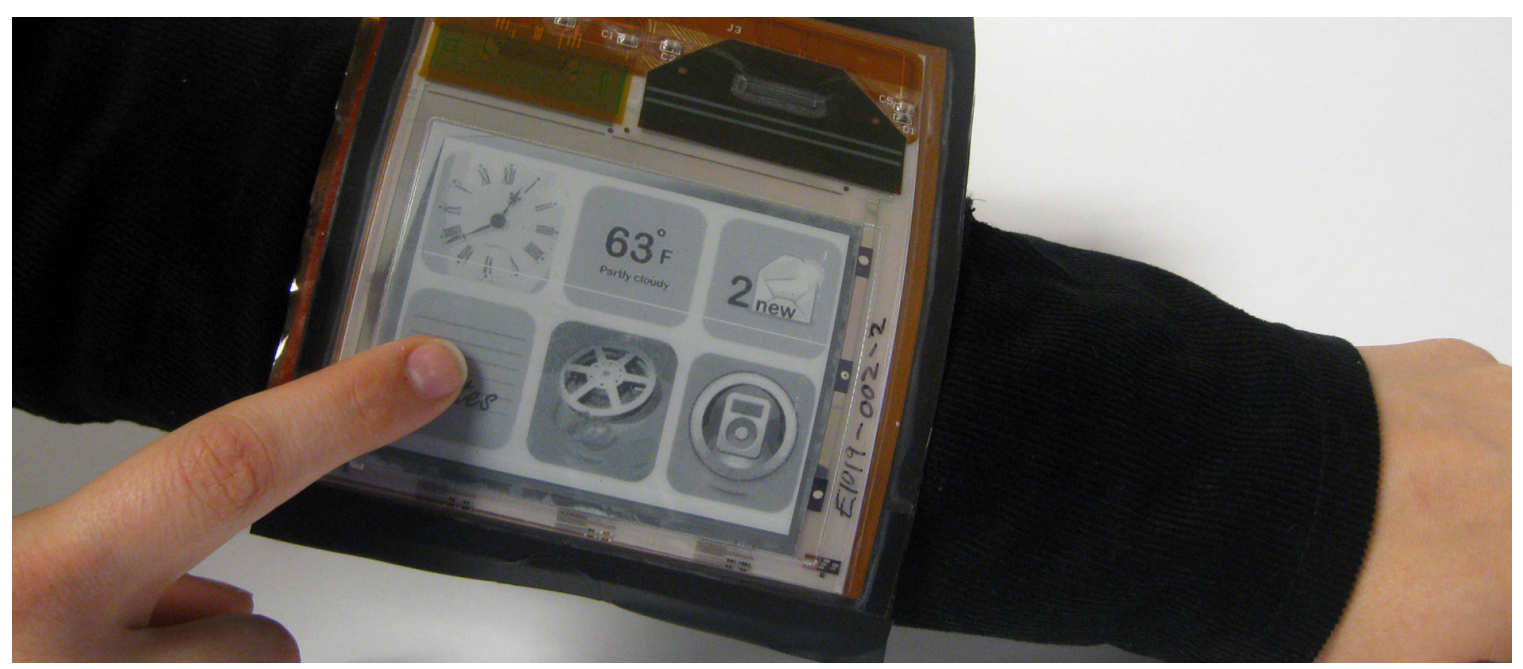

Figure 3 Snaplet prototype developed by Tarun et al.

They developed Snaplet (Figure 3), a wearable prototype developed using a flexible e-ink screen and sensors to detect the shape of the device. When in a convex shape and worn on the forearm it would transition into a watch or music player. When flattened it could be used as a traditional PDA with a stylus and when bent to a concave shape it became a phone. They further outline the benefits of this concept and state that a 
changing shape offers users a direct non-visual affordance to the current state of the device. This has interesting applications for the visually impaired that are unable to receive visual cues from a screen.

This overview introduces the work completed in the domain of DUIs. We use it to define parameters best suited for creating bend gestures for visually impaired participants. Within the literature common tenets emerged that define gesture creation, such as the importance of the top corners of the device. Best used for frequent tasks [47], and the fastest to execute [13], the top two corners of the device become the ideal location to perform gestures for common tasks such as navigation and actions. Further defining these corner interactions, the primary corner can be identified as the one closest to thumb and best suited for most frequent tasks [13]. These guidelines, best practices and key findings define the set of 7 gestures used as the foundation of both of our studies. Other research $[24,44]$ shape future work and conceptual adaptation of smartphones and deformable technology with visually impaired users.

\subsection{Accessibility and Technology}

Over 285 million people are estimated to be visually impaired worldwide [46], and with technology designed primarily for the sighted population, it often proves more challenging for these individuals to effectively interact with technology that could drastically improve day to day living. Over the past several years, the mobile revolution of touch screen devices such as the iPhone and iPad, has opened the door to a world of information, applications and content that can be easily accessible from any location. 


\subsubsection{Technology and the Visually Impaired}

This widespread adoption of mobile technology exists for all types of users. Kane et al. [21] conducted a qualitative study with 8 blind mobile device users and found that most respondents carried on average 3.6 devices such as mobile phones, laptops, Braille PDAs, and audiobook players to perform specific tasks. Every respondent encountered usability issues with touch screen devices with key problems being "learning where objects were located on the screen" and "accidentally activating certain features on the touch screen."

Unfortunately users with various degrees of visual impairments struggle to interact with these touch interfaces due to the lack of non-visual feedback through audio, haptic or physical buttons $[21,30]$. The many benefits of a touch screen often become a barrier to the visually impaired without assistance of a sighted person, hardware attachments or additional software, such as Apple's VoiceOver [51], Talk Back [53] and Blind Square [54].

\subsubsection{Non Visual Input and Interactions}

Interacting with technology for any user can be reduced to two basic functions; a user performs an action that provides input to the system, while the system provides feedback to the user. For visual users, this input comes in many forms such as GUI buttons or sliders, with feedback presented in the form of animations and new information appearing on screen. The majority of mobile technology is designed with this in mind with touch interfaces providing rich graphical representations of the system. 
For blind users who are unable to interact easily with GUIs, there are a variety of alternate input techniques available, such as Braille keyboards, voice and touch screen augmentations.

Rantala et al. [39] explored three unique interaction methods for reading Six Dot Braille characters on a smartphone using piezoelectric actuators under a touch screen. They found that participants could accurately distinguish single characters by $91-97$ percent. Six Dot Braille is an almost universal representation of language in tactile form with each character in an alphabet represented by a series of raised dots. This allows a visually impaired user to quickly scan these raised dot sequences to read.

Input is a difficult challenge for visually impaired users $[21,30]$ as traditional keyboards are small and rely heavily on visual layout and cues. Frey et al. [10] explored a novel way for users to enter characters using the braille language on a touch screen smart phone. Their concept contained 6 quadrants on the screen and when faced away from the user, they could use three fingers on each hand to enter the 6 dot formations of each character. Although they conducted a more informal evaluation with industry experts, their concept showed promise as a cheaper alternative to existing Braille keyboards.

\subsubsection{Touch Gestures, Screen Readers and Apple VoiceOver}

Although these explorative concepts looked to replicate the existing braille language and input techniques with current technology, other approaches such as Slide Rule developed by Kane et al. [21], and VoiceOver by Apple adopt a gesture based interaction approach. The basic concept for these non visual based interaction techniques allow users to perform a series of gestures to navigate lists within an application and receive audio feedback based on the position within the application. 
It is interesting to note that Kane et al. published their findings in 2008, a year before Apple released VoiceOver for the iPhone3GS. During their comparative study, their findings also concluded that their "exploratory" method of navigating a touch screen resulted in more errors made by users when compared to a Pocket PC that forced users to navigate a list systematically.

The release of VoiceOver by Apple, provided a single piece of mobile technology that could grant access to a range of applications, content sources and functionality. VoiceOver opened the technology door for the visually impaired and allowed access to the newly emerging smartphone market. Soon after its release, multiple UI evaluations $[8,30]$ concluded that although VoiceOver makes devices easier to interact with for the visually impaired, there are still usability concerns that can be addressed. Lack of logical navigation order, inconsistent focus, lack of orientation and conflicting app and system controls can lead to an overall poor experience. Another issue identified in these evaluations that still exist today is text editing. From a usability perspective visually impaired users find the keyboard extremely laborious to use and entering text is prone to error $[8,30]$.

\subsubsection{Designing Gestures for the Visually Impaired}

Although touch screen interfaces offer a range of benefits and almost infinite graphical and gesture possibilities, it is important to consider how the visually impaired use touch gestures as a form of input. Kane and Ladner [22] compared how blind and sighted users interact with touch interfaces through a user-defined study where participants were asked to create gestures that mapped to common tasks. Their results indicate that blind participant gesture properties included more strokes, preference for the 
edge or corner of the screen, use of more abstract shapes and the invention of more multi touch gestures. Blind participants used multi touch in an interesting way in which they used one finger placed at a certain location while the other finger performed a gesture. This helped to reduce any conflict between similar gestures.

Their findings lead to recommendations for designing touch interactions, which include:

- Avoiding the use of symbols found in print.

- Favor edges and corners.

- Reduce need for specific location accuracy.

- Limit time restrictions for gestures.

- Use familiar patterns and layouts from other applications.

Although various solutions continue to be designed to aid the visually impaired in interacting with technology, receiving feedback in any form can be equally challenging and present concerns around accessibility and usability.

\subsubsection{Feedback and System Response}

Feedback can exist in many forms, with the most common in technology being visual through a screen or projection. Unfortunately visually impaired users cannot utilize this feedback loop and must rely on their other senses to understand changes in the interface or receive updates from the system. This can come in the form of audio, haptic or other more experimental forms such as shape-changing [9]. 
Li et al. [31] conducted early work on the use of audio feedback to interact with a device. They developed BlindSight, an application that allowed users to interact with a phone keypad at any point during a conversation to perform additional actions such as creating a calendar event or adding contact info. Participants would touch certain keys to scroll a menu or input data into certain applications. Their findings identified that not only could users interact with the device without looking but provided several design solutions for better interaction such as raised dots on key number pads and increased spacing between keys. They also identified that participants preferred BlindSight over a visual based system while talking on the phone as it provided less of a distraction allowing focus to remain on the conversation.

Zhao et al. [50] designed a music player that used touch input and audio feedback and evaluated its effectiveness when compared to a visual alternative. An interactive wheel allowed users to navigate through a list while the focused item were converted to audio output. Users were asked to find a corresponding word when displayed, either visually or through an audio recording. They found no statistical significant difference between the audio and visual approaches which means that users can perform tasks equally well with both forms of output.

This substantial body of work conducted in all areas of accessibility utilizes different interaction techniques, and explores all types of feedback. Touch is the predominant method of input in today's technology and often the focus of usability studies and explorations due to the predominant market saturation. Other input alternatives do exist [40, 41], and we look to add to this work by exploring the use of bend gestures and their validity in this field. 
Since visually impaired individuals rely heavily on tactile feedback to understand the world around them, we wanted to explore a more tactile way of interacting with technology, one that would allow a user to physically manipulate the device in different ways to interact with an application. This exploration of DUIs and potential applications in the area of accessibility could create improved interaction models for applications such as VoiceOver, talkBack or Siri and allow these users to text, email or access a wealth of information on the internet with improved usability.

\subsection{Haptic Feedback and Stimulus}

Touch is one of the human bodies 5 senses that often goes unnoticed as we interact with the world around us. We rely so heavily on our hearing and vision to live, work and communicate with others that the feel of textures and objects often becomes overpowered and ignored [34]. One of the earliest senses to develop, it allows the body to perform intricate movements and interact with physical objects during our daily life. This haptic sense is comprised of two sub modules: tactile and proprioceptive. Tactile refers to direct stimulus to the skin through heat, pressure, vibration or roughness. Proprioceptive refers to the use of muscles, bones and the body to estimate physical object body positioning. For example, if you are holding a cell phone in your hand, the plastic and glass textures would be an example of tactile feedback, where the perceived position of cell phone in relation to your other hand and body would be an example of proprioceptive feedback [34].

Although these two types of feedback are important for any person, they are especially pivotal for the visually impaired. With limited or no vision, these individuals utilize both forms of tactile feedback to understand the world around them. Their reliance 
on touch to explore textures and forms within their surrounding environment helps build a mental picture to interact with. This reliance does not change with their use of technology and becomes even more critical to enable them to complete tasks and successfully interact with any smartphone or computer. Because technology relies heavily on visual feedback to inform the user of a change in function, they are rendered inaccessible and require an additional layer of feedback to inform visually impaired users. In current applications, this primarily comes in the form of audio output and screen reader technology.

Un-utilized within modern technology [34], haptic stimulus in the form of basic alert and notification vibrations can play a pivotal role in providing additional feedback to the user. Through the use of actuators and vibrating motors, devices can apply heat or vibration forces to certain parts of the user's body to relay rich forms of communication. These "Active haptic devices" [34] have been used in technology since the beginning of the 1990's but are still rudimentary in application. Minsky's textural explorations [35], completed work on haptic feedback with a prototype called "Sandpaper". Simulating different grades of sandpaper using haptic stimulus they evaluated participants feedback on the experience. Brooks at al. [5] also explored the augmentation of visual interfaces with haptic stimulus. This work by Minsky and Brookes is considered to be one of the first applied uses of haptic feedback.

These tactile vibrations often augment visual or audio experiences and invisible interactions to aid the user in the understanding the UI. This form of education is critical for users to understand how to effectively interact and communicate to the system. For 
GUIs this comes in the form of visual affordances and icons but for non-visual interfaces it becomes more challenging.

\subsubsection{Affordances and Tactons}

In the case of invisible or audio-based interfaces like the one studied in this thesis, they contain no visual prompts to guide the user through the interface. All interactions must be committed to memory and referenced for every interaction. This poses two significant problems to both the designer and user. First; how to teach a user what various speech or gesture based interactions are available and what they do, and second; how to ensure they remember to use them and in what context.

Norman [36] states that operating an interface at a basic level requires three dimensions, conceptual models, constraints and affordances. Affordances are indicators to the user that help identify its function or intended interaction. They can come in various forms including visual, auditory or tactile and apply to anything we interact with. Designed and incorporated into any fabricated artifact, their intent it to provide a prompt or understanding to the user on the intended functionality. Affordances play a critical role in interaction design to help users understand interaction patterns, remembering and performing hidden gestures such as touch, speech or bend. These affordances help in the way we process information, make decisions and interact with technology and influence the way the interface is designed.

Affordances play a critical role in teaching and prompting the user how to perform a hidden interaction or gesture, with limited mental effort. For visual based users this often comes in the form of icons or visual cues that help instruct the user, but for non-visual users the use of haptic icons or "Tactons" [4] can play a similar role. 
Tactons are haptic-based icons that like their visual counterpart help inform the user of the current state of the system. They are used in most technology today in the form of unique vibrations when you receive a text message or email, and like any component within an interface they must be designed to ensure the proper information is presented. There have been numerous papers $[4,6,7,19,35]$ on the topic of Tacton design, which can be used to shape the design and implementation of the Tactons used in the first study.

\subsubsection{Designing Tactons}

Brewster and Brown [4] were one of the first to coin the term "Tactons". Their paper outlines the foundation for designing Tactons and explains the different types of parameters used in their design. They identify these as amplitude, waveform, duration, rhythm and body location. Amplitude refers to the degree of change in intensity. Waveform refers to how the amplitude changes and comes in various types: wave, square or saw. Rhythm refers to the grouping together of tactile pulses and body location refers to the location of the motor when in contact with the body.

Brown et al. [6] also explored multiple parameters when designing Tactons including; rhythm, roughness, frequency, intensity and spatial location. Their study focused on three: rhythm, roughness and spatial location and presented variations of each to the user who in tern picked which notification they felt it best represented (Text message, email etc). They found that all all three parameters only produced a $47 \%$ success rate. But just combining location and rhythm resulted in a 95\% success rate. Overall, reducing the number of parameters is key to ensuring Tacton design is successful at communicating to the user. Kaaresoja and Linjama [20], explored vibration length and 
users perception of each Tacton. They identified that vibrations should be no less than 50 ms, but shorter than $200 \mathrm{~ms}$.

Brown et al. [7] also explored the application of musical techniques to Tacton design. Adjusting the amplitude through linear, exponential and logarithmic waveforms created a unique set of Tactons. Their objective tested which type is most effective and easy to recognize and found linear Tactons superior than the others. Hoggan et al. [16] also explored if meaning can be conveyed by haptic icons using different waveforms. After testing various Tactons with users blindly assigning meaning, they discovered that Tactons could have consistent meanings across users. For example, confirmation type notifications can often be presented by short rhythms, and central spatial location. Errors can be presented by very rough texture, and fast tempo. Progress Updates can be presented by circular motion, and long duration and warnings by very rough texture and fast tempo.

Qian et al. [38] explored the application of Tactons and their use as non-visual cues. Participants were presented with different Tactons, and asked to identify which pair was noticeably different. They found that Tactons should be consistent and static in rhythm with changes in pulse length were best noticed by participants.

\subsubsection{Designing with Tactons and Tactile feedback}

Poupyrev and Maruyama [37] were one of the first to explore tactile feedback to enhance a touch screen. They designed a PDA that, instead of displaying a visual response to the user, presented a Tacton produced by actuators in corners of the screen. Their results showed overwhelming positive feedback, and users commented this haptic feedback replicated the feeling of an actual switch. 
Sahami et al. [40] also explored if multi tactile feedback in different locations could benefit a touch experience. They embedded six motors along the sides of a device and tested how easy it is for users to identify different motors and patterns within these 6 unique locations. Their findings conclude that the corners of the device are most recognizable with top left at $73 \%$ and top right at $63 \%$. It will be interesting to compare these findings with our results to identify any similarities.

Buzzi et al. [8] proposed a similar concept of inserting vibration motors along certain parts of the device to identify certain on screen UI elements. Although not tested, they proposed a series of paradigms that would allow a visually impaired user to explore a UI and receive haptic feedback. Yatani et al. [49] also explored the use of Tactons but to prompt touch gestures instead of UI elements. They developed a curved sleeve that fit behind a phone to reduce the gap between the device and the user's palm, ensuring a more direct contact with the skin. After designing eleven different Tactons based on positional, linear and circular vibrations they determined that users could distinguish 10 different patterns at an $89.6 \%$ success rate on average except for counter-clockwise.

Azenkot et al. [1] also explored the use of Tactons on a mobile phone for turn-byturn way finding for visually impaired users. They conducted a comparative study between three different approaches: Wand, Screenedge and Pattern. The wand approach allowed users to point the phone in different directions and when the intended path is intersected the top of the phone will vibrate. Screenedge allowed user to touch parts of the screen and receive vibration feedback corresponding to the direction they need to walk. Finally the pattern concept generated different pulses depending on the direction the user needed to walk: one to walk forward, two to turn Right, three to walk back and 
four to turn left. They conducted the experiment with eight visually impaired users with error rates for each approach being; Wand: 9\%, ScreenEdge: 2\% and Pattern: 2\%. Four participants preferred pattern, three Screenedge, none for wand, and one preferred both pattern and screen edge.

This body of work demonstrates that haptic stimulus and Tactons can have a successful role and application in the augmentation of existing interaction patterns and interfaces. Past work shows the successful creation of Tactons that, when presented to participants, could prompt a swipe gesture in a certain direction [49]. We built on this concept, and explored if certain types of Tactons could prompt a bend gesture in a certain location and direction. Due to the tactile nature of bend interactions, the use of a tactile form of feedback could be applicable for visually impaired users in both the teaching of these hidden gestures and affordances during notifications and prompts. We utilize this past work $[4,6,7,16,20]$ to define parameters used in our Tacton creation, such as wave type, duration, intensity or location and set the stage for our first study and the design and implementation of Tactons within the context of bend gestures.

Our first study explores this concept and the design and implementation of Tactons within a visually impaired environment. Because no work has yet been completed to date the first step is to understand what types of Tactons users can identify, and what meaning they assign with respect to bend gestures. 


\section{Chapter: Study 1: Tactons and Bend Gesture Learnability}

NUIs such as tilt, gesture, speech and bend are invisible interactions to the user and require some level of training or education to instruct how to successfully interact with a given interface. Learnability often becomes challenging in these scenarios, as users are required to commit patterns and interactions to memory that will then be recalled during future cases. It is even more problematic for visually impaired users who are unable to watch instructional videos, see coach marks or onscreen visual affordances to help guide and learn new patterns and interaction techniques. Current solutions for these users to learn new interaction patterns often require dependence on a sighted user, or audio tutorials [55].

Although bend gestures have not been implemented into mainstream technology, it would be our assumption that a similar education process would exist. A user would still need to learn what parts of the phone they could bend and what functionality that maps to regardless of any visual impairment. They would also require non-visual prompting to complete certain interactions at specific times. Numerous alternative design solutions exist, including audio, physical demonstrations and haptic stimulus, and although all of these have potential, we see promise in the use of haptic stimulus to assist in the education of bend gestures when visual prompts are not accessible. While audio is a viable candidate as a tool for learnability, we wanted to explore a more tactile experience that would complement the physical nature of bend gestures themselves. Haptic stimulus is something you feel and experience through touch of your fingers or palm, and we wanted to explore this direct tactile relationship between vibrations felt by the user and their perceived understanding of what gesture it was prompting. Existing 
research demonstrates the effectiveness of haptic stimulus to prompt touch interactions [49], but we are unaware of any existing research that has explored their application with bend gestures.

\subsection{Study Overview}

Whether Tactons are used independently, or combined with audio feedback in training, coach marking or app notifications, they could both aid in the learnability of these interactions and help identify required gestures during regular use. A basic notification example could be as follows; the user is required to bend the top left corner of the device up to answer a phone call, instead of receiving an audio tone or a generic buzz the user could be prompted with a location based vibration that increases in intensity. The location of the vibration could remind the user which corner to bend and the intensity increase could prompt which direction to bend. Our objective with this study is to explore the use of haptic stimulus and how they are effective mapping to up and down bend gestures.

Behind the design of every Tacton, there is a stimulus and a context. As defined in previous work $[16,17,37,15]$, the stimulus component can incorporate a variety of different parameters including:

- Duration: Length of Tacton

- Amplitude: Intensity of vibration

- Rhythm: Grouping together pulse vibrations with gaps

- Melody/Wave Form: Different amplitude and pulse vibration patterns

- Roughness: Combining two variations in vibration amplitude 
- Spatial Location: Different locations of the vibration motor.

- Motor combination: The use of one or two motors.

Context, on the other hand, can be assigned by the designer or inferred by the user, with the objective that both the designer's vision and the user's perception align. Past work often assigns arbitrary meanings to parameters [6] such as types of notifications or urgency. Because these have no direct relationship to the type of vibration pattern, the user has no prior context or meaning and is left guessing or trying to remember the Tacton in question. Hoggan et al. state that "Ideally the mapping between meaning and icon should be as simple as possible and consistently interpreted in the same way among all users.” [16].

This study aims to explore Tacton designs that combine an appropriate stimulus with relevant meaning to a pre-defined set of bend gestures.

\subsection{Goals and Objectives}

Our goal during this study explored if any agreement existed among participants on bend location and direction when presented with a certain configuration of haptic parameters in the form of a Tacton. Using parameters to simulate up and down sensation explored if participants could map these to a bend direction. Intensity and motor combination directly explored this directional mapping. These up or down changes in intensity or the directional perception of up or down with the use of two motor combinations tried to create a tactile sensation of depth. Our intent explored what participants perceived as up or down and use any successful findings to further explore 
the tactile relationship between Tactons and bend gestures in the context of teaching or notification systems.

Designing Tactons for bend gestures have several possibilities to intuitively map certain parameters to the location and z-direction. Based on prior work, our hypothesis states that the location parameter of a Tacton can effectively tell the user which location to bend. The intensity, waveform, motor combination and duration can identify to the user which z-direction to bend. The objectives of this study are as follows:

A. Identify which parameters used in the creation of these Tactons can more effectively prompt the user to accurately select a bend gesture location (i.e. location mapping effectiveness).

B. Identify if any agreement among participants for bend direction exists, when prompted with the same set of parameters.
a. Intensity
b. Waveform
c. Duration
d. Motor combination

The following sections outline the guidelines used when designing the different Tactons, an overview of the Tactons used, hardware and software created to run the study, overview of the methodology, analysis and discussion.

\subsection{Tacton Design}

Although exploration and research into haptic stimulus and the design of Tactons spans a range of areas $[8,49]$, we are not aware of any work completed that use haptic stimulus to prompt bend gestures in the z-axis. Since this is the case, we focused on six 
basic navigation bend gestures that have proven to be successful location and interaction choices $[13,15,47]$ (Figure 4). These gestures include: top left corner bend up and down, top center bend up and down, top right corner bend up and, where up represents towards the participant.

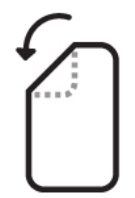

TOP LEFT DOWN

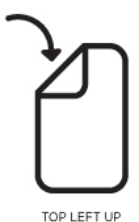

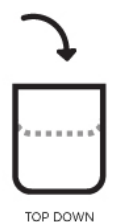

TOP DOWN

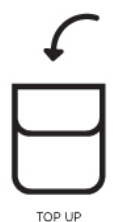

TOP UP

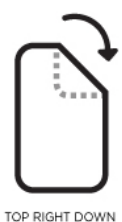

TOP RIGHT DOWN

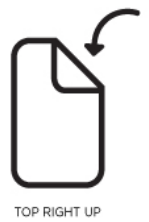

Figure 4 Six bend gestures used in study 1

The gestures used for bend were specific to a smartphone device in portrait mode, based on prior work $[29,47]$ and the wider adoption and saturation of the form factor within the general population.

\subsubsection{Parameter Definition}

Defining a set of Tactons that map to three bend gesture pairs and represent a consistent meaning across users could be a valuable contribution to the research of accessibility and deformable user interfaces. Within this objective, we faced the difficulty of determining what parameter could be associated to the z-axis (i.e. towards or away from the user). Yatani and Truong [49] successfully mapped vibrations to swipe gestures because they existed within the same plane. A vibration moving left to right is easily understood as a swipe from left to right. Unfortunately creating vibrations above or below the device is currently impossible with today's technology, so prompting the bending of corners up or down proved to be an interesting challenge. 
Based on the literature reviewed, a set of ground rules defined the Tacton design. Sahami et al. [40] identified that Tactons triggered at device corners had a higher accuracy of identification by users than middle areas. Yatani and Truong [49] found similar findings that spatial and directional Tactons has a 93\% accuracy of identification among their users. This provides solid evidence that we can use location as a parameter in our Tacton design when prompting bend gestures. Based on this work, we can assume that a vibration in the top left corner could be used to identify that that corner needs to be bent in certain direction.

Next, we explored a variety of parameters that could prompt a bend in the z-axis. Azenkot et al. [1] and Yatani and Truong [49] identify that Tactons containing patterns and location are preferred by users over a single stimulus. We explored different parameter combination options including rhythm, roughness and melody but settled on four options of intensity, waveform, motor combination and duration. We selected these parameters to explore what participants could perceive as a bend up or down. Changes in intensity or direction of the vibration felt on the device explored if participants could intuitively map them to a specific bend direction.

The intensity parameter posed an interesting exploration for the bend prompt. Based on Brown et al. [7] who found that users can identify an increase or decrease in intensity at $100 \%$ accuracy with either a linear or exponential progression, we explored if any agreement among participants would indicate a successful mapping of increase or decrease in vibration to bend direction. The objective of this parameter selection explored if a participant could mentally map the direction of the intensity wave up or down to a bend direction of up or down. 
We also explored the use of one and two motor combinations as an additional parameter. Two motor combinations used one of the top location motors (Left, Center, Right) and a central bottom motor. Using two motors paired with changes in intensity, we could simulate a directional pattern on the prototype. For example if the bottom motor started strong and faded to a weaker intensity while the top left motor started at a weaker intensity and ended strong it would simulate a vibration moving from the bottom of the device to the top left. We again wanted to explore if any agreement among participants would indicate a successful mapping to bend direction. This approach is similar to that taken by Yatani and Truong [49] when mapping touch gestures.

In addition to intensity and motor combination, we incorporated two different wave types, Square and Sine along with Tacton duration to understand if changes in intensity through different sensations of length would affect the participant's mapping or reaction time. This is based on work by Hoggan et al. [16] who identified that users could successfully distinguish between different waveforms (Sign, Sawtooth and Square) at a $100 \%$ accuracy rate and recommend any Tactons remain between 0.1 and 2 seconds in length, and differentiate by more than $10 \mathrm{~ms}$.

\subsubsection{Parameter Creation}

These four parameters, in combination with variation in Tacton location produced

48 Tactons in total. Each Tacton design used a basic formula of Tacton location + duration + intensity start to end + waveform + motor combination. Figure 5 illustrates this formula while Figure 6 illustrates a complete set of Tactons for a single location (Top Left). Table 1 provides an overview of all values used in this Tacton formula. 


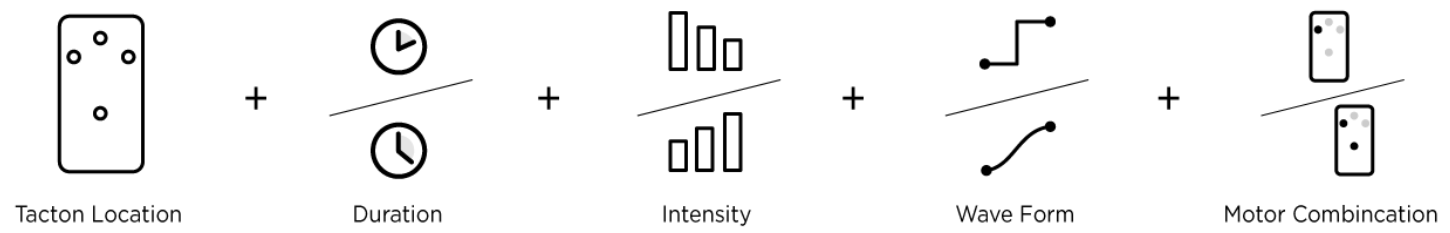

Figure 5 Illustration of the formula used to create each Tacton. Location + Duration + Intensity Start to End + Waveform + Motor Combination

We selected these Tactons from a predefined set of one hundred and twenty one Tactons that came packaged with hardware used to control the haptic motors [56]. We determined the duration of the Tactons by averaging four pilot participant's reaction time for every Tacton, Short durations were $0.45 \mathrm{~s}$, and long durations were $1.05 \mathrm{~s}$, with a standard deviation of $0.050 \mathrm{~s}$.

\begin{tabular}{|c|c|c|}
\hline $\begin{array}{l}\text { Motor (2) } \\
\text { - Single Motor (one of the } 3 \text { top locations) } \\
\text { - Dual Motor (one of the } 3 \text { top locations }+ \\
\text { the bottom location) }\end{array}$ & $\begin{array}{l}\text { Duration }(2) \\
\text { - Short }(\sim 0.450 \mathrm{~s}) \\
\text { - Long }(\sim 1.050 \mathrm{~s})\end{array}$ & $\begin{array}{l}\text { Waveform (2) } \\
\text { - Sine wave } \\
\text { - Square wave }\end{array}$ \\
\hline $\begin{array}{l}\text { Intensity }(2) \\
\text { - Low }(\sim 0.65 \mathrm{~g}) \rightarrow \operatorname{High}(\sim 1.4 \mathrm{~g}) \\
\text { - High }(\sim 1.4 \mathrm{~g}) \longrightarrow \text { Low }(\sim 0.65 \mathrm{~g})\end{array}$ & $\begin{array}{l}\text { Tacton Location (3) } \\
\text { top left } \\
\text { top center } \\
\text { top right }\end{array}$ & \\
\hline
\end{tabular}

Table 1 Breakdown of each parameter variables including: motor combination, duration, waveform, intensity and location. (g) is the amplitude measurement controlled by the voltage passed through the motor 

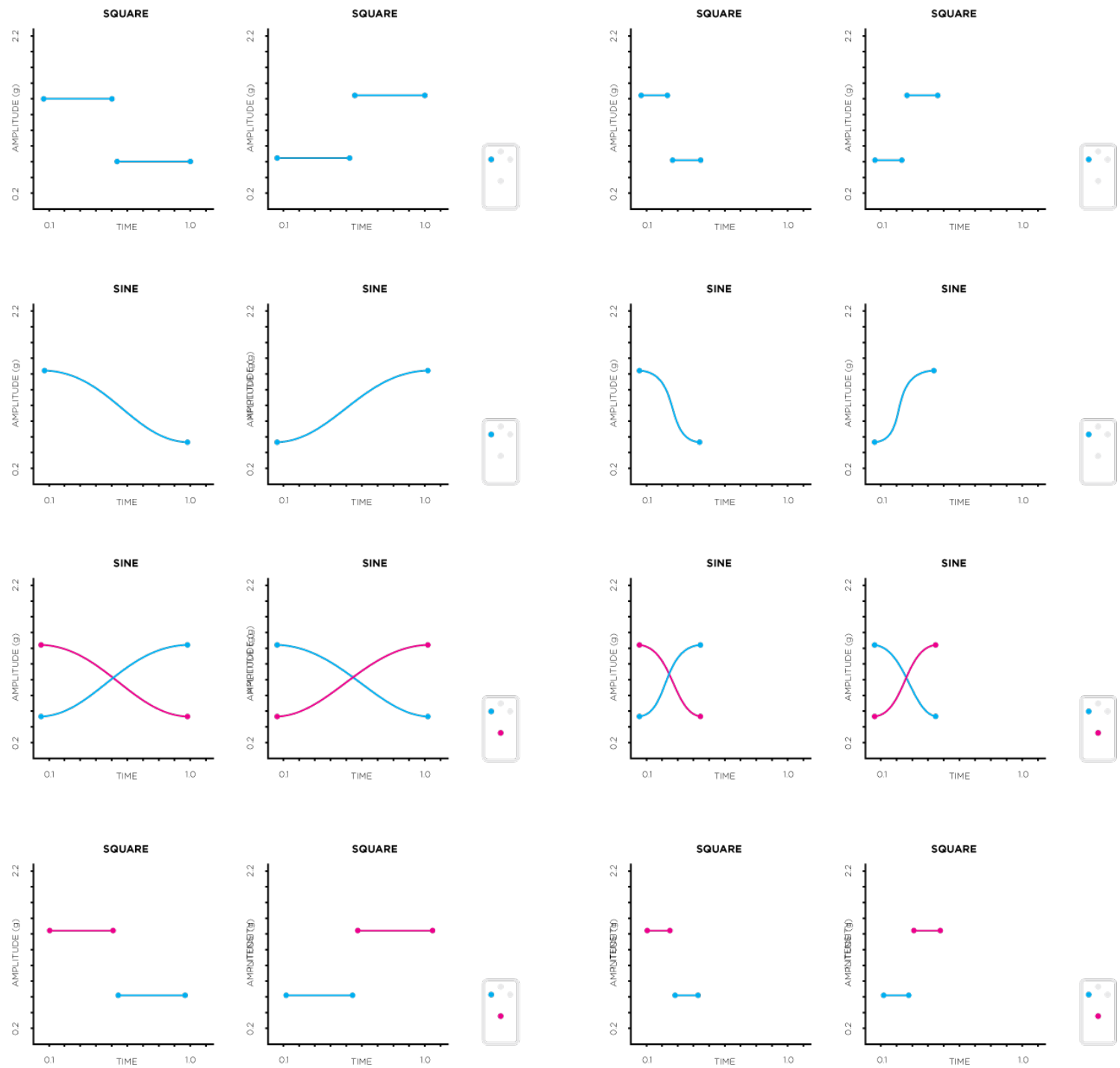

Figure 6 Simplified charts, visualizing a complete set of single Tactons for a single location (Top Left)

\subsection{Prototype Hardware and Software}

In order to deliver these forty eight Tactons to the participant, we designed a prototype that could effectively deliver the haptic stimulus and allow them to make a decision on what bend gesture they believe the system is prompting them to perform. The four main components included in Figure 7 include: 
1. Arduino Leonardo board and wiring, encased in a plastic housing.

2. Twelve digit keypad used by the experimenter to enter the Tacton ID number.

3. Handheld haptic prototype held by participants, but out of their line of sight.

4. Laptop used to input the participant's choice.

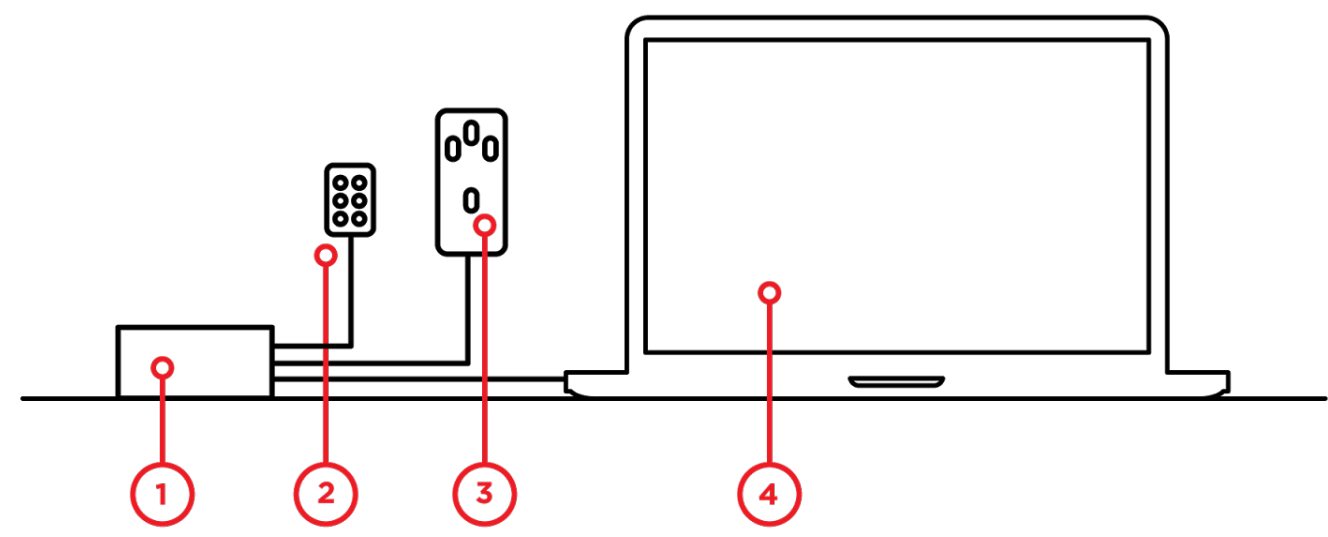

Figure 7 Study 1 prototype setup, including 1) Arduino Leonardo board, 2) 12 digit keypad, 3) Handheld prototype and 4) Laptop.

The overall system included Arduino components and software to control the prototype and present the Tactons to the participant and an HTML interface for them to enter their choice. For each task the experimenter entered a random number using the keypad that triggered a specific Tacton to fire.

The participant felt the vibration through the haptic prototype and made a selection on screen through the testing interface. Once a participant made their selection, a prompt asked the participant to rate their confidence in their decision.

The hardware used, included a Leonardo Aurdino board to run the processing, control the Tactons and send information to the HTML testing site through keyboard mapping. 


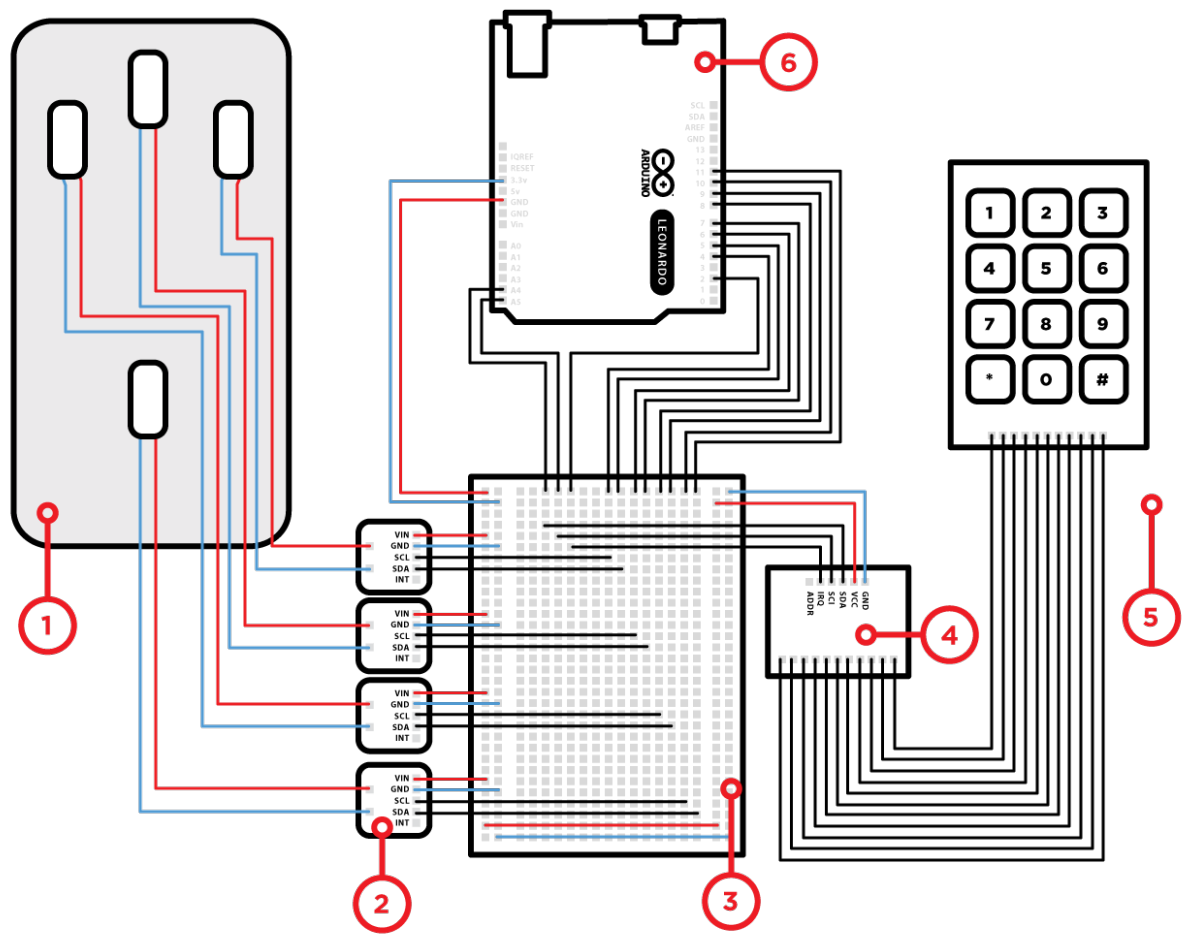

Figure 8 Capacitive Touch Keypad and Haptic Motor wiring. 1) cardboard prototype with 4 haptic motors, 2) Adafruit DRV2605L Haptic Motor Controllers, 3) Breadboard, 4) MPR121Touch Chip, 5) Keypad used to enter Tacton numbers, 6) Arduino Leonardo Board. Shaded area indicates the silicone prototype.

\subsubsection{Prototype Hardware}

Connected to the Leonardo board, the Capacitive Touch keypad by dfrobot [57] used a MPR121Touch Chip that allowed the experimenter to enter number values into the system and trigger the Tactons to fire. 4 Eccentric Rotating Mass vibration motors were controlled using Adafruit DRV2605L Haptic Motor Controllers [56]. These boards came with a pre-defined set of Tactons (121 in total), called using specific pieces of code. These controllers allow for a more refined control over the motors and instead of a basic on and off, we could control wave type, duration and motor combination, accommodating for all of the parameters earlier defined. Figure $\mathbf{8}$ illustrates the complete wiring of the prototype hardware. 
The third component in the system consisted of the hand held prototype. The approximate size of an iPhone $6(5.44$ " $\mathrm{H}$ x 2.64" W), it connected to the Leonardo using an 8 wire ribbon cable. Early versions of the prototype identified that to achieve maximum effectiveness of the vibration, the motors needed to be in close contact with the palm and fingers (Figure 9: a) illustrates the gap when using a flat prototype, also experienced by Yatani and Truong [49]. Our end result used a stiff cardboard that could be bent into a concave shape to form a closer bond to the participant's hand (Figure 9: b, c).
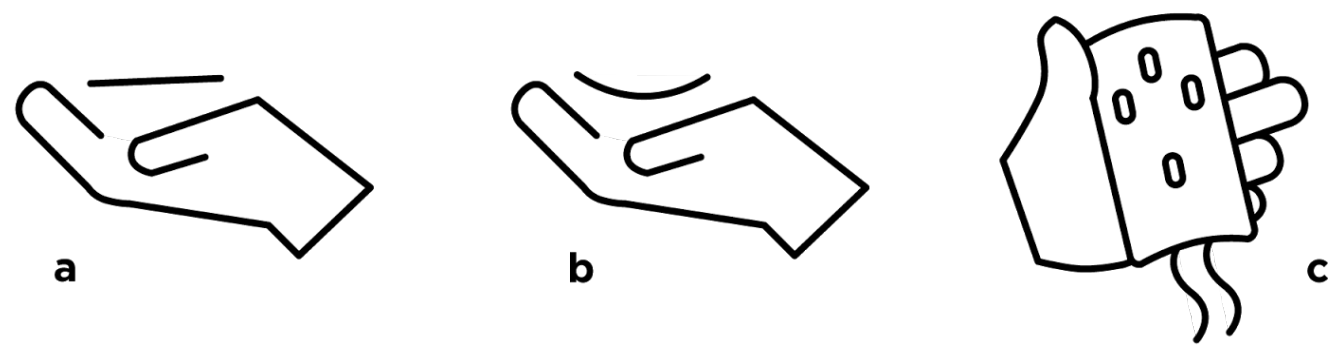

Figure 9 Illustrates the original flat prototype (a) that created space between the motors and palm. Further refinement of a concave shape $(b+c)$ maximized contact with the participant's hand and increased the identification of the vibrations.

During the early stages of the prototype development, we iteratively explored the configuration of 3 or 4 motors (Figure 10). We presented each motor configuration to a small group of individuals who were asked to identify which configuration presented the strongest vibration for each of the three locations. Certain 3 motor options were too confusing to these participants and caused miss identification of top center and top right locations. This occurred as participants experienced the top motors at different strengths. For example, prototype 3 (Figure 10) fired the left and right motors together to identify a top bend, which often resulted in a misidentification of top left among pilot participants. 
Prototype 4 reverted back to 4 motors with a central location to identify the top location. Although an improvement over the previous option, confusion among the top three motors still existed. Prototype 5 (Figure 10) illustrates the final solution and depicts the higher placement of the center motor. This created easily distinguishable areas for the participant to identify and resulted in a high accuracy rate during initial testing.

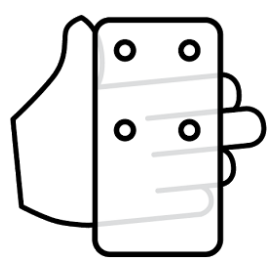

PROTOTYPE 1

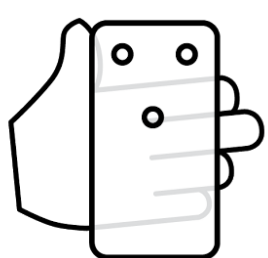

PROTOTYPE 2

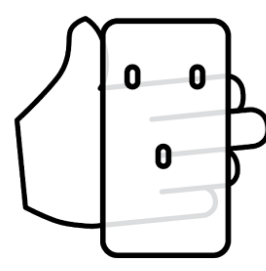

PROTOTYPE 3

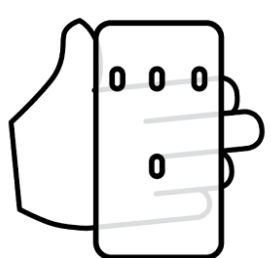

PROTOTYPE 4

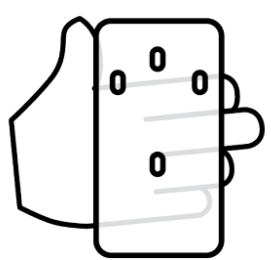

PRotoTres S

Figure 10 Evolution of motor combinations, placement and orientation

In addition to motor placement the orientation of the motors created variations in vibration spread. Earlier prototypes $(1+2)$ affixed the motors flat against the backing. This resulted in a more distributed vibration through the rest of the phone and a higher rate of misidentification at other locations. This is due to the rotation of the offset mass inside the motor that spins at a high speed to create the vibration. Positioned flat, it distributes the wave along the same plane as the device. Once the motors were turned vertically this wave disseminated in the z-axis and did not distribute as much vibration to the rest of the phone, resulting in a more localized stimulus. This approach differs from previous work $[4,20,49]$, and iPhone 5 or Android Samsung Galaxy S4 phones that orientate the motors horizontally, similar to our prototypes 1 and 2. 


\subsubsection{Prototype Software}

In addition to the hardware created to run the first study, the software consisted of two components: code written to run on the Leonardo to accept keypad number values and fire the Tactons ${ }^{1}$, and an HTML web app including the participant interface. The web app captured the keyboard events sent by the Leonardo and allowed the participant to enter their location and direction choices.

The web application enabled the participant to input their choice and capturing the data in a MYSQL database. The simple interface (Error! Reference source not found.Figure 11, Figure 12Error! Reference source not found.) visualized the three or six options available to the participant in relationship to an iconic representation of the device. Because the participant did not physically input their choice on a bendable phone, they utilized an onscreen UI with buttons indicting location and direction. Visual bend lines were included to assist the participant in better referencing location and bend direction and the blue dot to position the cursor between each task and create a uniform distance between options.

\footnotetext{
${ }^{1}$ Although the set up of a single controller was simple to accomplish, a contract developer from Fivr.com was paid $\$ 80$ to assist in the code development to run 4 simultaneously.
} 


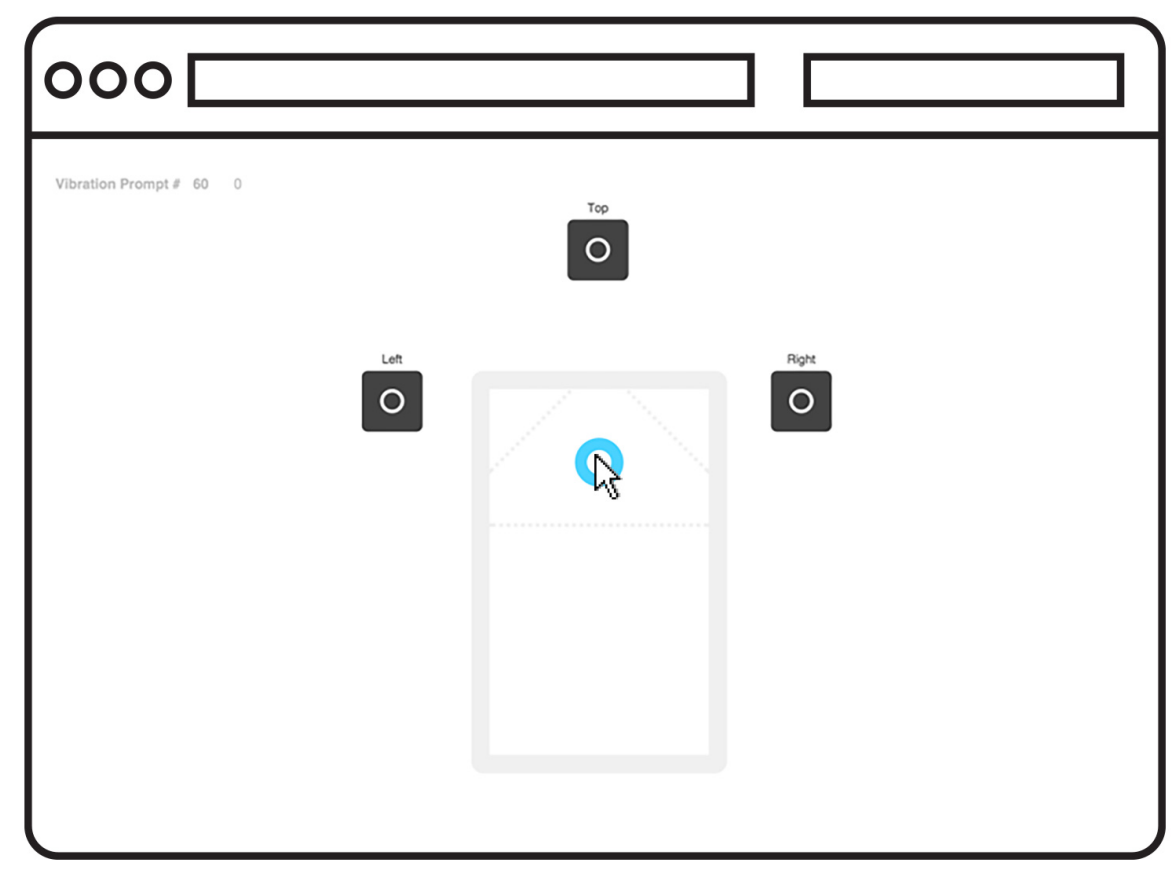

Figure 11 Web App: “Location” interface

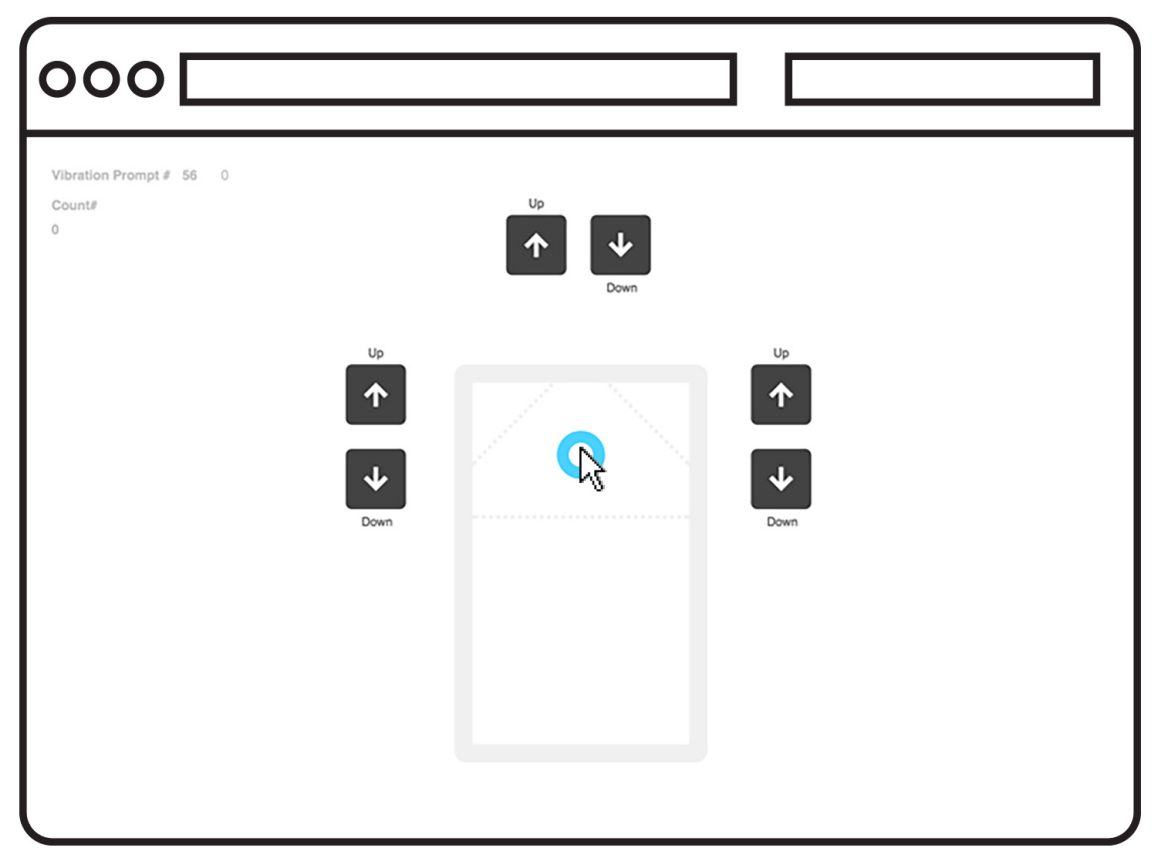

Figure 12 Web App: “Location/direction” interface 
The final setup is illustrated in Figure 13. Participants held the prototype off to the left side and out of sight during the study.
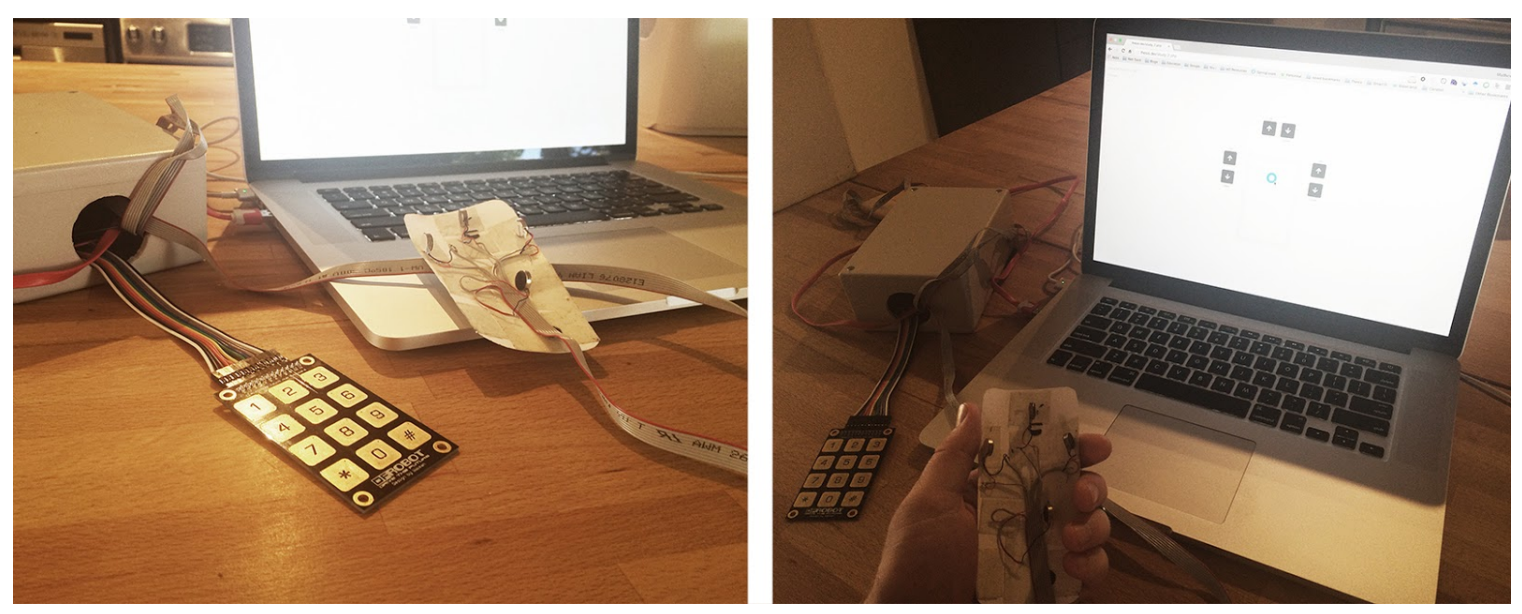

Figure 13 Final prototype setup from two angles.

\subsubsection{Methodology and Design}

Conducted in two stages, the 45 minute study explored if variations in location, intensity, duration, waveform and motor combination can effectively prompt a user to complete a bend gesture with no visual prompts. Previous work included a variety of methodological approaches such as Yatani and Truong [49] who blindly prompted users with a Tacton and then asked to input their option through on screen interface, or, Sahami et al. [40] who required participants to memorize the patterns ahead of time. Our approach followed a similar methodology to Yatani and Truong [49] and asked participants to choose which option they felt the vibration best represented.

We designed a $3 \times 2 \times 2 \times 2 \times 2$ repeated measures within-subject design, with the independent variables: Tacton (location, intensity, duration, waveform and motor combination). Dependent variables include: Bend direction (up and down) and location 
(top left, top center, and top right). The prototype would present a random Tacton to the user who would then be asked to map it to a bend location and direction. Each Tacton lasted half or one seconds, depending on the duration parameter, and repeated four times with a two second pause in-between. Repeating the Tactons automatically allowed the participants time to best identify which location and direction was being prompted.

Once the participant felt comfortable in their decision, they selected one of the six options on screen. After their decision, they rated their confidence on a 5 point Likert scale: (On a scale of 1 to 5 how confident are you with your choice?: 1 being not at all and 5 , very).

\subsubsection{Study Breakdown}

Each session included four components: introduction, training phase, study and post interview. Once introduced to the study, the prototype components and methodology, the participant completed a set of demographic and basic expertise questions. The preliminary demographic based questions included age and sex along with rating their experience with technology and bendable phones on a scale of 1 to 7 , with 1 being poor and 7 excellent.

Next, the researcher demonstrated the six bend gestures on a silicone phone cast designed to bend in the three key locations and two directions. The participant interacted with the cast only to become familiar with the three gesture pairs and center squeeze. Once the participant became comfortable with the concept of bend gestures, the testing proceeded with the first training session with them holding the haptic prototype in their left hand. The objective during this stage ensured the participant could correctly identify 
a location prompt when presented with six Tactons in total with the parameters: duration: long and short, and a single strength: strong. Participants did not proceed to the next stage until they had successfully identified locations with an accuracy of greater than $83 \%$ (five out of six Tactons). All participants successfully completed this stage on the first try. We also designed this step to ensure that all participants had a similar level of touch sensitivity within their palms and fingers. We utilized this simple method to screen for participants for any touch or physical issues that could skew results during the study.

The main section of the study consisted of the presentation of 48 Tactons in random order to the participant. With the goal to identify if any agreement among participants between bend direction and parameter, the participant felt a randomized Tacton and selected a location and the direction they felt it represented. During all testing, the participant positioned the haptic prototype out of the view plane between them and the laptop screen to simulate visual impairment.

\subsubsection{Data Collection}

At the end of the study, we asked participants to explain their process for deciding which direction to choose based on the specific vibration stimulus. We recorded each participant's preferred method for directional bend mapping, as well as confusion rating for using one or two motors. This rating used a 5 point Likert scale: (On a scale of 1 to 5 how confusing did you find one motor vibrations?: 1-Not at all to 5 - Very).

The quantitative data recorded for each task during study included: Unique ID number, participant number, task number, location and bend choice and participant's confidence. 


\subsection{Analysis and Discussion}

We gathered both qualitative and quantitative data during the study. The quantitative data included location and bend choice, task reaction time and participant's confidence with their decision. Video recordings, researcher observations and an informal interview captured qualitative data.

12 adult male and 5 adult female participants between the age of 21 and 44 with an average age of 31 completed all stages of testing. All participants identified as having normal vision with or without corrective lenses. The average technical proficiency for all participants was 5.1 based on the $7 \mathrm{pt}$ scale ( 1 being poor to 7 being excellent). 10 of the participants had limited experience with bend gestures as they were participants in study 2 while the remaining 7 had no experience at all. Participants were compensated $\$ 10$ for their time.

\subsubsection{Location Accuracy}

We first identified if changes in the parameters waveform, duration, motor combination and intensity affected the participant's accuracy when selecting a location. To isolate a participant's location choice, we combined their bend choice results for each location. During this study, $75.41 \%$ of users identified the location correctly across all variables. $80.75 \%$ identified the top left location correctly, $74.81 \%$ of participants identified the top center location correctly and $70.79 \%$ of participants identified the top right location correctly. Table 2 identifies these three combinations as agreement percentages.

We conducted a repeated measures analysis of variance (ANOVA) test for accuracy rate against each parameter and location. Out of all of the 48 possible computations, the motor combination factor had a statistically significant effect on 
location choice $(F(1,9)=9.893, p=.012)$, as well as the motor and duration parameter combination $(F(1,9)=15, p=.004)$. The combination of motor and location parameters also produced a close to significant effect. $(F(2,18)=4.027, p=.056)$.

For the motor factor, the single motor parameter led to a higher accuracy $(82.54 \%)$ than double motors $(67.82 \%)$. We ran a post-hoc, Bonferroni corrected estimated marginal means test for the interaction between motor and duration. We found a significant effect for the combination of single motor and shorter duration resulted in a $89.2 \%$ accuracy $(p=.045)$, as well as for the combination of the single motor and longer duration resulted in a $79.2 \%$ accuracy $(p=.036)$. Table 3 identifies these three combinations as agreement percentages.

\begin{tabular}{lccc}
\hline \multicolumn{1}{c}{ Motor Parameter + Location } & \multicolumn{3}{c}{ Location Recorded } \\
\hline Single Motor & Top Left & Top Center & Top Right \\
\hline Top Left & $\mathbf{9 1 . 9 1 \%}$ & $6.62 \%$ & $1.47 \%$ \\
Top Center & $10.37 \%$ & $\mathbf{7 5 . 5 6 \%}$ & $14.07 \%$ \\
Top Right & $3.68 \%$ & $16.18 \%$ & $\mathbf{8 0 . 1 5 \%}$ \\
\hline Double Motor & & & \\
\hline Top Left & $\mathbf{6 8 . 9 9 \%}$ & $18.60 \%$ & $12.40 \%$ \\
& & & $16.15 \%$ \\
Top Center & $10.00 \%$ & $\mathbf{7 3 . 8 5 \%}$ & $\mathbf{6 0 . 6 1 \%}$ \\
Top Right & $4.55 \%$ & $34.85 \%$ & \\
\hline
\end{tabular}

Table 2 The confusion matrix for the statistically significant parameter: motor number for each of the location prompts. The bold numbers represent the correct location choices made by the user.

Although the overall combination of motor and location produced a close to significant effect $(p=.056)$, when we examine the individual locations, we find that the 
combination of single motor and top left location result in a $91.91 \%$ accuracy with significant effect $(p=.028)$, and the combination of single motor and top right location result in a $80.15 \%$ accuracy with significant effect $(p=.049)$. Single motor and top center location did not produce any significance.

\begin{tabular}{lccc}
\hline \multicolumn{1}{c}{ Motor + Duration + Location } & \multicolumn{3}{c}{ Location Recorded } \\
\hline Single motor + Short & Top Left & Top Center & Top Right \\
\hline Top Left & $\mathbf{9 4 . 1 2 \%}$ & $4.41 \%$ & $1.47 \%$ \\
Top Center & $4.48 \%$ & $\mathbf{8 5 . 0 7 \%}$ & $10.45 \%$ \\
Top Right & $2.94 \%$ & $16.18 \%$ & $\mathbf{8 0 . 8 8 \%}$ \\
\hline Single motor + Long & & & \\
\hline Top Left & $\mathbf{8 9 . 7 1 \%}$ & $8.82 \%$ & $1.47 \%$ \\
Top Center & $16.18 \%$ & $\mathbf{6 6 . 1 8 \%}$ & $17.65 \%$ \\
Top Right & $4.41 \%$ & $16.18 \%$ & $\mathbf{7 9 . 4 1 \%}$ \\
\hline Double motor + Short & & & \\
\hline Top Left & $\mathbf{6 4 . 0 6 \%}$ & $23.44 \%$ & $12.50 \%$ \\
Top Center & $9.23 \%$ & $\mathbf{7 8 . 4 6 \%}$ & $12.31 \%$ \\
Top Right & $4.55 \%$ & $39.39 \%$ & $\mathbf{5 6 . 0 6 \%}$ \\
\hline Double motor + Long & & & \\
\hline Top Left & $\mathbf{7 3 . 8 5 \%}$ & $13.85 \%$ & $12.31 \%$ \\
Top Center & $10.77 \%$ & $\mathbf{6 9 . 2 3 \%}$ & $20.00 \%$ \\
Top Right & $4.55 \%$ & $30.30 \%$ & $\mathbf{6 5 . 1 5 \%}$ \\
\hline & & & \\
\hline
\end{tabular}

Table 3 The confusion matrix for the statistically significant parameter combination: motor number and duration for each of the location prompts. The bold numbers represent the correct location choices made by the user.

\subsubsection{Bend Direction}

We approached bend direction from the perspective of an unknown result, which allowed us to draw conclusions around the agreement among participants on the direction of bend 
when presented with a specific Tacton. We further identified if any individual or combination of parameters: waveform, duration, motor combination, intensity and location had significant effect on the agreement score.

\subsubsection{Bend Direction Association}

To identify if these Tactons and parameter combinations are significant, we conducted a Factorial Logistic Regression test between the direction of the gesture performed and the parameters. We found the pairing of an increasing intensity and two motor combination to be significant $(\mathrm{p}<.0005)$. The rest of the factors were not significant. Figure 14 illustrates the bend choice of participants when presented with this pairing of motor combination and intensity. Single motor Tactons clearly result in no consensus on bend direction but double motor Tactons do and with significant results. $77.78 \%$ of participants selected bending down with an increase in intensity while $65.78 \%$ of participants selected bending up with a decrease.

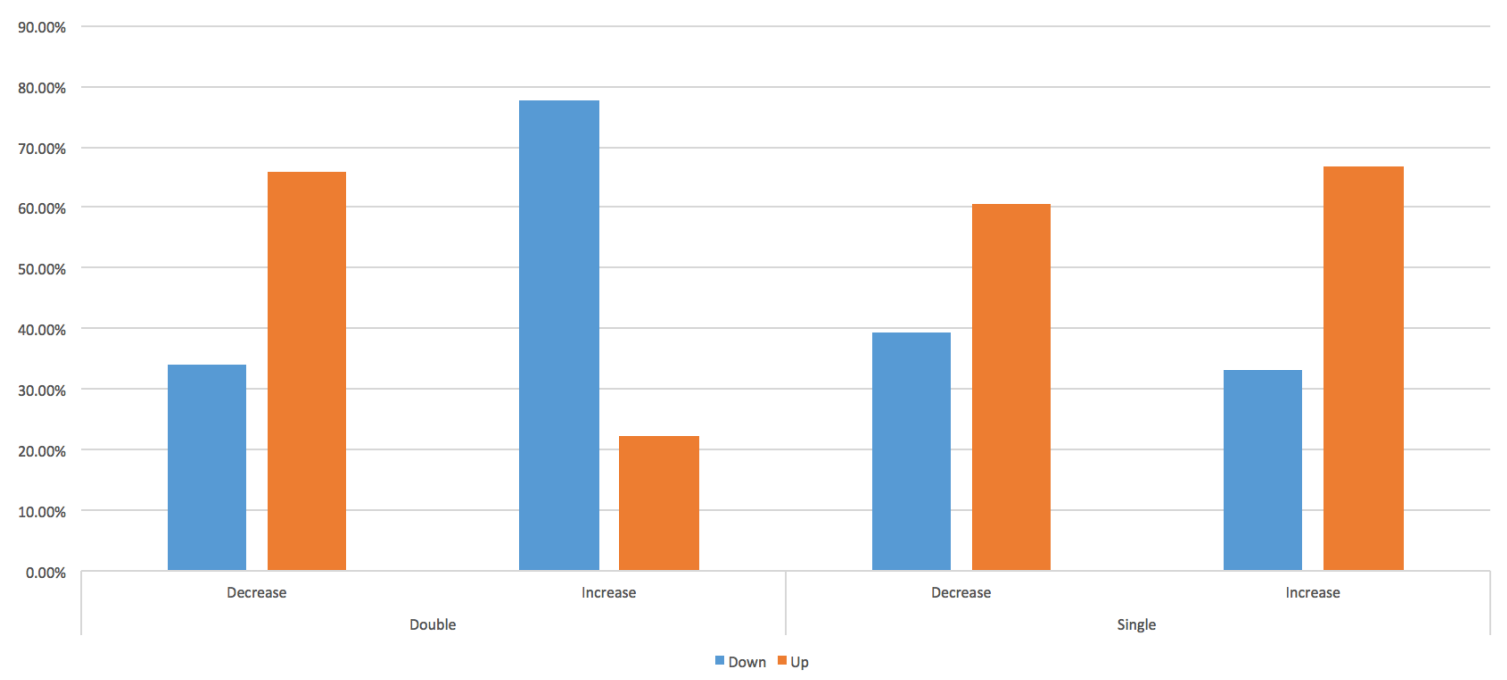

Figure 14 Illustrates participant's bend selection based on motor combination and intensity. 
When comparing a double motor Tacton set that could be used to prompt bend gestures, the use of a sine wave and short length in addition to two motor combination, resulted in $74 \%$ of participants selecting down with an increase in intensity while $70.59 \%$ of participants selected up with a decrease. The most successful Tacton using long, square wave with double motor resulted in $83.33 \%$ of participants selecting down with an increase while only $66.67 \%$ selected up with a decrease in intensity.

Figure 15 illustrates the mapping of these double motor and intensity parameters to the vibration feel when holding the prototype. For example: Double motor Tactons that fade from the bottom motor to the top is considered a decrease. Where a fade from a top motor to the bottom is considered an increase in intensity.
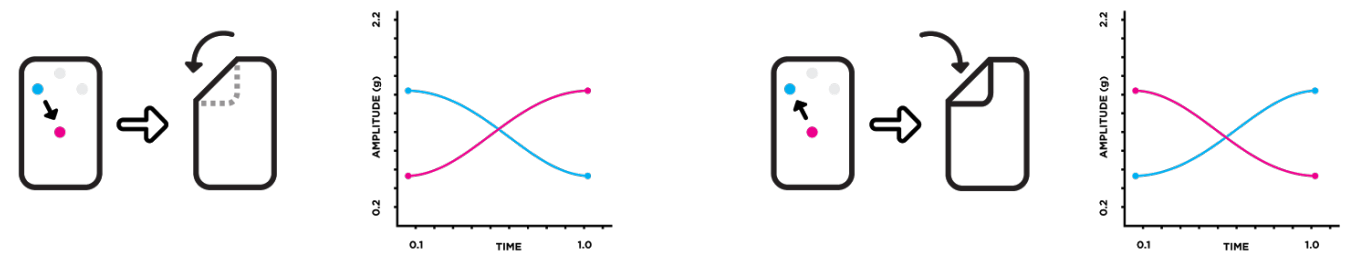

Figure 15 Mapping of participants Tacton feeling to bend direction agreement

\subsubsection{Mapping Confusion}

During the post study interview participants were asked to identify how confusing they felt the use of single or double motor Tactons were in prompting bend gestures. Participants rated their choice on a Likert Scale where 1 is not at all and 5 very. Figure 16 identifies the distribution of these responses. 


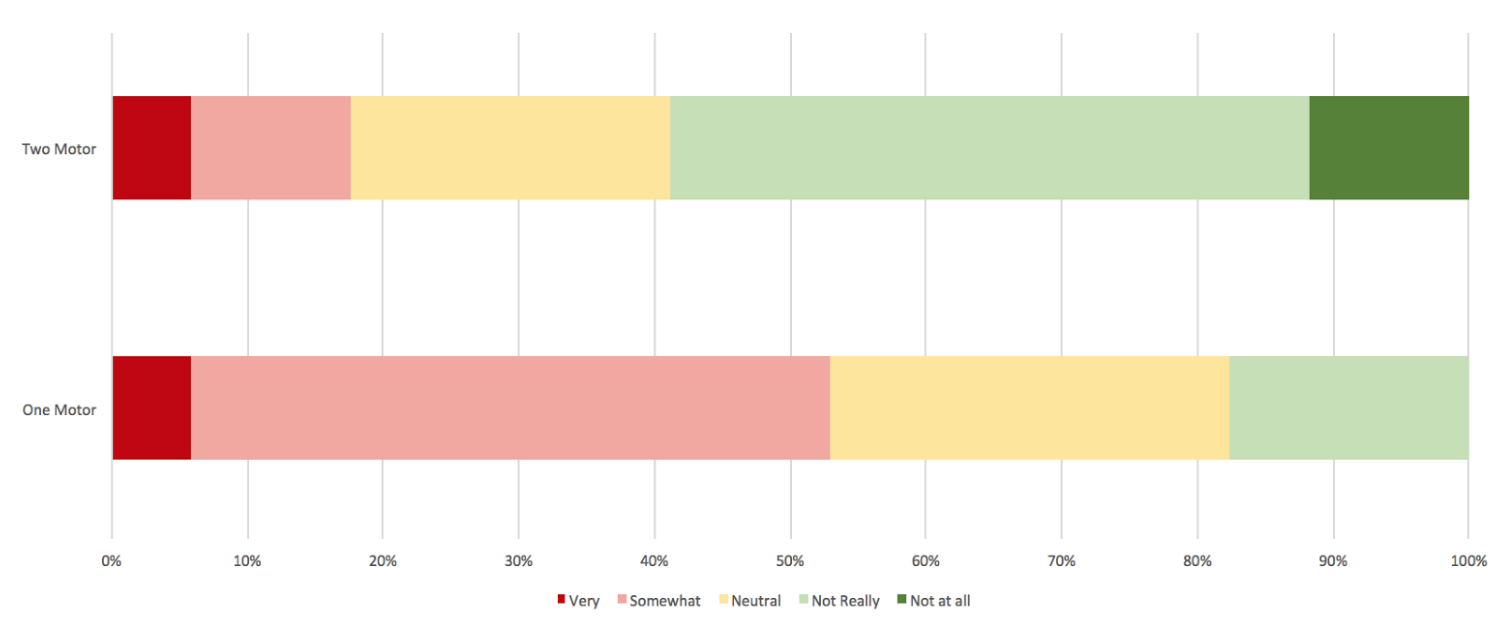

Figure 16 Distribution of Likert Scale responses for participants when asked to rate the confusion level for single and double motor Tactons.

Overall, participants found one motor Tactons more confusing than two. A Wilcoxon signed-rank test determined a close to significant result when comparing number of motors $(\mathrm{p}=0.06)$. During additional questioning, 4 participants identified "just guessing" at the single motor Tactons with respect to bend direction.

\subsubsection{Qualitative Findings}

In addition to the quantitative data identified above, we captured qualitative data in the form of an informal interview. Four participants identified the top vibration as harder to distinguish than other locations and felt it more muffled and traveling to other parts of the device. Three participants specifically commented on vibrations traversing left to right and right to left, which did not align with any Tacton presented to them. One participant explicitly mentioned creating a mental image or chart for up gesture going from soft to hard based on intensity. 


\subsection{Discussion}

We analyzed the data from the first study to gain insights into any agreement among participants with respect to prompting bend gestures with certain Tacton designs. We recorded participant's location and bend direction choice for each of the 48 Tactons along with task completion time. When compared to past work, we recorded a slightly better location accuracy rate. Sahami et al. [40] identified their participants picking the top left location with a $73 \%$ accuracy compared to ours at $80.75 \%$. They also identified a lower accuracy for the top right location at $63 \%$ compared to our participants at $70.79 \%$. It is interesting to note the almost identical $10 \%$ difference in accuracy across locations. This leads us to believe that although slightly higher, our results are consistent with past work and re-enforces the accuracy of location with the use of Tactons. The higher results for our study may have been attributed to the style of prototype used. Sahami et al. embedded the motors within a fake casing where our motors were positioned on top of a thick cardboard. Dampening may have occurred within their prototype which is something we experienced early on when embedding motors inside silicone. Even at 70 to $80 \%$ accuracy it still means 1 out of 5 users guessed incorrectly and improvements in the prototype, increased familiarity with the device and continued exposure to the Tactons would help to improve these numbers.

In addition to location accuracy, we calculated consistency for each of the Tactons with respect to participants' bend direction choice. This gesture selection among participants is lower than previous work completed using touch gestures [49] but significant differences did occur for a select few combinations. Within our study, the concept of bending in the z-axis is a more complex interaction to communicate using 
haptic stimulus than touch. Work completed by Yantani and Truong [49] prompted touch gestures using Tactons with an $89.6 \%$ accuracy rate across 11 patterns but the sensation of the vibration and the perceived touch gesture existed within the same $\mathrm{x}$ and $\mathrm{y}$ axis. Our study presented a vibration in the $\mathrm{x}$ and $\mathrm{y}$-axis but asked the participant to pick a direction in the z-axis. The original hypothesis explored if different parameters such as intensity could significantly prompt this directional choice using one motor, (eg. increasing from a weak to strong vibration indicates a bend toward the user).

$83 \%$ of participants identified the use of double motors as not confusing or neutral when prompting bend direction and is reflective of the significant number of participants (77.78\% ) picking bending down with an increase in intensity. Although only $66.67 \%$ of participants selected up with a decrease overall it jumps to $70.59 \%$ with a specific pairing of parameters. Figure 15 represents the direction of the vibration felt on the prototype mapping to the perceived direction to bend. For example if the participant felt the bottom motor increased in intensity while the top left motor decreased they mapped it to bend down.

The relationship of vibration perception to direction needs further investigation with a realistic prototype. Although significant results indicate the use of two motor combinations and intensity could prompt a bend direction, it is still unclear if participants truly mapped to bending in the z-axis or the on screen arrows within the interface.

The contrast between the accuracy of location with single motor Tactons and the agreement between participants around the use of two motors as an indicator to bend direction presents an interesting design challenge. Future work could explore the combination of a short single vibration in a corner to identify location followed by a two 
motor vibration to indicate direction. We also see further expansion of work completed by Hoggan et al. [16] and the use of Tactons to convey specific types of notification meanings in combination with bend direction prompts. For example, a vibration traveling from the top right to the bottom could indicate not only that the user has an incoming phone call but also the bend gesture required to answer the call. It would also be interesting if these vibrations could decrease reaction time and reduce errors among users in a longitudinal study where they are prompted to perform a previously learnt bend gesture set some time after being introduced to it. These qualitative findings add additional value for bend gestures and the use of haptics as non-visual prompts and communication. Improvements in vibration motor choice and construction techniques could lead to further improvements in agreement and accuracy. 


\section{Chapter: Study 2: Bend Vs. Touch Gestures}

With technology designed primarily for the sighted population, many visually impaired users confront accessibility and usability issues on a daily basis [30]. Solutions created to help the visually impaired interact more effectively with touch screen devices include: voice interaction, haptic vibration [49] and audio feedback [50,51,53,54] One solution not yet explored is the use of bend gestures as a valid form of input for visually impaired users. Successfully proven as a comparable form of input for a range of form factors and device sizes, [15,27,41,45], bend gestures innately require physical manipulation of a device to interact with an interface. This tactile form of input has potential for a demographic that relies heavily on touch to understand and interact with the world around them.

Previous DUI studies focused mainly on the use of projection based techniques or flexible screens to provide the user with visual feedback $[17,27,41,48]$. Non-visual feedback, such as audio or haptic, in conjunction with bend gestures can benefit all users when screen occlusion occurs and has yet to be explored. Being able to interact with a device without visually seeing it can exist in numerous everyday scenarios, such as driving a car, walking or holding the phone in a winter jacket with non haptic gloves.

\subsection{Goals and Objectives}

This study aims to explore if a visually impaired user can perform common tasks using bend gestures with audio feedback, compared to performing the same tasks using touch gestures. We compared the task completion time of participants completing a series of tasks using both bend and touch gestures, and evaluated which gesture type performed 
best within a visually impaired environment. Task completion time provided a base comparison when comparing the two, and allowed us to draw general conclusions on usability and efficiency based on the results. For example, if participants could perform basic navigation faster using bend than touch, we can interpret it is an indication that bend may be more efficient.

We also explore qualitative findings around users preference for interaction techniques, hand position, movement, and a user's perceived understanding of the interface. Because the screen is fully occluded from the participant, a mental understanding of the interface is needed while interacting with the device. We explore if bend gestures could better aid in the creation of this model when compared to touch gestures.

We predict that due to the screen occlusion, and the user's sole reliance on nonvisual feedback in the form of tactile cues and audio, bend gestures will be a preferred method of interaction. This is also reflective of past work identifying users preference for a more tactile method of interaction $[25,48]$.

The following sections outline the guidelines and process used when designing the different bend gestures, hardware and software created to run the study, an overview of the methodology, analysis of the data and further discussion.

\subsection{Task, Gesture Classification}

For this comparative study between bend and touch gestures within the VoiceOver system, we developed a set of bend interactions that would allow the user to perform common tasks such as finding a song to play, moving an email to the junk folder and changing the VoiceOver Rotor setting. 
The basics of VoiceOver are simple: there is always an on screen focus, and, as you navigate around the screen, the device will read aloud the focused item. Navigation within VoiceOver uses tabbing to move to the next or previous item on the page in a systematic order. Performing a touch gesture such as swipe right will shift the focus to the next item, which is then read aloud.

VoiceOver also has a rotor setting that allows the user to perform a series of additional actions in addition to navigation such as adjusting speech rate, ways of navigation, spelling out words. It allows the user to quickly change these different options with a rotation of two fingers in a clockwise or counter clockwise manner. For example, if the user is currently in the mail app, they can swipe left or right to navigate or swipe up and down to switch between different options that can be performed, such as "move to junk", "archive" or "flag". The user can then switch the rotor setting to another option, such as speech rate, and the same swipe up/down gesture now increases and decreases the rate at which the system reads allowed the on screen text. Although VoiceOver has a variety of different additional functions and settings, we designed the tasks in this study to focus on basic user interactions such as navigation, selection or adjusting the rotor setting.

We evaluated the different gestures and interactions performed by both touch and bend with nine tasks organized into three sets: navigation, action and rotor setting. Each task set composed of three tasks with similar goals and requirements. For example, the navigation set includes navigating the same playlist to find three separate songs. This approach allowed us to capture three navigation data points for each participant. The gestures used for bend and touch, are specific to a smartphone device in portrait mode. 
This size and orientation is based on prior work $[13,29]$ along with wider adoption and saturation within the general population [58].

\subsubsection{Swipe Gesture Classification}

To interact with the touch prototype, we adopt the predefined touch gestures established in the VoiceOver software to ensure consistency with the real world application. These include swipe left, right, up, down and rotate with two fingers clockwise and counter clockwise.Figure 17 illustrates the seven gestures that allowed the user to perform all tasks assigned during the study.

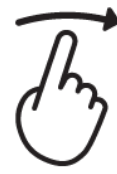

SWIPE RIGHT

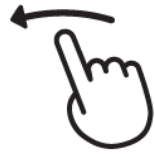

SWIPE LEFT

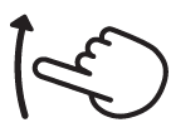

SWIPE UP

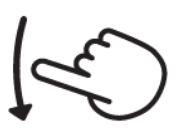

SWIPE DOWN

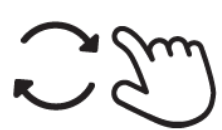

ROTATE CLOCKWISE/
COUNTERCLOCKWISE

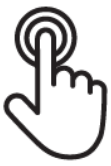

DOUBLE TAP

Figure 17 Seven touch gestures performed by participants

These VoiceOver touch gestures are independent of the user's hand dominance, which means swiping right or left with either the left or right hand would result in the same response from the system and navigate to the next or previous item in the list respectively. These interactions to gesture mappings are illustrated in Table 4. 


\begin{tabular}{l|l}
\hline Navigate to next selected item & $\begin{array}{l}\text { Swipe Right } \\
\text { Swipe Left } \\
\text { Navigate to previous selected item }\end{array}$ \\
Navigate to next rotator option & $\begin{array}{l}\text { Swipe Up } \\
\text { Swipe Down } \\
\text { Navigate to previous rotator option }\end{array}$ \\
Selecting focused item & $\begin{array}{l}\text { Double Tap } \\
\text { Two Finger Clockwise rotate } \\
\text { Switch to next rotor setting }\end{array}$ \\
\hline
\end{tabular}

Table 4 Complete breakdown of system behaviour and user gestures.

\subsubsection{Bend Gesture Classification}

In addition to the predefined VoiceOver touch gestures adopted into this study, a set of bend interactions defined prior to the experiment by a small set of HCI professionals established a consistent baseline. Based on prior work $[13,15,47]$ a general set of recommendations guided the creation of the bend gesture set for a portrait smartphone.

These recommendations include; utilizing the preferred interaction locations of the top two corners, top half of the device and squeezing. The primary interaction location should employ the corner of the device closest to the thumb. It is interesting to note that this creates a interaction set that is dependent on a user's dominant hand, whereas, touch is independent of the user's dominant hand, e.g. flicking right with a user's right hand performs the same interaction as if the user flicks right with their left hand. 


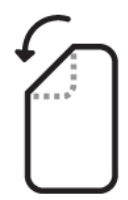

TOP LEFT DOWN

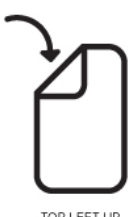

TOP LEFT UP

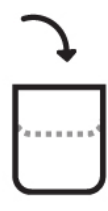

TOP DOWN

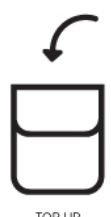

TOP UP

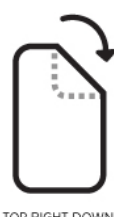

TOP RIGHT DOWN
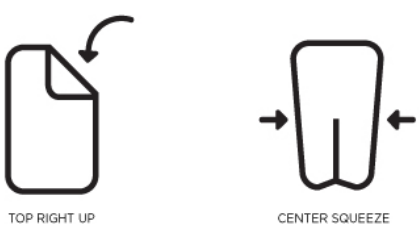

Figure 18 Seven bend gestures performed by participants

These findings resulted in a set of three bend gesture pairs: top left corner up and down, top right corner up and down, top up and down, and a single action center squeeze, illustrated in Figure 18. The corner closest to the thumb maps to navigation due to the most repetitive use, the secondary corner maps to action switching and the center squeeze maps to selection. Rotor switching maps to bending the top up or down. The center squeeze creates an interaction separate from overall navigation and therefore could not be confused or miss fired during other gestures. These interactions to gesture mappings are illustrated in Table 5.

\begin{tabular}{l|l|l}
\hline System Behaviour & Right Handed Gesture & Left Handed Gesture \\
\hline Navigate to next selected item & Top Right Down & Top Left Down \\
Navigate to previous selected item & Top Right Up & Top Left Up \\
Navigate to next rotator option & Top Left Down & Top Right Down \\
Navigate to previous rotator option & Top Left Up & Top Right Up \\
Selecting focused item & Squeeze & Squeeze \\
Switch to next rotor setting & Top Down & Top Down \\
Switch to previous rotor setting & Top Up & Top Up \\
\hline
\end{tabular}

Table 5 Complete breakdown of system behaviour and user gestures. 


\subsection{Hardware and Software}

To complete this study, we developed two prototypes that included a capacitive device to accept touch gestures, and a flexible silicone based unit with embedded bend sensors to accept the bend interactions. These prototypes allowed the users to perform bend and touch gestures to complete the defined tasks, interact with the main system and navigate the interface. In addition to this hardware, we created an interface that allowed the user to receive audio feedback as they navigated through lists.

The overall objective was to replicate the experience a user would encounter when using VoiceOver on any iOS smartphone device. This included ways to successfully navigate certain application interfaces, adjust settings and make selections while being provided instantaneous audio feedback.

The conscious decision not to use an actual iOS device and VoiceOver ensured a more consistent and comparable experience between both touch and bend. The low fidelity of the flexible prototype, when compared to an iPhone, would create a disadvantage from a speed, accuracy and user experience perspective when comparing results. Creating a prototype for touch interactions also allowed the user to receive the same audio feedback when interacting with the system, maintaining consistency.

Similar to the first study, the overall prototype system included 4 components as illustrated in Figure 19. 1) An Arduino Leonardo board and hardware encased in a plastic housing, 2) iPhone 6 sized bendable prototype, 3) iPhone 6 sized touch prototype 4) a laptop used to provide audio feedback to the user. 


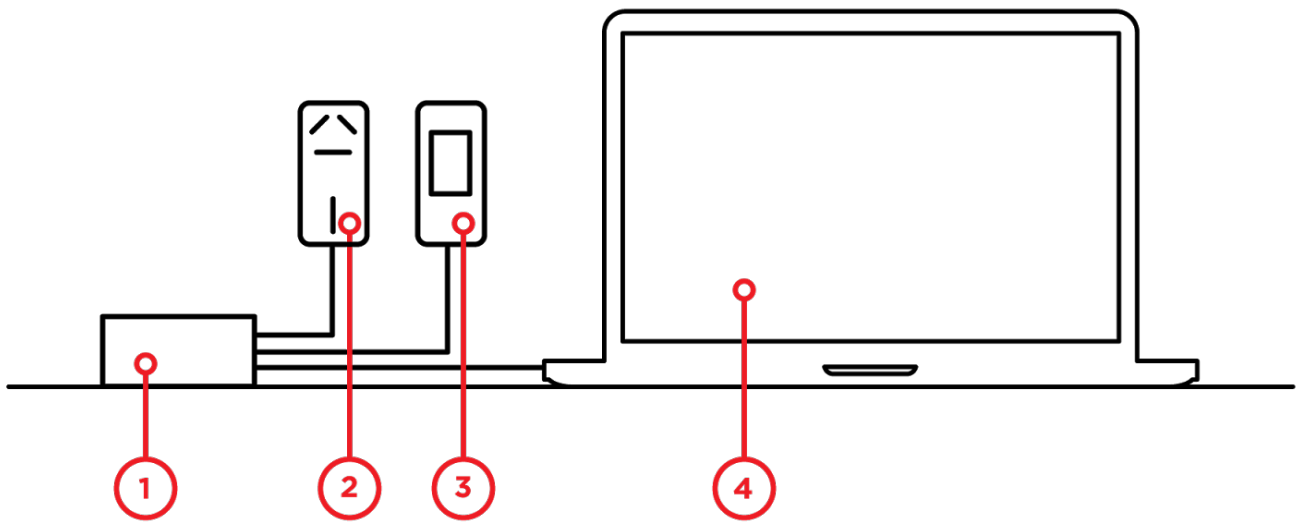

Figure 19 Overview of the 4 prototype setup components. 1) Arduino Leonardo board 2) Bend Prototype 3) Touch Prototype 4) System providing Audio Feedback.

\subsubsection{Prototype Hardware}

Similar to the first study, the Leonardo board received input from both the touch and bend prototypes and sent information to the HTML app through keyboard mapping. Connected to the board, the dfrobot capacitive touchpad [57] used a MPR121Touch Chip that allowed the user to perform basic touch gestures. This tracking the user's finger location and movement through the $\mathrm{x}$ and $\mathrm{y}$ coordinates and determined if the user swiped up, down, left or right. Bend sensors in the second prototype identified which location and direction the user bent which again mapped to the keyboard. Figure 20 illustrates the wiring of both prototypes. 


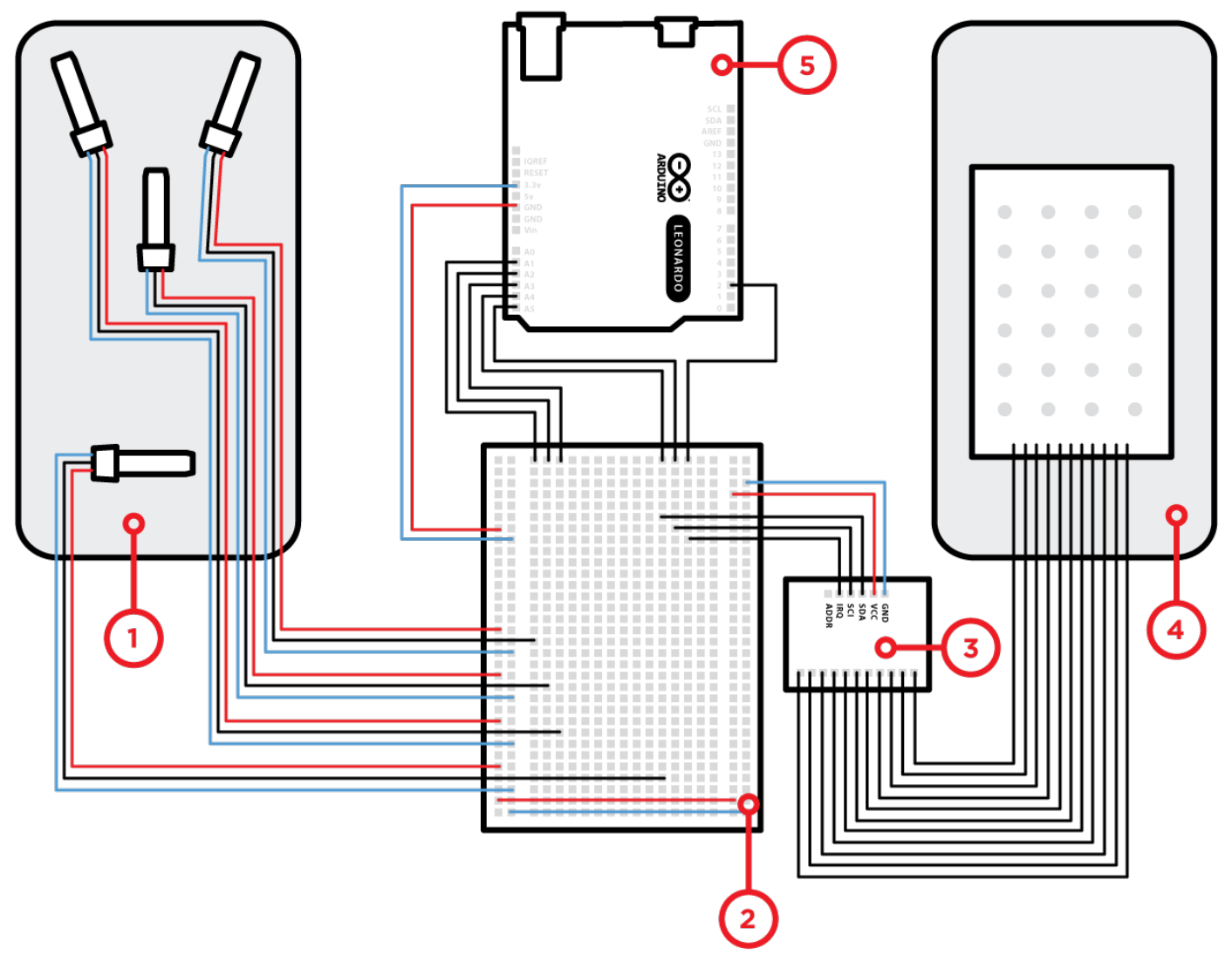

Figure 20 Capacitive Touch Pad and Bend Prototype. 1) Bend prototype with 4 bend sensors, 2) Breadboard, 3) MPR121Touch Chip, 4) Touch Prototype with capacitive pad 5) Keypad used to enter Tacton numbers, 6) Arduino Leonardo Board. Shaded area indicates the silicone prototypes.

Both prototypes used a silicone resin and were constructed in two stages. Based on prior work, [26,32] the prototype casting produced an object similar in size to a iPhone 6 a (120mm x 72mm).

The touch prototype included a base mold of similar size and thickness to the bend prototype with the capacitive touch pad affixed to one side. This created a consistent size, shape and weight between the two prototypes when held in the user's hand. The prototype connected to the Leonardo using 10-strand ribbon wiring.

Four bend sensors embedded within the silicone recorded the prototype's deformations. The prototype connected to the microprocessor using 12-strand ribbon 
wiring. Two bend sensors positioned at a 20-degree angle outward to each top corner captured the corner bend up and down. A third, positioned facing up in the middle of the prototype captured the top bend up and down and a fourth, positioned horizontally along the bottom captured the center squeeze in either direction. Figure 20 illustrates sensor placement.

\subsubsection{Improving Bendability with Stress Joints}

In order to better define the bend gestures for the second prototype, grooves embedded into the mold during the casting phase allowed participants to consistently trigger a defined bend with a lower error rate. Version 11 (Figure 22) illustrates the location of these grooves. This process created thinner depths at specific locations within the prototype, creating more flexible joints and allowing more defined and easier bends. Industrial design for "strain relief" used in today's power and computer cables, influenced the concept of stress points along the bendable joints. Designed to allow the cords to flex and bend without putting stress and strain on the cable itself, it incorporates a series of ridges along the ends of the cord as Figure 21 illustrates.

Figure 21 Images of strain relief designs incorporated into cables.

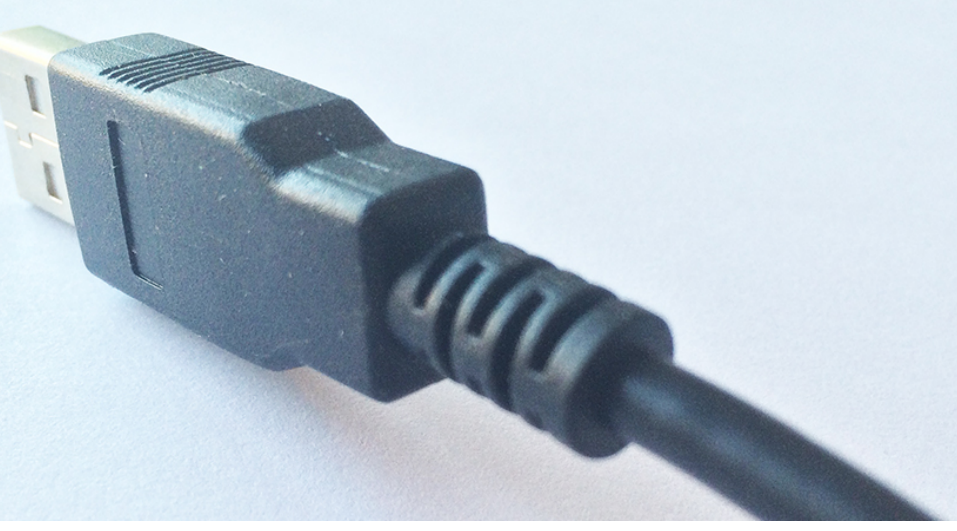


During the prototyping stage, we tested several versions, iterating variations in groove location, width and depth. Piloted with a small group of individuals, qualitative feedback allowed for refinements and iterations of the design. We presented each grove configuration to a small group of individuals who were asked to identify which prototype produced the most defined bend and with reduced errors.

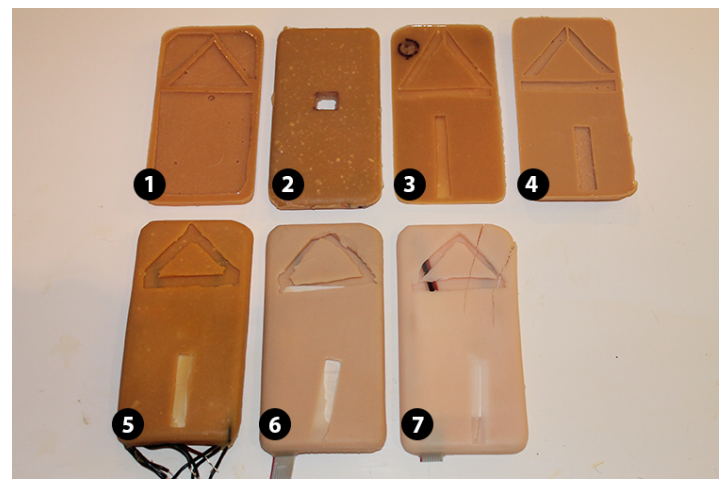

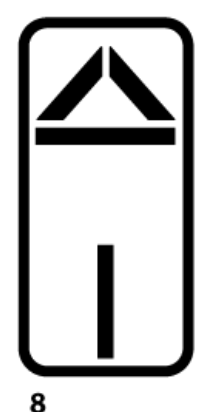

8

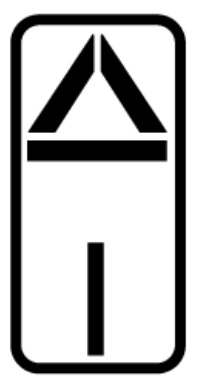

9

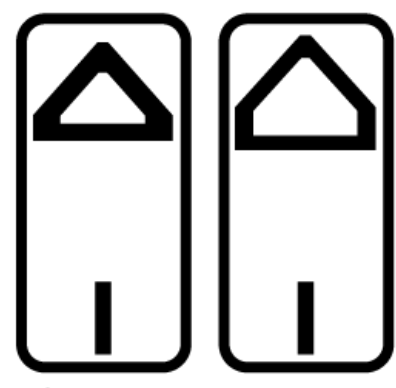

11

Figure 22 Construction of various prototypes testing groove location, depth and material density.

Version 1 (Figure 22) explored raised grooves instead of embedded, which quickly resulted in poor performance. Version 2 explored the combination of harder material (80A silicon resin) for the majority of the cast with the joints using 60A silicon resin. This produced no difference in flexibility compared with a cast of single consistent resin viscosity. Version 3 explored the use of negative space at the joints. This resulted in much better performance with clear bends along the joints. Versions 3 and 4 continued to explore different triangle shapes with separate pieces used to create the negative cavities. Although these resulted in improved performance over past versions they were positioned too close to the edges and resulted in misfires when performing other bend gestures. 
Versions 5, 6 and ultimately 7 resulted in preferred results from pilot testers and used a single triangle shaped mold to create the cavity.

Illustrations 8 to 11 visualize the molds used to create the various cavities along the joints. Version 11 resulted in the preferred performance with a balance of defined joint bend, device rigidity and comfort. The thickness of the top left and right segments were reduced in width and moved slightly closer to the corners. The bottom segment was moved further down the device and reduced in width.

\subsubsection{Prototype Software}

In addition to the hardware created to run the study, the software consisted of two components: Arduino code run on the Leonardo board, to capture both touch and bend gestures and an HTML web app simulated the VoiceOver environment. The HTML app captured the keyboard events sent by the Leonardo and provided feedback to the user through a digital audio voice as they navigated the interface.

Utilizing HTML and JavaScript (JS) a simple set of lists allowed the user to navigate an email and music app to complete the series of tasks. As the user navigates using either prototype, an Open Source JS library by Alon Zakai called Speak.js converted the in focus list element to audio output.

\subsection{Methodology and Study Design}

The design of the second study supported the overall objective of comparing the effectiveness of bend and touch interactions with full screen occlusion while receiving audio feedback. The methodology chosen required the participant to perform a series of tasks with both interaction techniques. 
We designed a $2 \times 3$ repeated measures within-subject design study, with the factors: interaction (bend, touch) and task type (navigation, action, setting), counterbalanced by interaction and randomized by task. Conducted in 3 stages and lasting approximately $45 \mathrm{~min}$, participants performed a total of 9 tasks representing the 3 task sets with each of the two prototypes. Our evaluation criteria consisted of task completion time for each of the nine tasks from the task starting to when the participant completed the final gesture, as well as overall preference for gesture type, comfort level for each task type and perceived understanding of the interface model.

\subsubsection{Study Breakdown}

Each session included three components: introduction, study and post questionnaire. Once introduced to the study, the prototype components and methodology, the participant completed a set of demographic and basic expertise questions. This captured participants' age, sex, dominant hand, technical, bend and VoiceOver experience. The last three data points were captured on a scale from 1 to 7 (1 being poor and 7 being excellent).

Participants first received an introduction to the interaction method (bend or touch) through a scripted demonstration, illustrating how to navigate a list, change actions, select a focused item and switch the rotor setting. A practice period allowed participants to try each interaction and listen to the audio feedback provided by the system based on the gesture performed.

Once participants were comfortable with the prototype, the researcher read aloud the task, and the participant performed a squeeze gesture to trigger the start of the task. This allowed the participant to control the start of the task and ensure they fully 
understood all requirements before proceeding. Once each participant completed the interaction type, they answered a short questionnaire,

Counterbalanced with each participant, the study portion required users to perform bend and touch gestures to navigate the interface based on a randomized list of tasks provided by the researcher.

\subsubsection{Task Overview}

We tested three core interaction paradigms within this study that included: Navigating up and down a list, performing additional actions on a focused item, and changing the function of secondary actions. The 9 tasks included:

1. NAVIGATION: Find the song "Sailors Eyes" and start playing it

2. NAVIGATION: Find the song "Wishful Thinking" and start playing it

3. NAVIGATION: Find the song "Cut Your Hair" and start playing it

4. ACTION: Find and forward an email from TicketMaster to your friend

5. ACTION: Find and flag an email you recently received from Porter Airlines

6. ACTION: Find the email from Paypal and move it to your junk folder

7. ROTOR: Change the VoiceOver Rotor Settings from "heading" to "links".

8. ROTOR: Change the VoiceOver Rotor Settings from "headings" to "speech rate".

9. ROTOR: Change the VoiceOver Rotor Settings from "characters" to "sections".

A full break down of tasks is attached in Appendix B. 

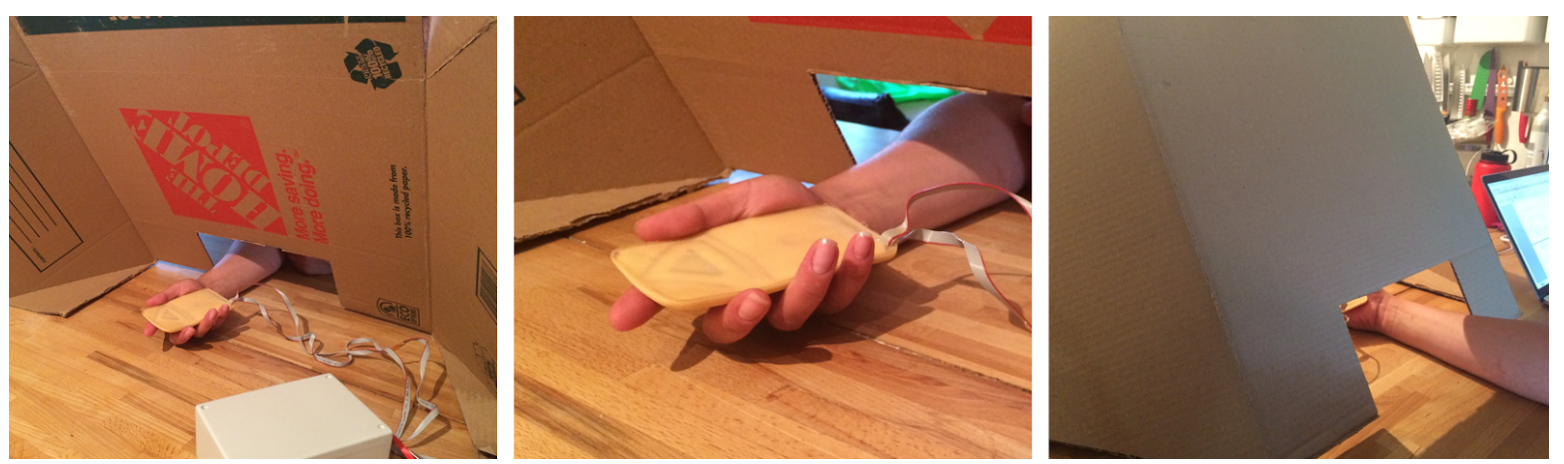

Figure 23 The screen and setup to create the visually impaired environment.

Figure 23 illustrates the screen placed between the prototype and the participant during both the practice period and task completion. Because we tested participants with no vision problems, this approach simulated the visually impaired experience using technology. It is comparable to that used by Yatani and Truong where participants held the device under the table [49]. This allowed the participant to interact with the device in a non-blindfolded and safer manner, but still maintaining unrestricted movement of the hands and forearms.

\subsubsection{Data Collection}

Data recorded during each session included participant number, task number, start time (initiated when the participant squeezed the device), end time (initiated when participant completed the appropriate action), gesture type (bend or touch) and hand dominance (left, right). Video capturing ensured all components of the study were video recorded, including the study control interface and participant actions with the prototype. 


\subsection{Analysis and Summary}

We gathered both quantitative data in the form of task duration, and qualitative data with participant hand movement and general observations. 17 participants completed 9 tasks for a total of 153 data points. Participants were 10 adult males and 7 females between the age of 22 and 39 with an average age of 29. All participants identified as having normal vision with or without corrective lenses. 16 participants were right handed and one left. 2 additional participants were unable to complete the testing phase due to prototype issues. The average technical proficiency for all participants was 5.5 based on the $7 \mathrm{pt}$ scale ( 1 being poor to 7 being excellent) and none of the participants had any previous experience with bend gestures at the beginning of this study. All participants had previous experience with touch gestures and using a smartphone device. Experience with VoiceOver was mixed: 8 participants rated themselves as a 1,4 participants rated as 2, 2 participants rated as a 3 and 2 participants rated as a 4 based on the same $7 p t$ scale. Participants were compensated $\$ 10$ for their time.

\subsubsection{Task Completion Time}

Analyzing the recorded quantitative data, we compared the average completion time across each of the 3 task sets and ran a two-way within-subject repeated measures ANOVA with factors: gesture type (bend and touch) and task set (action, navigation and rotation) against task completion time. We concluded that, no significant difference existed between completion times for each of the task sets, even though touch outperformed bend for the navigation, secondary actions and rotor setting adjustment tasks (Figure 24). 


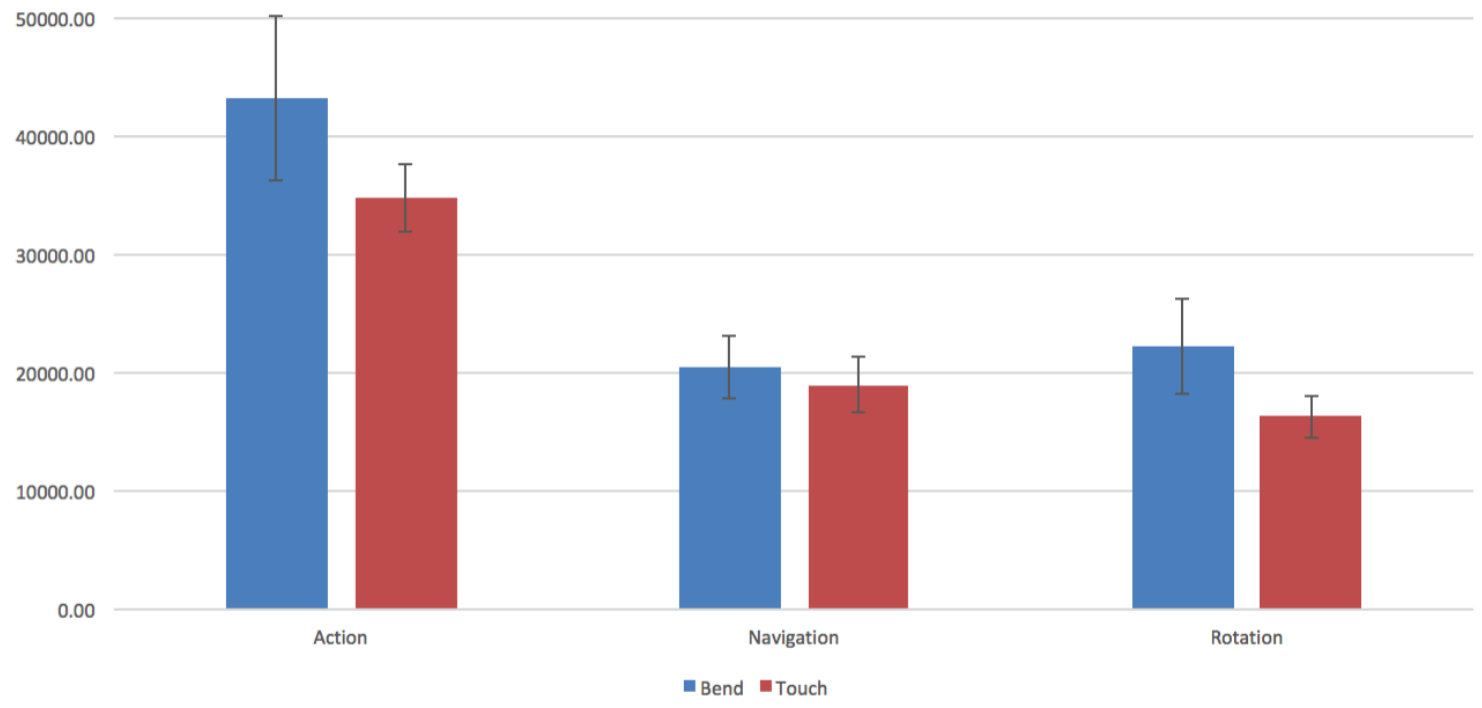

Figure 24 Average bend and touch completion times (milliseconds) for each of the 3 task sets including Standard Error bars.

\subsubsection{Re-gripping During Tasks}

Re-gripping during the bend interactions could have been a factor for the time differential illustrated in Figure 24. For action and rotation task sets, participants needed to switch from performing basic navigation gestures to a secondary gesture. For example, if the task required a right handed participant to delete a specific email using bend gestures, they navigated down the list of emails using the top left corner bending up and down, then, when that email was in focus, they switched between the different actions that could be performed using the top right corner bending up and down. Once the action was in focus, the user would squeeze the device to complete the task. Figure 25 illustrates this user flow. 


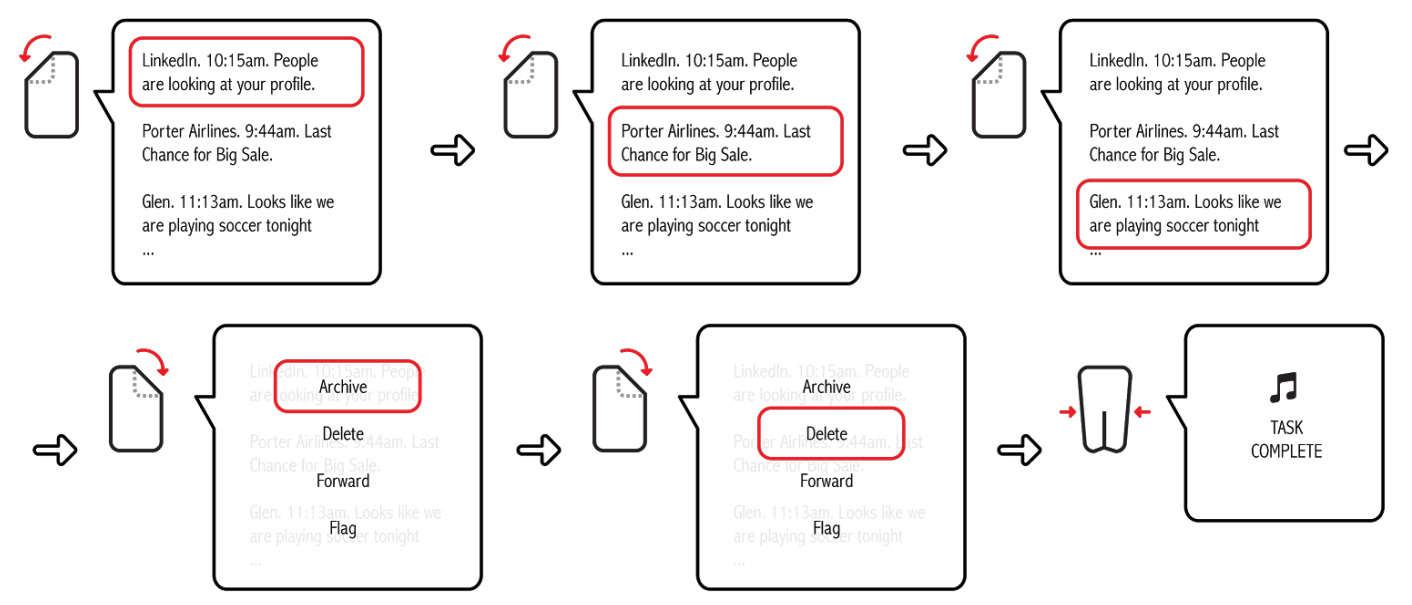

Figure 25 Typical user flow for participants completing an action task for deleting an email.

Based on observations, we noticed that 7 participants paused to re-position their hand(s) from a navigation position to perform the action switching, and 5 switched grip bending the top part of the device up and down. No participants needed to re-position their hands during navigation, which lead to the reduced differential.

5 participants re-gripped at some point during their study when performing the same repeated bend gesture to complete a task, and did so to perform a more aggressive and defined bend. This attempt to problem solve occurred when they did not receive a response from the system after several gesture attempts due to either an improper bend or the prototype not reading the sensor values correctly.

Re-gripping also occurred when participants needed to complete a task by squeezing the device. 7 participants re-gripped from the navigation, action or rotor position to the selection position to complete the task.

In comparison, participants did not need to re-position to complete the tasks nearly as much for touch interactions. The majority of participants switched from swiping horizontally to vertically with ease. All participants who held the touch prototype in one 
hand needed to re-position to complete the rotor-setting task as the gesture required a counter-clockwise motion with two fingers. This re-position just required moving the thumb out of the way and bringing the second hand into position with relatively quick completion times.

\subsubsection{Participants Grip}

Overall, participants held the prototype in different positions from single hand to two, across bend and touch, Figure 26 illustrates some of the different positions. $41 \%$ of participants used one hand to hold the bendable prototype while 59\% used two hands. $65 \%$ of users held the touch prototype with one hand and interacted using the thumb and $35 \%$ held it in one hand and used their index finger with the other. All participants except one used the same number of hands for both prototypes. We expected that all participants would hold the touch prototype in a similar manner throughout the tasks due to the familiarity to daily interactions with smartphones.
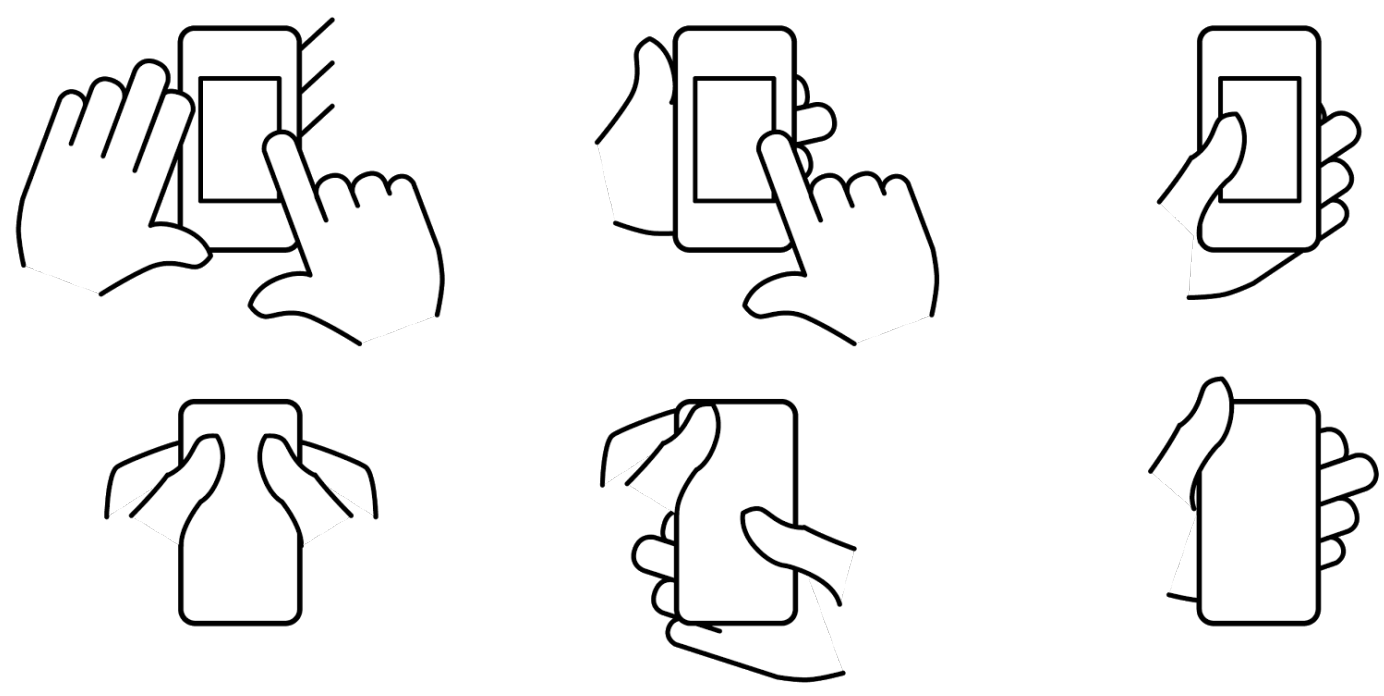

Figure 26 Illustrates some of the common hand positions participants used during the study. The top row illustrates touch hand grip and the bottom bend. 
Most participants did not identify physical effort, strain or phone size as a problem during the studies. Only 2 participants mentioned the device being too big and found the squeezing required effort. Both participants were female and had smaller hands.

\subsubsection{Bend and Touch Preference}

In a post study questionnaire, participants rated their comfort level using bend and touch gestures using a Likert Scale, both overall, and for each task set. (1: Not at all, 2: Not really, 3: Neutral, 4: Somewhat and 5: Very).

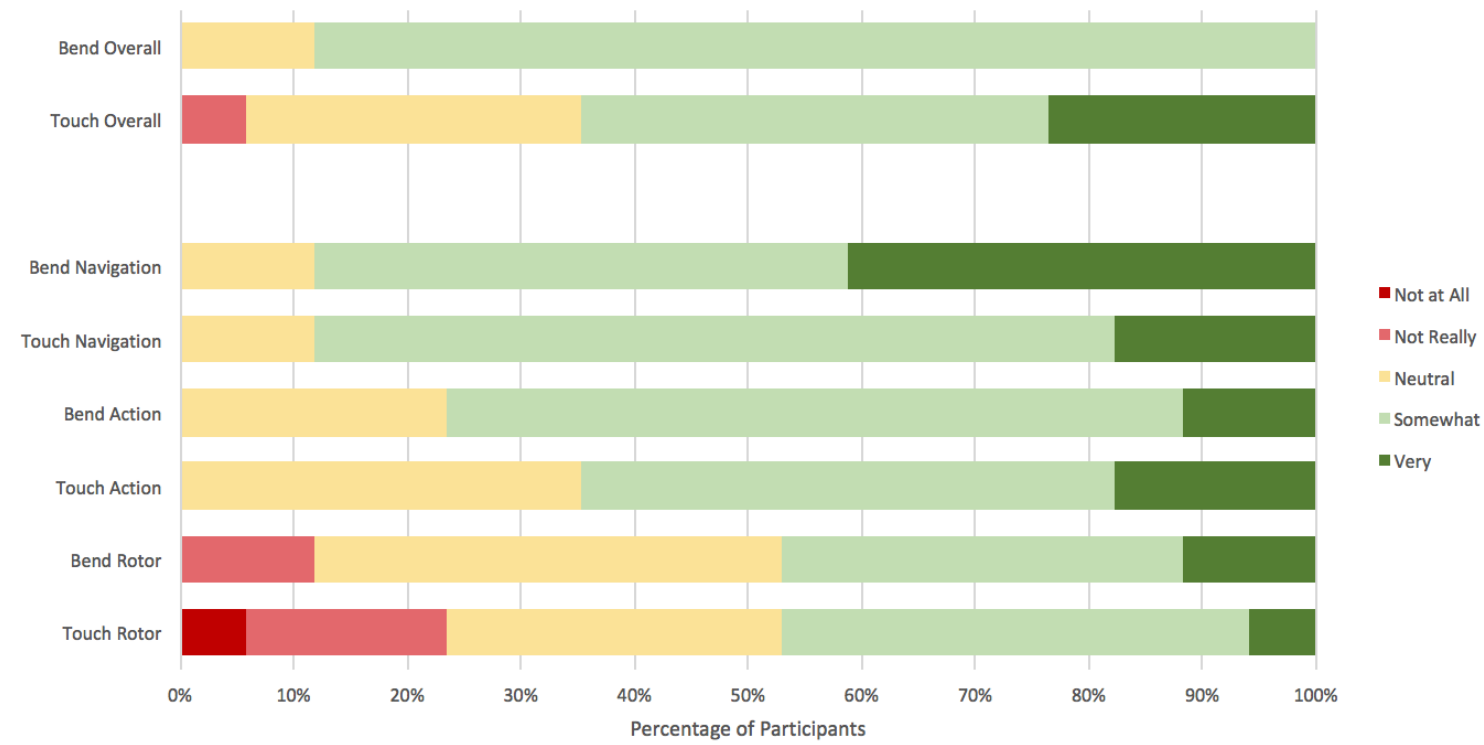

Figure 27 Distribution of Likert Scale responses for participants when asked to rate their comfort level when using bend and touch gestures.

We conducted a Wilcoxon signed-rank test for each of the responses comparing bend to touch (Figure 27). No comparison showed significant differences. Navigation produced a close to significant effect $(z=-1.941, p=.052)$, where $42 \%$ of participants 
found the bend gesture used to perform primary navigation very comfortable to interact with and only $17 \%$ for touch.

\subsubsection{Learnability of Bend and Touch Gesture Mapping}

In the post study questionnaire, participants rated their ease of learnability for the bend and touch gesture mapping using a Likert Scale. (1: Not at all, 2: Not really, 3: Neutral, 4: Somewhat and 5: Very). Figure 27 illustrates that although all participants had prior experience with touch gestures and none with bend gestures, they rated the learnability of both gesture mappings somewhat equally. $53 \%$ for touch and $58 \%$ for bend. After conducting a Wilcoxon signed-rank test for each of the responses comparing the learnability of bend to touch, we found no significant results.

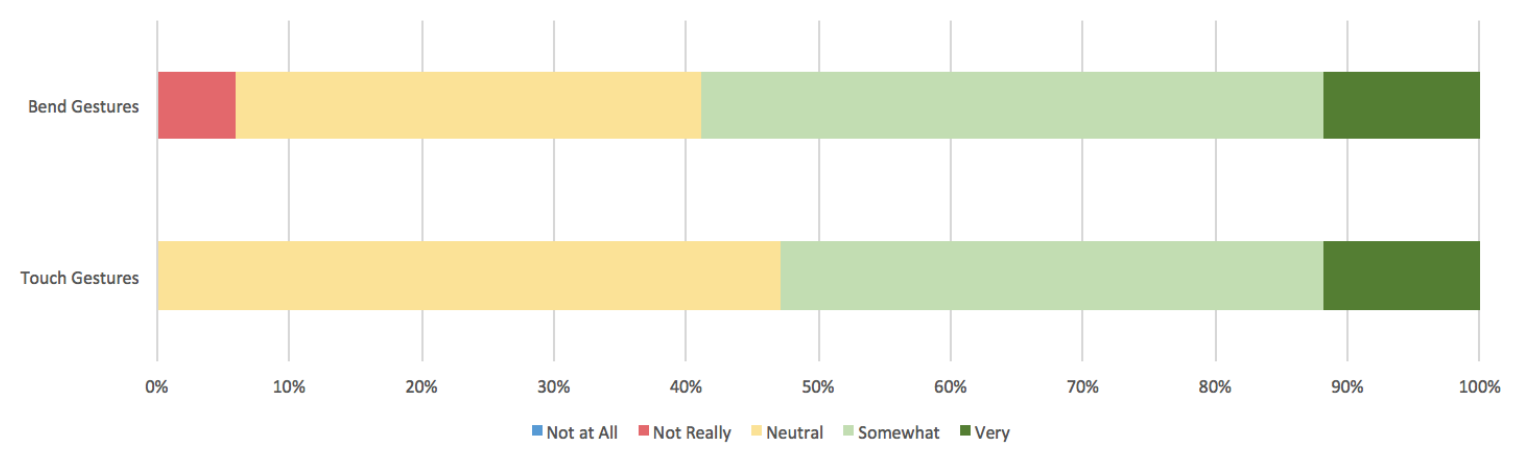

Figure 28 Distribution of Likert Scale responses for participants when asked to rate their ease of learnability when using bend and touch gestures.

\subsubsection{Visualizing the Interface}

Norman identifies that understanding the position of the user within a system is a fundamental usability heuristic [36] and applies to all types of users and interfaces. This becomes even more of a challenge for visually impaired users who cannot see the interface and must rely on memory and audio cues to understand the current system status. In the post study questionnaire, participants rated their confidence in 
understanding where in the system they were using a Likert Scale. (1: Not at all, 2: Not really, 3: Neutral, 4: Somewhat and 5: Very). After conducting a Wilcoxon signed-rank test for each of the responses comparing the participants understanding of the interface of bend to touch, we found no significant results.

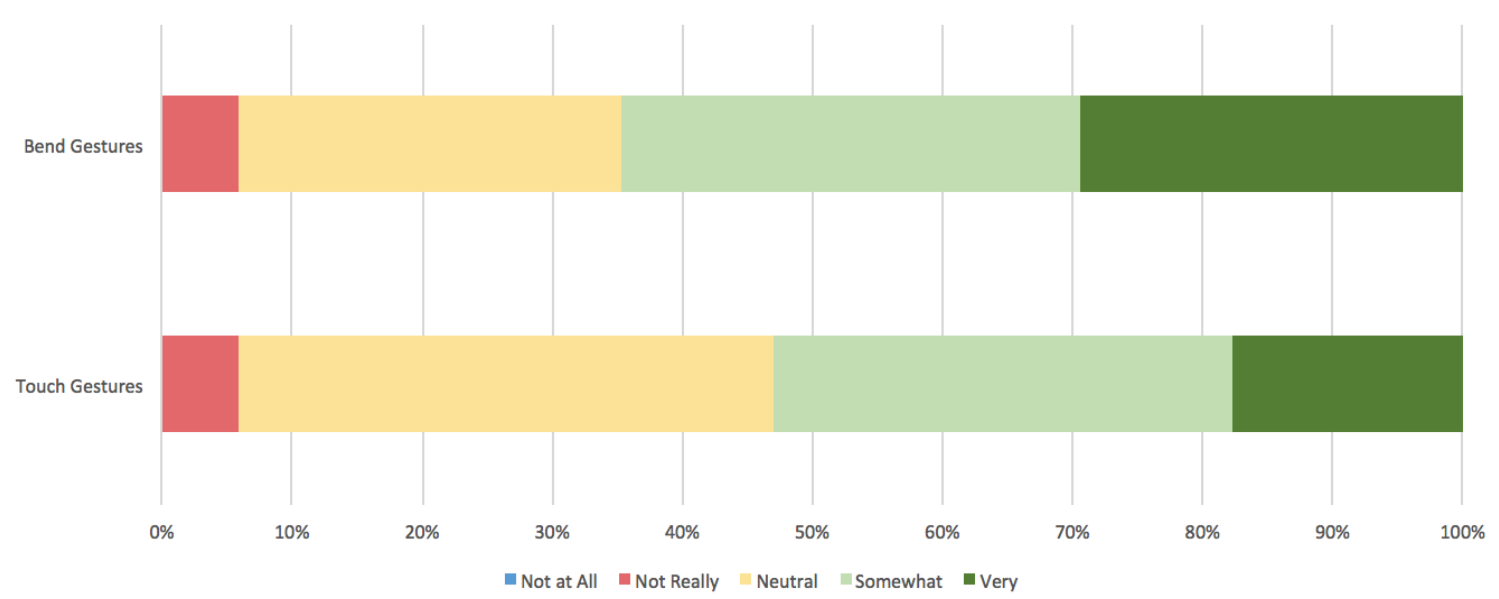

Figure 29 Distribution of Likert Scale responses for participants when asked to rate their understanding of focus placement when using bend and touch gestures.

\subsubsection{Qualitative Observations}

Throughout the experiment, we collected qualitative data through observation during the study and open-ended questions during the post study questionnaire. In addition to the observations regarding grip and re-positioning stated above, $59 \%$ of participants preferred using bend gestures to complete the tasks. Several participants commented on how easy bend gestures were to use and specifically the top corner for navigation. Several participants mentioned that bending up and down mapped better to the direction in the list they were navigating. 3 participants mentioned swiping left and right to navigate through a list as weird, and identified that touch gestures did not map as well as bend gestures to this direction of navigation. 4 participants who preferred touch 
made reference to the familiarity with the current interaction paradigms of today's smartphones.

Tactile feedback played an important role in this study as users were unable to see the device during the interactions. 7 people mentioned the preferable tactile nature of the bend gestures. 2 participants mentioned they actually used the groves on the back of the device as a reference for the corner locations. One participant identified that bend gestures were harder to confuse the different gestures where touch was too similar.

\subsection{Discussion}

We analyzed the data from the second study to gain insights into how users perform bend gestures in a visually impaired environment when compared to touch. We recorded completion times for each of the 9 tasks then averaged within each of the three task sets (navigation, action and rotor). Even though we did not identify any significant findings during task completion time, within the qualitative questioning, participants preferred the more tactile experience of using bend gestures overall but performed tasks slightly faster with touch. We interpret this to be a positive result, as it means that bend gestures were performed at a somewhat equivalent level to touch and were preferred by participants. With improvements to the prototype and an extended training period, we expect this time gap to be reduced or even reversed.

As mentioned, the slightly higher task completion times when using bend gestures could be attributed to participants re gripping the device during task completion, although further study could confirm this. Even though participants had an opportunity to utilize a trial period and become familiar with bend gestures, they did not have the same level of experience when compared to touch. Since all participants had prior experience with 
touch gestures and use a smartphone on a daily basis, their familiarity performing and switching between different gestures is much greater than compared to bend. This experience did not exist with bend gestures and could be attributed to the longer task completion times. Our original intent proposed using an iPhone sized device in portrait mode to reduce the need to re-position when completing these bend interactions, but most participants were unable to complete tasks without re-gripping.

Since screen size is not an issue for the visually impaired, the size of the device could be smaller for easier manipulation with one hand. Although only a couple of participants identified the device being too large, a smaller size could help participants switch from one gesture to the other. This could also help interactions with the top center gestures that were identified as more troublesome with participants.

The range in hand positions observed during the study identified that participants held the touch interface in a small set of familiar positions to that of a regular smartphone. In comparison, participants held the bend prototype in a range of positions that often changed throughout tasks. This was more evidence of their unfamiliarity with changing interactions and re-gripping on the fly potentially affecting the task completion time.

It is interesting to note that participants identified the more tactile nature of bend gestures when compared to touch. Due to the visual impairment imposed upon the participants, they were forced to rely on touch and audio feedback to successfully complete the tasks presented to them. Several identified that bend provided a more tactile form of interaction when interfacing with the system. The grooves on the back of the phone primarily created as stress points, unintentionally provided participants with an 
easy way to identify the different corner locations. Some participants identified the uniqueness between the different bend gesture locations and helped them differentiate between tasks and avoid confusion.

Learnability of interaction paradigms plays an important role for all users when confronted with new gesture patterns. In this study participants were given a unique set of gesture sets that mapped to interactions within a system, swiping left and right for example to navigate next and previous. Participants did not identify any significant difference between the learnability of bend or touch gestures, which contradicts our expectations that participants would rate the learnability for bend to be lower due to their unfamiliarity with the interaction paradigms.

These qualitative findings add additional value for bend gestures being a more tactile form of input. If a reduction in task completion time and re-gripping can be achieved with additional training and prototype refactoring this interaction technique could have validity as a primary form of interaction for the visually impaired. 


\section{Chapter: Discussion and Contributions}

We identify results in these studies that can help shape future application experiments of bend gestures for the visually impaired. Two key areas of application have been identified that could potentially increase the overall interaction experience for screen reader based smartphones. In a world where the visually impaired utilize their tactile sense to understand everything about them, an interaction paradigm that embodies a touch centric interaction approach could improve their overall experience and accessibility to technology.

During our second study, participants identified the superior tactile nature of interacting with bend gestures. Significant comments during the post study questionnaire lead us to conclude that participants found it easier to identify the different interactions they could perform. Several participants noted that the corners were easier to distinguish than the flat screen resulting in less confusion during interaction. For example, swiping up, down, left and right are less distinguishable than the top two corner bends. These findings reflect past work by Kane et al. [22] who studied touch gestures created by the visually impaired, and identified that they prefer multi touch concepts over single gestures to reduce the confusion between different actions. They also identified that blind participants favored corners or edges of touch screen devices, which directly supports the use of bend gestures that utilize these locations specifically.

The use of tactile affordances also surfaced when participants identified using the stress relief grooves on the back of the device as reference markers for bend gestures. Although not the original intent, these joints created tactile cue to users to understand the 
orientation and positioning of the device as well as a method to identify which bend gesture to perform.

In addition to the overall increased tactile experience, bend gestures show promise in improving the primary navigation method for these users. Interacting with VoiceOver, consists of navigating lists and systematically tabbing through any interface. When compared to touch, bend gestures were preferred by users for navigation and produced an almost equivalent task completion time. With improvements to the prototype sensitivity, smaller size and additional training, we are confident these times could be drastically reduced. The other locations: top center and top right although not as well liked and produced increased task times, could also improve with a smaller form factor and increased training.

The exploration of learnability and bend gesture prompts in the first study identified the corner closest to the thumb is a consistently reliable location for a haptic stimulus prompt. With almost a $92 \%$ accuracy rate among participants with a single motor Tacton, the user could not only interact with the interface but also receive haptic feedback and stimulus from the system. These notifications or prompts could be in the form of actionable alerts, notifications, or progress indicators. We aim to further explore and refine the design of Tactons for bend gesture prompts based on the findings from this study. Combining single and double motor Tactons to communicate layered information to the user, could allow a system to provide feedback in non-visual form.

The accuracy success of short single motor Tactons could expand on past work and be applicable within the context of visually impaired users. Azenkot et al. [2] explored the use of Tactons on a mobile phone for turn by turn way finding. 
This thesis has explored the two basic components of interaction design; input and feedback in the context of visual impairment and non-visual interaction design. The promising results found in the first study around the use of motor combination and intensity parameters in Tacton design, in conjunction with preference for certain bend gestures as a form of input lays the foundation for the overall design of a flexible smartphone designed specifically for the visually impaired.

With improvements to prototypes and continued exploration, the application of bend gestures for the visually impaired has validity as a primary source of input. From an implementation standpoint, several prototype and commercial smartphone devices exist for the visually impaired $[59,60]$, and we can envision a simple device that accepts bend interactions and outputs audio and tactile feedback as a competitive alternative. Eliminating the screen could drastically reduce the cost of this type of device and supply a simple, affordable and usable smartphone to over 285 million people. 


\section{Chapter: Conclusion}

The visually impaired face many challenges when interacting with technology on a daily basis. Their inability to visually process GUI based applications, or easily interact with the latest touch screen devices creates accessibility barrier to a wealth of information. This thesis explored the learnability and usability of bend gestures for the visually impaired and studied two critical components needed for these users to successfully learn and interact with any system.

\subsection{Research Overview}

Our first study explored the learnability of bend gestures through tactile means and identified if certain types of haptic stimulus could intuitively prompt a user to perform a certain bend gesture. We produced 48 Tactons consisting of a systematic combination of 5 parameters:

- Length: Short or Long Duration

- Wave: Sine or Square

- Intensity: Increasing or Decreasing

- Motors: Single or Double

- Location: Top Left, Top Center or Top Right

Our findings indicate that the motor parameter and the combination of motor and duration parameters produced significant accuracy across all three locations: top left, top center and top right. We also identified that intensity and motor combination produced significant agreement among participants on bend direction. Participants identified two 
motors to be less confusing in identifying bend direction than single motor. This relationship of vibration perception to direction needs further investigation with a realistic prototype that accepts bend gestures instead of an on screen interface.

Our second study compared the effectiveness of bend gestures as a primary form of input to touch, while receiving audio feedback in a device-occluded environment. We created 9 tasks across 3 task sets: Navigation, Action and Rotor. We analyzed the task completion times for each of the gesture and task sets, to determine if any significant difference existed between bend and touch.

Although touch slightly out performed bend across all three sets, the lack of significant difference could be attributed to participants re-gripping during task completion. With an extended training period and improved prototype, we are confident this completion time could be improved. To reduce the need to re-grip, we recommend further testing around the placement of the squeeze gesture closer to the top half of the phone. This would help reduce the need to re-grip to make a selection. Also, a smaller prototype would allow for more control and manipulation of the different corners.

In addition to quantitative data, participants rated their experience with interaction, learnability and interface visualization to provide a more holistic view of the experience. Although no significance between bend and touch existed for participants rating for visualization and understanding they did identify a significant preference for bend as the primary form of navigation.

\subsection{Contributions}

We contribute to the study of accessibility and DUIs for the visually impaired by exploring the application of bend gestures as a valid primary form of input for 
smartphone technology. Although further work needs to be completed, we identify significant findings for the use of a specific set of Tactons for learnability as well as evidence that bend gestures may be preferred for blind navigation interactions.

Our first objective explored if the use of Tactons could prompt bend gestures in the z-axis. We present a set of parameters used to design Tactons that can be further tested with an actual bendable prototype and visually impaired participants. To prompt location, a Tacton using a single motor and a short duration of 0.45 seconds resulted in an $86.89 \%$ accuracy rate. Wavelength or intensity had no significant impact on location choice. It is our recommendation to use shorter vibrations within localized Tactons to eliminate confusion and vibration spread to other parts of the device. In addition to location prompt, we recommend a double motor Tacton paired with intensity to prompt for bend direction. This could lead to future work combining multiple, short localized Tactons in succession, or the combination of short single motor and long double motor Tactons.

Our second objective aimed to identify if bend gestures would be a viable alternative to touch for the visually impaired, and, although we identify no significant data, the application looks promising. Our recommendation includes utilizing the corner closest to the thumb for any primary navigation tasks, but further prototype refinement and testing would need to be completed before recommendations for other gesture locations could be made.

\subsection{Limitations}

There were several limitations within both studies that could be improved in future work. Although the studies in this thesis used sighted participants with simulated 
vision loss, they were not ideal and a true representation of our specific demographic. It has been widely studied $[3,14,23]$ that the visual cortex of the visually impaired is used to process other senses such as hearing and touch. Although the degree of effectiveness is still un clear, it does attribute to their ability to rapidly read braille dots or listen to screen readers at over 25 syllables per second, well beyond a regular sighted individual at a mere six per second. This heightened sense of hearing and touch is not achievable with simulating visual impairment with a screen and an integral reason to test with this target group.

We plan to complete follow up studies with visually impaired users to confirm these findings. This conscious decision to test sighted users was made for several reasons. First they were more accessible during the testing phase, which allowed for flexibility during the testing procedure and reduced testing times overall. Second, due to the nature of the comparative second study, we strived to ensure an equal testing environment. We felt that participants with no prior experience in either VoiceOver or pre-defined mental models and experiences with non-visual based interactions would create consistent results. For the second study, we also focused on reaction time comparing gestures independent of their past experiences. Although qualitative data was captured from sighted individuals with respect to the overall experience, we aim to conduct further research with visually impaired participants to ensure this is truly the case. 5 studies identified in this thesis used visually impaired users $[12,21,22,30,39]$ and 3 used regular sighted $[31,38,49]$. For the most part, the studies that utilized blind participants studied usability concerns with existing software such as VoiceOver. The studies that used sighted participants conducted similar comparative or exploratory studies to ours. 
In addition to the lack of visually impaired participants, limitations within the first study primarily included the connection between the vibration motors and the participant's palm. Many trials attempted to embed motors into a silicone mold with no success and when Tactons were triggered they resonated throughout the prototype. Vertical alignment improved this slightly but in order to ensure the most effective delivery of vibration to the participant a thin cardboard sleeve was designed. Exploring how to embed stronger and more powerful motors into a silicone-based prototype that could be actually bent to perform the prompted gesture instead of entering it on screen would simulate a more realistic experience.

We also failed to eliminate the audible sound produced by the vibrations. The participant could still hear the motors even though the prototype remained out of sight. We are not sure if this had any bearing on the results as it still simulates a realistic environment, but future studies with participants wearing headphones could identify any significance.

The second study proved more challenging to complete, as both prototypes proved temperamental during the testing phase. To successfully test 17 participants, a total of 4 bendable prototypes were created. After a several rounds of testing, the bend sensors would no longer render values correctly and cause users to perform a bend and the gesture not register. This could have played a factor in the longer duration times for each task set. The touch prototype, although more reliable, could not replicate the sensitivity of a common smartphone and required users to slow their swiping to ensure an accurate reading. This sometimes resulted in a participant swiping but the system not 
responding. Although the majority of participants had no issue hearing the screen reader, two identified in the end survey that they struggled to distinguish some words.

\subsection{Future Work}

These findings can lead to future work in the fields of bend gestures, accessibility and haptic stimulus, building on our findings and other work in these respective fields. Future work could explore different grip patterns among participants or different device orientation. Our studies explored the flat, palm up orientation but other more common daily positions occur such as vertically or in the pocket that could change the perception of the participant's concept of up and down.

We also envision further exploration in the design of Tactons for alerts and prompts based on the guidelines outlined above or the combination of vibrations, such as a short single motor to identify location then a two motor stimulus to identify bend direction.

One of the big usability concerns identified by blind users is the inability to effectively input text. Although the second study primarily tested navigation, the exploration of bend gestures as text input for these users could be a viable alternative to other non visual keyboards. Future studies could explore these possibilities of solely using bend or a combination of bend and touch gestures as a source of input. This combination of touch and bend could extend beyond just text entry. Exploring the possibility of bending parts of the device to activate different modes that accept touch input.

A large area of further exploration could explore the improvement in prototypes used for future studies. Creating smaller prototypes to allow for easier gesture switching 
could help interacting with the top center gestures, identified as troublesome with participants. Using a bendable prototype with embedded motors, and testing different vibration motors types could improve the overall experience. One motor in particular worthy of further testing would be Piezo haptic motors. These motors are not bound by any constraints in amplitude as seen in the motors used in the first study. Piezo motors provide a much more localized and refined vibration when embedded into materials. We did not utilize these motors due to availability, cost and implementation complexity.

Other more exploratory work could further examine shape-changing devices for the visually impaired. For example, continuing work started by Lahey et al. with PaperPhone [27] would explore if the visually impaired could utilize a shape changing device to conduct different applications and change function. Other work by Tarun et al. [44] who explored shape changing devices in the form of notifications and system communication could also be expanded. We envision potential application for this type of work within the learnability of bend gestures. Imagine holding the device for the first time and the phone physically demonstrates how to conduct bend gesture with the top corner bending up. The visually impaired user would instantly know the basic interaction for that bend gesture in a very tactile manner.

Future work could explore the size and shape of the device for visually impaired participants. Since screen size and visual feedback is not a concern for this user population, conforming to a rectangle shape is no longer necessary. This opens the door to a variety of other shapes and sizes better suited for a more tactile experience, such as triangles, spheres, stars and many more. 
The work in this thesis leads to further research in this area with the ultimate goal of providing new technology and interaction patterns to improve the overall accessibility and usability of smartphone technology for the visually impaired. We look forward to continuing research in this area and improving and iterating on the findings identified in this paper. 


\section{Appendices}

\section{Appendix A Full list of Tacton designs}

\begin{tabular}{|c|c|c|c|c|c|c|}
\hline Tacton & Wave & Location Prompt & Intensity & Length & Pulse used & Motor number \\
\hline 10 & Square & Top Left & $\mathrm{Up}$ & Short & $50 \rightarrow 14$ & 2 \\
\hline 11 & Square & Top Left & Down & Short & $14 \rightarrow 50$ & 2 \\
\hline 12 & Square & Top Left & $\mathrm{Up}$ & Long & $67 \rightarrow 64$ & 2 \\
\hline 13 & Square & Top Left & Down & Long & $64 \rightarrow 67$ & 2 \\
\hline 14 & Square & Top Right & $\mathrm{Up}$ & Short & $50 \rightarrow 14$ & 3 \\
\hline 15 & Square & Top Right & Down & Short & $14 \rightarrow 50$ & 3 \\
\hline 16 & Square & Top Right & $\mathrm{Up}$ & Long & $67 \rightarrow 64$ & 3 \\
\hline 17 & Square & Top Right & Down & Long & $64 \rightarrow 67$ & 3 \\
\hline 18 & Square & Top & $\mathrm{Up}$ & Short & $50 \rightarrow 14$ & 4 \\
\hline 19 & Square & Top & Down & Short & $14 \rightarrow 50$ & 4 \\
\hline 20 & Square & Top & Up & Long & $67 \rightarrow 64$ & 4 \\
\hline 21 & Square & Top & Down & Long & $64 \rightarrow 67$ & 4 \\
\hline 22 & Sine & Top Left & $\mathrm{Up}$ & Short & 84 & 2 \\
\hline 23 & Sine & Top Left & Down & Short & 72 & 2 \\
\hline 24 & Sine & Top Left & $\mathrm{Up}$ & Long & 82 & 2 \\
\hline 25 & Sine & Top Left & Down & Long & 70 & 2 \\
\hline 26 & Sine & Top Right & $\mathrm{Up}$ & Short & 84 & 3 \\
\hline 27 & Sine & Top Right & Down & Short & 72 & 3 \\
\hline 28 & Sine & Top Right & $\mathrm{Up}$ & Long & 82 & 3 \\
\hline 29 & Sine & Top Right & Down & Long & 70 & 3 \\
\hline 30 & Sine & Top & Up & Short & 84 & 4 \\
\hline 31 & Sine & Top & Down & Short & 72 & 4 \\
\hline 32 & Sine & Top & Up & Long & 82 & 4 \\
\hline 33 & Sine & Top & Down & Long & 70 & 4 \\
\hline
\end{tabular}




\begin{tabular}{|c|c|c|c|c|c|c|}
\hline 34 & Sine & Top Left & Up & Short & $72 \rightarrow 84$ & $2 \rightarrow 1$ \\
\hline 35 & Sine & Top Left & Down & Short & $72 \rightarrow 84$ & $1 \rightarrow 2$ \\
\hline 36 & Sine & Top Left & $U p$ & Long & $70 \rightarrow 82$ & $2 \rightarrow 1$ \\
\hline 37 & Sine & Top Left & Down & Long & $70 \rightarrow 82$ & $1 \rightarrow 2$ \\
\hline 38 & Sine & Top Right & Up & Short & $72 \rightarrow 84$ & $3 \rightarrow 1$ \\
\hline 39 & Sine & Top Right & Down & Short & $72 \rightarrow 84$ & $1 \rightarrow 3$ \\
\hline 40 & Sine & Top Right & $\mathrm{Up}$ & Long & $70 \rightarrow 82$ & $3 \rightarrow 1$ \\
\hline 41 & Sine & Top Right & Down & Long & $70 \rightarrow 82$ & $1 \rightarrow 3$ \\
\hline 42 & Sine & Top & $U p$ & Short & $72 \rightarrow 84$ & $4 \rightarrow 1$ \\
\hline 43 & Sine & Top & Down & Short & $72 \rightarrow 84$ & $1 \rightarrow 4$ \\
\hline 44 & Sine & Top & $\mathrm{Up}$ & Long & $70 \rightarrow 82$ & $4 \rightarrow 1$ \\
\hline 45 & Sine & Top & Down & Long & $70 \rightarrow 82$ & $1 \rightarrow 4$ \\
\hline 46 & Square & Top Left & $\mathrm{Up}$ & Short & $50 \rightarrow 14$ & $2 \rightarrow 1$ \\
\hline 47 & Square & Top Left & Down & Short & $14 \rightarrow 50$ & $1 \rightarrow 2$ \\
\hline 48 & Square & Top Left & $\mathrm{Up}$ & Long & $67 \rightarrow 64$ & $2 \rightarrow 1$ \\
\hline 49 & Square & Top Left & Down & Long & $64 \rightarrow 67$ & $1 \rightarrow 2$ \\
\hline 50 & Square & Top Right & Up & Short & $50 \rightarrow 14$ & $3 \rightarrow 1$ \\
\hline 51 & Square & Top Right & Down & Short & $14 \rightarrow 50$ & $1 \rightarrow 3$ \\
\hline 52 & Square & Top Right & $\mathrm{Up}$ & Long & $67 \rightarrow 64$ & $3 \rightarrow 1$ \\
\hline 53 & Square & Top Right & Down & Long & $64 \rightarrow 67$ & $1 \rightarrow 3$ \\
\hline 54 & Square & Top & Up & Short & $50 \rightarrow 14$ & $4 \rightarrow 1$ \\
\hline 55 & Square & Top & Down & Short & $14 \rightarrow 50$ & $1 \rightarrow 4$ \\
\hline 56 & Square & Top & $\mathrm{Up}$ & Long & $67 \rightarrow 64$ & $4 \rightarrow 1$ \\
\hline 57 & Square & Top & Down & Long & $64 \rightarrow 67$ & $1 \rightarrow 4$ \\
\hline
\end{tabular}

Table 6 Outline of all possible Tactons presented to the user. Pulse number refers to the Tacton ID number referenced in the pre-packaged software [56]. Motor 1 was bottom, Motor 2 was top left, Motor 3 was top right and motor 4 was top center. 


\section{Appendix B Study 2 Task List}

\section{Find and Play (Task \#1)}

This task requires you to find a song and start playing it. You will navigate up and down through a list of songs and select "Sailors Eyes". When you are ready to start, squeeze the device as if you are launching the Music App. For simulation purposes in this browser - use the "x" key

\section{Find and Play (Task \#2)}

This task requires you to find a song and start playing it. You will navigate up and down through a list of songs and select "Wishful Thinking". When you are ready to start, squeeze the device as if you are launching the Music App. For simulation purposes in this browser - use the "x" key

\section{Find and Play (Task \#3)}

This task requires you to find a song and start playing it. You will navigate up and down through a list of songs and select "Cut Your Hair". When you are ready to start, squeeze the device as if you are launching the Music App. For simulation purposes in this browser - use the "x" key

\section{Email (Task \#4)}

This task requires you to find and forward an email from Ticket Master to your friend. You will navigate through your inbox and find the email from TicketMaster. When you reach that email use the secondary interaction to navigate between options and select 
"Forward". When you are ready, squeeze the device as if you are launching the Email App. For simulation purposes in this browser - use the " $\mathrm{x}$ " key

\section{Email (Task \#5)}

This task requires you to find and flag an email you recently received from Porter. You will navigate through your inbox and find the email from Porter. When you reach that email use the secondary interaction to navigate between options and select "flag". When you are ready, squeeze the device as if you are launching the Email App.

\section{Email (Task \#6)}

This task requires you to find the email from Paypal and move it to your junk folder.You will navigate through your inbox and find the email from PayPal. When you reach that email use the secondary interaction to navigate between options and select "move to junk". When you are ready, squeeze the device as if you are launching the Email App. For simulation purposes in this browser - use the "x" key

\section{Website Rotator Switch (Task \#7)}

This task requires to you change the VoiceOver Rotor Settings. Once it loads switch the rotator setting from "heading" to "links". When you are ready, squeeze the device as if you are opening the web page. For simulation purposes in this browser - use the "x" key 


\section{Maps Rotator Switch (Task \#8)}

This task requires to you change the VoiceOver Rotor Settings. Once the map app loads switch the rotator setting from "headings" to "speech rate". When you are ready, squeeze the device as if you are opening a page. For simulation purposes in this browser use the "x" key

\section{To-Dos Rotator Switch (Task \#9)}

This task requires to you change the VoiceOver Rotor Settings. Once the to-do app loads switch the rotator setting from "characters" to "sections". When you are ready, squeeze the device as if you are opening the to-do app. For simulation purposes in this browser - use the "x" key 


\section{Appendix C Study 2 End Questionnaire}

\begin{tabular}{|c|c|}
\hline $\begin{array}{l}\text { Overall, how comfortable were you, interacting with VoiceOver } \\
\text { using Bend Gestures? }\end{array}$ & $\begin{array}{l}1 \text { - Not at all } 2 \text { - Not really } \\
3 \text { - Neutral } 4 \text { - Somewhat } \\
5 \text { - Very }\end{array}$ \\
\hline $\begin{array}{l}\text { How quickly did you feel you learn't Bend gestures to interact } \\
\text { with VoiceOver? }\end{array}$ & $\begin{array}{l}1 \text { - Not at all } 2 \text { - Not really } \\
3 \text { - Neutral } 4 \text { - Somewhat } \\
5 \text { - Very }\end{array}$ \\
\hline $\begin{array}{l}\text { When completing the primary navigation tasks. (Eg. Navigating } \\
\text { up and down a list) how comfortable were you interacting with } \\
\text { VoiceOver using Bend Gestures? }\end{array}$ & $\begin{array}{l}1 \text { - Not at all } 2 \text { - Not really } \\
3 \text { - Neutral } 4 \text { - Somewhat } \\
5 \text { - Very }\end{array}$ \\
\hline $\begin{array}{l}\text { When completing the secondary action tasks. (Eg. switching } \\
\text { between different actions in the email list) how comfortable were } \\
\text { you interacting with VoiceOver using Bend Gestures? }\end{array}$ & $\begin{array}{l}\text { 1-Not at all } 2 \text { - Not really } \\
3 \text { - Neutral } 4 \text { - Somewhat } \\
5 \text { - Very }\end{array}$ \\
\hline $\begin{array}{l}\text { When completing the rotator setting tasks. (Eg. switching } \\
\text { between speech rate and headings) how comfortable were you } \\
\text { interacting with VoiceOver using Bend Gestures? }\end{array}$ & $\begin{array}{l}1 \text { - Not at all } 2 \text { - Not really } \\
3 \text { - Neutral } 4 \text { - Somewhat } \\
5 \text { - Very }\end{array}$ \\
\hline $\begin{array}{l}\text { Because you couldn't see the screen how confident were you in } \\
\text { understanding where in the interface you were when using bend } \\
\text { gestures? }\end{array}$ & $\begin{array}{l}1 \text { - Not at all } 2 \text { - Not really } \\
3 \text { - Neutral } 4 \text { - Somewhat } \\
5 \text { - Very }\end{array}$ \\
\hline Was there anything that you liked or didn't like about interacting & Text Entry \\
\hline
\end{tabular}


with bend gestures and receiving audio feedback?

Any other notes or observations?

Text Entry

Table End questionnaire completed by each participant following the section. 


\section{References}

1. Shiri Azenkot, Richard E Ladner, and Jacob O Wobbrock. 2011. Smartphone haptic feedback for nonvisual wayfinding. The proceedings of the 13th international ACM SIGACCESS conference on Computers and accessibility ASSETS '11, ACM Press, 281-282. http://doi.org/10.1145/2049536.2049607

2. Shiri Azenkot, Nicole B. Lee, Exploring the use of speech input by blind people on mobile devices, Proceedings of the 15th International ACM SIGACCESS Conference on Computers and Accessibility, p.1-8, October 21-23, 2013, Bellevue, Washington [doi>10.1145/2513383.2513440]

3. Marina Bedny, Alvaro Pascual-Leone, David Dodell-Feder, Evelina Fedorenko, and Rebecca Saxe. 2011. Language processing in the occipital cortex of congenitally blind adults. Proceedings of the National Academy of Sciences of the United States of America 108, 11: 4429-4434.

http://doi.org/10.1073/pnas.1014818108

4. S.A. Brewster and L.M. Brown. 2004. Tactons: structured tactile messages for non-visual information display. Proceeding AUIC '04 Proceedings of the fifth conference on Australasian user interface - Volume 28, 15-23. Retrieved from http://crpit.com/Vol28.html

5. $\quad$ Frederick P. Brooks, Ming Ouh-Young, James J. Batter, and P. Jerome Kilpatrick. 1990. Project GROPE Haptic displays for scientific visualization. ACM SIGGRAPH Computer Graphics 24, 4: 177-185.

http://doi.org/10.1145/97880.97899

6. Lorna M. Brown, Stephen A. Brewster, and Helen C. Purchase. 2006. Multidimensional tactons for non-visual information presentation in mobile devices. Proceedings of the 8th conference on Human-computer interaction with mobile devices and services - MobileHCI '06, ACM Press, 231-238. http://doi.org/10.1145/1152215.1152265

7. Lorna M. Brown, Stephen A. Brewster, and Helen C. Purchase. 2006. Tactile crescendos and sforzandos. CHI 'O6 extended abstracts on Human factors in computing systems - CHI EA '06, ACM Press, 610-615. http://doi.org/10.1145/1125451.1125578

8. Maria Claudia Buzzi, Marina Buzzi, Francesco Donini, Barbara Leporini, and Maria Teresa Paratore. 2013. Haptic reference cues to support the exploration of touchscreen mobile devices by blind users. Proceedings of the Biannual Conference of the Italian Chapter of SIGCHI on - CHItaly '13: 1-8. http://doi.org/10.1145/2499149.2499156 
9. Panteleimon Dimitriadis and Jason Alexander. 2014. Evaluating the effectiveness of physical shape-change for in-pocket mobile device notifications. Proceedings of the 32nd annual ACM conference on Human factors in computing systems - CHI '14: 2589-2592. http://doi.org/10.1145/2556288.2557164

10. Brian Frey, Caleb Southern, and Mario Romero. 2011. BrailleTouch: Mobile texting for the visually impaired. Lecture Notes in Computer Science (including subseries Lecture Notes in Artificial Intelligence and Lecture Notes in Bioinformatics) 6767 LNCS, PART 3: 19-25. http://doi.org/10.1007/978-3-64221666-4_3

11. DT Gallant, AG Seniuk, and Roel Vertegaal. 2008. Towards more paper-like input: flexible input devices for foldable interaction styles. Proc. UIST, 283-286. Retrieved May 25, 2012 from http://dl.acm.org/citation.cfm?id=1449762

12. Giuseppe Ghiani, Barbara Leporini and Fabio Paterno. 2008. Vibrotactile feedback as an orientation aid for blind users of mobile guides. MobileHCI '08: Proceedings of the 10th international conference on Human computer interaction with mobile devices and services: 431-434.

13. Audrey Girouard, Jessica Lo, Md Riyadh, Farshad Daliri, Alexander Keith Eady, and Jerome Pasquero. 2015. One-Handed Bend Interactions with Deformable Smartphones. Proceedings of the 33rd Annual ACM Conference on Human Factors in Computing Systems - CHI'15, ACM Press, 1509-1518. http://doi.org/10.1145/2702123.2702513

14. Frédéric Gougoux, Robert J. Zatorre, Maryse Lassonde, Patrice Voss, and Franco Lepore. 2005. A functional neuroimaging study of sound localization: Visual cortex activity predicts performance in early-blind individuals. PLoS Biology 3, 2: 0324-0333. http://doi.org/10.1371/journal.pbio.0030027

15. Gero Herkenrath, T. Karrer, and Jan Borchers. 2008. Twend: Twisting and Bending as new Interaction Gesture in Mobile Devices. Proc. CHI: 3819-3824.

16. Eve Hoggan, Roope Raisamo, and Stephen A. Brewster. 2009. Mapping information to audio and tactile icons. Proceedings of the 2009 international conference on Multimodal interfaces - ICMI-MLMI '09, ACM Press, 327. http://doi.org/10.1145/1647314.1647382

17. David Holman, Roel Vertegaal, Mark Altosaar, Nikolaus Troje, and Derek Johns. 2005. PaperWindows : Interaction Techniques for Digital Paper. Proc. CHI, 591599. http://doi.org/http://doi.acm.org/10.1145/1054972.1055054

18. Jeff Johnson, Teresa L. Roberts, William Verplank, et al. 1989. Xerox Star: A retrospective. Computer 22, 9: 11-26, 28. http://doi.org/10.1109/2.35211 
19. Tsien Tsuen-Hsuin Joseph Needham. 1985. Paper and Printing. Science and Civilisation in China: Volume 5, Part 1: 93 -96.

20. T. Kaaresoja and J. Linjama. 2005. Perception of short tactile pulses generated by a vibration motor in a mobile phone. First Joint Eurohaptics Conference and Symposium on Haptic Interfaces for Virtual Environment and Teleoperator Systems. World Haptics Conference: 2-3. http://doi.org/10.1109/WHC.2005.103

21. Shaun K Kane, Jeffrey P Bigham, and Jacob O Wobbrock. 2008. Slide rule. Proceedings of the 10th international ACM SIGACCESS conference on Computers and accessibility - Assets '08: 73-81. http://doi.org/10.1145/1414471.1414487

22. Sk Kane, Jo Wobbrock, and Re Ladner. 2011. Usable gestures for blind people: understanding preference and performance. Proceedings of the 2011 Conference on Human factors in computing systems - CHI'11: 413-422. http://doi.org/10.1145/1978942.1979001

23. C. M. Karns, M. W. Dow, and H. J. Neville. 2012. Altered Cross-Modal Processing in the Primary Auditory Cortex of Congenitally Deaf Adults: A VisualSomatosensory fMRI Study with a Double-Flash Illusion. Journal of Neuroscience 32, 28: 9626-9638. http://doi.org/10.1523/JNEUROSCI.6488-11.2012

24. Johan Kildal, Andrés Lucero, and Marion Boberg. 2013. Twisting Touch : Combining Deformation and Touch as Input within the Same Interaction Cycle on Handheld Devices. Proc. MobileHCI, 237-246. Retrieved January 27, 2014 from http://dl.acm.org/citation.cfm?id=2493238

25. Johan Kildal, Susanna Paasovaara, and Viljakaisa Aaltonen. 2012. Kinetic Device : Designing Interactions with a Deformable Mobile Interface. Proc. CHI EA, 18711876.

26. Johan Kildal. 2012. Interacting with deformable user interfaces: effect of material stiffness and type of deformation gesture. Haptic and Audio Interaction Design: $71-80$.

27. Byron Lahey, Audrey Girouard, Winslow Burleson, and Roel Vertegaal. 2011. PaperPhone: Understanding the Use of Bend Gestures in Mobile Devices with Flexible Electronic Paper Displays. Proc. CHI, 1303-1312. http://doi.org/10.1145/1978942.1979136

28. Sang-Su Lee, Sohyun Kim, Bipil Jin, et al. 2010. How Users Manipulate Deformable Displays as Input Devices. Proc. CHI: 1647-1656. http://doi.org/10.1145/1753326.1753572 
29. Sang-su Lee, Youn-kyung Lim, and Kun-Pyo Lee. 2012. Exploring the effects of size on deformable user interfaces. Proc. CHI, 89-93.

http://doi.org/10.1145/2371664.2371682

30. Barbara Leporini, Maria Claudia Buzzi, and Marina Buzzi. 2012. Interacting with mobile devices via VoiceOver. Proceedings of the 24th Australian ComputerHuman Interaction Conference on - OzCHI '12: 339-348. http://doi.org/10.1145/2414536.2414591

31. Kevin a. Li, Patrick Baudisch, and Ken Hinckley. 2008. BlindSight: Eyes-Free Access to Mobile Phones. Proceedings of the SIGCHI Conference on Human Factors in Computing Systems - CHI '08: 1389-1398. http://doi.org/10.1145/1357054.1357273

32. Jessica Lo and Audrey Girouard. 2014. Fabricating Bendy: Design and Development of Deformable Prototypes. IEEE Pervasive Computing Special Issue on Fabrication and Printing 13, 3: 40-46. http://doi.org/10.1109/MPRV.2014.47

33. Joseph Luk, Jerome Pasquero, Shannon Little, Karon MacLean, Vincent Levesque, and Vincent Hayward. 2006. A role for haptics in mobile interaction: initial design using a handheld tactile display prototype. Proceedings of the SIGCHI conference on Human Factors in computing systems 06, Figure 1: 171-180. http://doi.org/10.1145/1124772.1124800

34. Karon E. MacLean. 2008. Haptic Interaction Design for Everyday Interfaces. Reviews of Human Factors and Ergonomics 4, 1: 149-194. http://doi.org/10.1518/155723408X342826

35. Margaret Minsky, Ouh-young Ming, Oliver Steele, Frederick P. Brooks, and Max Behensky. 1990. Feeling and seeing: issues in force display. ACM SIGGRAPH Computer Graphics 24, 2: 235-241. http://doi.org/10.1145/91394.91451

36. Donald a Norman. Affordance, Conventions and Design. 1999. Design: 38-42.

37. Ivan Poupyrev and Shigeaki Maruyama. 2003. Tactile interfaces for small touch screens. Proceedings of the 16th annual ACM symposium on User interface software and technology - UIST '03, 6-9. http://doi.org/10.1145/964696.964721

38. Huimin Qian, Ravi Kuber, and Andrew Sears. 2009. Towards identifying distinguishable tactons for use with mobile devices. Proceeding of the eleventh international ACM SIGACCESS conference on Computers and accessibility ASSETS 09, 257-258. http://doi.org/10.1145/1639642.1639703

39. Jussi Rantala, Roope Raisamo, Jani Lylykangas, et al. 2009. Methods for presenting braille characters on a mobile device with a touchscreen and tactile 
feedback. IEEE Transactions on Haptics 2, 1: 28-39. http://doi.org/10.1109/TOH.2009.3

40. Alireza Sahami, Paul Holleis, Albrecht Schmidt, and Jonna Häkkilä. 2008. Rich Tactile Output on Mobile Devices. In Lecture Notes in Computer Science (including subseries Lecture Notes in Artificial Intelligence and Lecture Notes in Bioinformatics). 210-221. http://doi.org/10.1007/978-3-540-89617-3_14

41. Carsten Schwesig, Ivan Poupyrev, and Eijiro Mori. 2004. Gummi: A Bendable Computer. Proc. CHI: 263-270. Retrieved August 15, 2010 from http://dl.acm.org/citation.cfm?id=985726

42. AJ Sellen and RHR Harper. 2003. The myth of the paperless office. $231 \mathrm{p}$. Retrieved May 28, 2013 from http://portal.acm.org/citation.cfm?id=778158

43. Jürgen Steimle, Andreas Jordt, and Pattie Maes. 2013. Flexpad : Highly Flexible Bending Interactions for Projected Handheld Displays. Proc. CHI: 237-256.

44. Aneesh P Tarun, Byron Lahey, Audrey Girouard, Winslow Burleson, and Roel Vertegaal. 2011. Snaplet: Using Body Shape to Inform Function in Mobile Flexible Display Devices. Proceedings of the 2011 annual conference extended abstracts on Human factors in computing systems - CHI EA '11: 329-334. http://doi.org/10.1145/1979742.1979701

45. Aneesh P Tarun, Peng Wang, Paul R Strohmeier, Audrey Girouard, Derek Reilly, and Roel Vertegaal. 2013. PaperTab: Tablets as Thin and Flexible as Paper. Proceedings of the 2011 annual conference extended abstracts on Human factors in computing systems - CHI EA '13: 3131-3134.

46. The International Agency for the Prevention of Blindness (IAPB). 2012. WHO Facts. Retrieved from http://www.iapb.org/vision-2020/global-facts

47. Kristen Warren, Jessica Lo, Vaibhav Vadgama, and Audrey Girouard. 2013. Bending the Rules: Bend Gesture Classification for Flexible Displays. Proc. CHI. Retrieved May 30, 2013 from http://dl.acm.org/citation.cfm?id=2470740

48. Jun-ichiro Watanabe, A. Mochizuki, and Y. Horry. 2008. Bookisheet: Bendable Device for Browsing Content Using the Metaphor of Leafing Through the Pages. Proc. UbiComp: 360-369. Retrieved February 14, 2011 from http://portal.acm.org/citation.cfm?id=1409635.1409684

49. Koji Yatani and Khai Nhut Truong. 2009. SemFeel: A User Interface with Semantic Tactile Feedback for Mobile Touch-screen Devices. Proceedings of the 22nd annual ACM symposium on User interface software and technology - UIST '09, ACM Press, 111-120. http://doi.org/10.1145/1622176.1622198 
50. Shengdong Zhao, Pierre Dragicevic, Mark Chignell, Ravin Balakrishnan, and Patrick Baudisch. 2007. Earpod: eyes-free menu selection using touch input and reactive audio feedback. CHI 07 Proceedings of the SIGCHI conference on Human factors in computing systems: 1395-1404.

http://doi.org/10.1145/1240624.1240836

51. Apple VoiceOver, 2015. Retrieved from

https://www.apple.com/ca/accessibility/osx/voiceover/

52. Richard Coyne. 1992. The Role of Metaphor in Understanding Computers in Design. ACADIA Conference Proceedings: 3-11.

53. TalkBack, 2015. Retrieved from

https://play.google.com/store/apps/details?id=com.google.android.marvin.talkback $\& \mathrm{hl}=\mathrm{en}$

54. Blind Square, 2015. Retrieved from http://blindsquare.com/

55. VO Starter, 2015. Retrieved from https://itunes.apple.com/us/app/vostarter/id586844936? $\mathrm{mt}=8$

56. adafruit, 2015. Retrieved from http://www .adafruit.com/

57. dfrobot, 2015. Retrieved from http://www.dfrobot.com/

58. Global Mobile Economy Report, 2015. Retrieved from http://www.gsmamobileeconomy.com/GSMA_Global_Mobile_Economy_Report_ 2015.pdf

59. Project Ray, 2015. Retrieved from http://www.project-ray.com/

60. Assistech, 2015. Retrieved from https://assistech.com/store/telephones-for-theblind-and-visually-impaired 